\title{
Macromammal and bird assemblages across the late Middle to Upper Palaeolithic transition in Italy: an extended zooarchaeological review
}

\author{
Matteo Romandini ${ }^{\mathrm{a}, \mathrm{b}, *, 1}$, Jacopo Crezzini ${ }^{\mathrm{c}, 1}$, Eugenio Bortolini ${ }^{\mathrm{a}, 1}$, Paolo Boscato ${ }^{\mathrm{c}}$, \\ Francesco Boschin $^{\mathrm{c}}$, Lisa Carrera ${ }^{\mathrm{d}}$, Nicola Nannini ${ }^{\mathrm{e}}$, Antonio Tagliacozzo ${ }^{\mathrm{f}}$, Gabriele Terlato ${ }^{\mathrm{b}}$, \\ Simona Arrighi ${ }^{a, c}$, Federica Badino ${ }^{a, g}$, Carla Figus ${ }^{a}$, Federico Lugli ${ }^{a}$, Giulia Marciani ${ }^{\mathrm{a}}$, \\ Gregorio Oxilia $^{\mathrm{a}}$, Adriana Moroni ${ }^{\mathrm{c}}$, Fabio Negrino ${ }^{\mathrm{h}}$, Marco Peresani ${ }^{\mathrm{b}}$, Julien Riel-Salvatore ${ }^{\mathrm{i}}$, \\ Annamaria Ronchitelli ${ }^{c}$, Enza Elena Spinapolice ${ }^{j}$, Stefano Benazzi ${ }^{a}$ \\ ${ }^{a}$ Università di Bologna, Dipartimento di Beni Culturali, Via degli Ariani 1, 48121, Ravenna, Italy \\ ${ }^{\mathrm{b}}$ Università di Ferrara, Dipartimento di Studi Umanistici, Sezione di Scienze Preistoriche e Antropologiche, Corso Ercole I d'Este 32, 44100, Ferrara, Italy \\ ${ }^{\mathrm{c}}$ Università di Siena, Dipartimento di Scienze Fisiche, della Terra e dell'Ambiente, U.R. Preistoria e Antropologia, Via Laterina 8, 53100, Siena, Italy \\ ${ }^{\mathrm{d}}$ Università di Bologna, Dipartimento di Scienze Biologiche, Geologiche e Ambientali, Via Zamboni 67, 40126, Bologna, Italy \\ ${ }^{\mathrm{e}} \mathrm{MuSe}$ - Museo delle Scienze, Corso del Lavoro e della Scienza 3, I-38123, Trento, Italy \\ ${ }^{\mathrm{f}}$ Service of Bioarchaeology, Museo delle Civiltà, Museo Preistorico Etnografico "Luigi Pigorini", Piazzale G. Marconi 14, I-00144, Rome, Italy \\ ${ }^{\mathrm{g}}$ C.N.R, Istituto di Geologia Ambientale e Geoingegneria, 20126, Milano, Italy \\ ${ }^{\mathrm{h}}$ Università di Genova, Dipartimento di Antichità, Filosofia, Storia, Via Balbi 2, 16126, Genova, Italy \\ ${ }^{\text {i } U n i v e r s i t e ́ ~ d e ~ M o n t r e ́ a l, ~ D e ́ p a r t e m e n t ~ d ' A n t h r o p o l o g i e, ~ 2900, ~ E d o u a r d ~ M o n t p e t i t ~ B l v d, ~ M o n t r e ́ a l, ~ Q C, ~ H 3 T ~ 1 J 4, ~ C a n a d a ~}$ \\ ${ }^{\mathrm{j} S}$ Sapienza University of Rome, Dipartimento di Scienze dell'Antichità, Piazzale Aldo Moro 5, 00185, Roma, Italy
}

\section{A R T I C L E I N F O}

\section{Keywords:}

Protoaurignacian

Uluzzian

Late mousterian

Zooarchaeology

Aoristic analysis

Italy

\begin{abstract}
A B S T R A C T
Evidence of human activities during the Middle to Upper Palaeolithic transition is well represented from rockshelters, caves and open-air sites across Italy. Over the past decade, both the revision of taphonomic processes affecting archaeological faunal assemblages and new zooarchaeological studies have allowed archaeologists to better understand subsistence strategies and cultural behaviors attributed to groups of Neandertal and modern humans living in the region.

This work presents the preliminary results of a 5-years research programme (ERC n. 724046 - SUCCESS) and offers a state-of-the-art synthesis of archaeological faunal assemblages including mammals and birds uncovered in Italy between 50 and 35 ky ago. The present data were recovered in primary Late Mousterian, Uluzzian, and Protoaurignacian stratigraphic contexts from Northern Italy (Grotta di Fumane, Riparo del Broion, Grotta Maggiore di San Bernardino, Grotta del Rio Secco, Riparo Bombrini), and Southern Italy (Grotta di Castelcivita, Grotta della Cala, Grotta del Cavallo, and Riparo l'Oscurusciuto). The available Number of Identified Specimens (NISP) is analysed through intra- and inter-site comparisons at a regional scale, while aoristic analysis is applied to the sequence documented at Grotta di Fumane. Results of qualitative comparisons suggest an increase in the number of hunted taxa since the end of the Middle Palaeolithic, and a marked change in ecological settings beginning with the Protoaurignacian, with a shift to lower temperatures and humidity. The distribution of carnivore remains and taphonomic analyses hint at a possible change in faunal exploitation and butchering processing between the Middle and Upper Palaeolithic. A preliminary comparison between bone frequencies and the distribution of burned bones poses interesting questions concerning the management of fire. Eventually, the combined use of relative taxonomic abundance and aoristic analysis explicitly addresses time averaging and
\end{abstract}

\footnotetext{
* Corresponding author. Università di Bologna, Dipartimento di Beni Culturali, Via degli Ariani 1, 48121, Ravenna, Italy.

E-mail addresses: matteo.romandini@unibo.it (M. Romandini), jacopocrezzini@gmail.com (J. Crezzini), eugenio.bortolini2@unibo.it (E. Bortolini), paolo.boscato@unisi.it (P. Boscato), fboschin@hotmail.com (F. Boschin), lisa.carrera3@unibo.it (L. Carrera), nicola.nannini@muse.it (N. Nannini), antonio.tagliacozzo@beniculturali.it (A. Tagliacozzo), gabriele.terlato@unife.it (G. Terlato), simona.arrighi@unibo.it (S. Arrighi), federica.badino@unibo.it (F. Badino), carla.figus3@unibo.it (C. Figus), federico.lugli6@unibo.it (F. Lugli), giulia.marciani@unibo.it (G. Marciani), gregorio.oxilia3@unibo.it (G. Oxilia), adriana.moroni@unisi.it (A. Moroni), fabio.negrino@unige.it (F. Negrino), marco.peresani@unife.it (M. Peresani), julien.riel-salvatore@umontreal.ca (J. Riel-Salvatore), annamaria.ronchitelli@unisi.it (A. Ronchitelli), enzaelena.spinapolice@uniroma1.it (E.E. Spinapolice), stefano.benazzi@unibo.it (S. Benazzi).

${ }^{1}$ these authors equally contributed to the present work.
} 
temporal uncertainty embedded in NISP counts and offers estimates of absolute change over time that can be used to support hypotheses emerging from taxon relative frequencies.

\section{Introduction}

Evidence for change in human behaviour and adaptive strategies linked to palaeoenvironmental change has been consistently documented for contexts dated to Marine Isotope Stage 3 (MIS 3: 60-30 ky BP) across Europe. The different subsistence strategies developed by Neandertals and modern humans in response to change in the underlying climatic conditions has been of particular interest in all transitional contexts of continental and Mediterranean Europe (among others: Bietti and Manzi, 1990-91; Guidi and Piperno, 1992; Stiner, 1994; Bietti and Grimaldi, 1996; Milliken, 1999-2000; Kuhn and Bietti, 2000; Mussi, 2001; Peresani, 2009, 2011; Moroni et al., 2013, 2019).

Investigations into hominin diets, specifically those of the Neandertals, ineluctably feed into debates that revolve around the presumed capabilities, or lack thereof, of these hominins in the exploitation of small game as a food resource (Stiner, 2001; Stiner and Munro, 2002, 2011; Hockett and Haws, 2005).

Nevertheless, multiple data have induced some authors to suggest that the exploitation of small animals has been important for human subsistence since ca. 250ka (Klein and Scott, 1986; Stiner, 2005; Romandini et al., 2018b; Morin et al., 2019).

The Italian Peninsula plays a pivotal role as it connects Alpine Europe to the centre of the Mediterranean, and it provides a privileged perspective on interaction and replacement of Neandertals by modern humans in a very diverse set of ecological and climatic regions (Benazzi et al., 2011; Higham et al., 2011; Peresani, 2011; Moroni et al., 2018; Villa et al., 2018; Peresani et al., 2016, 2019). All scholars agree for example on the role played by geographic barriers (Alps and Apennines) in segregating - from a climatic and ecological point of view - a western Mediterranean region form an eastern continental one, the latter affected by the cyclical emersion of the northern Adriatic platform (Sala, 1990; Sala and Marchetti, 2006). Such a diversity, however, made the reconstruction of past ecosystems, of the spatio-temporal distribution of resources, and of population-level subsistence strategies particularly difficult, especially in light of the intense glacial/interglacial cycles of the past 200,000 years. Notwithstanding the many detailed studies carried out at a local scale, a global understanding of change in mobility, adaptive strategies, and settlement pattern across the Middle-Upper Palaeolithic Transition across Italy is still elusive. The few exceptions (Van Andel and Davies, 2003) draw on very scant and heterogeneous data generated with different aims and at different scales, and the emerging scenarios are far from the temporal coherence exhibited by recent global (Bond et al., 1992; Dansgaard et al., 1993; Rasmussen et al., 2014) and Mediterranean palaeoclimatic and palaecological records (Allen et al., 1999; Sánchez Goñi et al., 2000; Tzedakis et al., 2002; Margari et al., 2009; Fletcher et al., 2010; Müller et al., 2011; Wulf et al., 2018).

The present paper aims to fill this gap and lay the foundations for a finer and more systematic comparison across the whole of the Italian Peninsula by presenting a state-of-the-art review of available data on faunal remains in a number of key dated Italian sites. By carefully documenting and comparing the distribution of faunal remains, we also generate hypotheses on the different subsistence strategies developed by Neandertals and modern humans in response to change in the underlying climatic conditions. Inferences about paleoclimate and ecological settings are based on well-established links between ungulate families/avifaunal groups and the very specific environmental settings to which they were and still are adapted today. More specifically, the review focuses on relative taxon frequency of macromammals (ungulates, carnivores, rodents and lagomorphs) and birds across Late
Mousterian, Uluzzian, and Protoaurignacian layers documented for 9 Italian sites for which quantitative data are available (Fig. 1, Areas 1-3). Other assemblages from central and northwestern Italy are also briefly described in this context, but their data are not directly integrated in more detailed investigations of regional trends. Finally, one particularly well-documented site (Grotta di Fumane) is also investigated through aoristic analysis, a probabilistic approach never before applied to the Palaeolithic of Italy. The method explicitly addresses temporal uncertainty and depositional factors affecting the observed number of specimens (NISP) and offers estimates of absolute change over time that can be used to support hypotheses emerging from taxon relative frequencies, as well as to allow a direct comparison between layers of different coeval sites. Finally, the available taphonomic evidence is also presented to provide preliminary insights on change over time in animal exploitation strategies and butchering processes.

This work is still preliminary, as it describes the initial results of an ongoing 5-year project aimed at reaching a deeper understanding of the mechanisms that underpinned the geographic overlap between Neandertals and modern humans in the Italian Peninsula, as well as the final replacement of the former by the latter. While future research will be able to support or disprove part of the picture that emerges from this first assessment, it nonetheless offers a first attempt to generate a coherent synthesis of all the data published to date concerning the region of interest.

\section{Regional contexts}

\subsection{Northeastern (Adriatic) Italy}

In the northern Adriatic Area archaeologists uncovered a considerable number of rock shelters and caves which yielded evidence of the last Neandertals and of the earliest modern humans. The geographic location of such sites is a key element to understanding regional differences in the faunal assemblages they have yielded (Sala, 1990; Sala and Marchetti, 2006; Sala and Masini, 2007; Masini and Sala, 2007, 2011). From a paleoecological point of view, pollen records from Lake Fimon and Azzano Decimo (north-eastern Alpine foothills; Pini et al., 2009, 2010), document long-term vegetation trends during MIS 3. Phases of expansion of conifer-dominated forest (Pinus sylvestris/mugo and Picea), rich in broad-leaved trees (Alnus cf. incana and tree Betula), are accompanied by a reduction in the amount of warm-temperate elements (e.g. Tilia). Middle Würm stadials experienced summer temperatures very close to the growth limit of oaks, but still within the range of lime (MAW $13-15{ }^{\circ} \mathrm{C}$ ) which persisted, together with other temperate trees (e.g. Abies), up to ca. 40 ka (Pini et al., 2009, 2010; Badino et al., this Special Issue). Interestingly, peaks of Tilia pollen have been identified in layers preserving Mousterian artifacts and dated to 40.6-46.4 ka ${ }^{14} \mathrm{C}$ BP from cave deposits at the Broion (Leonardi and Broglio, 1966; Cattani and Renault-Miskowski, 1983-84). Despite evidence of afforestation persisting at a long-term scale south of the Alps, forest withdrawals with expansion of grasslands and dry shrublands (Gramineae, Artemisia, Chenopodiaceae) occurred, possibly related to the establishment of drier/colder conditions (i.e. Greenland Stadials/ Heinrich events). Such drier and colder stadial conditions likely favoured the presence of Alpine ibex, chamois, and marmot at low altitudes (in the Colli Berici), as well as the presence of micromammals in steppic environments, and the diffusion of birds in tundra-like environments. The Po alluvial valley was, in contrast, inhabited by woolly rhinoceros, mammoth, and bison (Sala, 1990).

Only a few contexts offer data on the Middle to Upper Palaeolithic 
transition, and their number further decreases for the temporal interval comprised between 50 and $35 \mathrm{ky}$.

At present, data on faunal remains and the relative chronology are available from Grotta di Fumane (Verona), Grotta Maggiore di San Bernardino (Vicenza), Riparo del Broion and Grotta del Broion (Vicenza), Grotta del Rio Secco (Pordenone) (Table 1, Fig. 1, Area 1). Grotta di Fumane is a key site for northern Italy, located at $350 \mathrm{~m}$ asl in the western part of the Lessini Mountains (Table 1, Fig. 1). Its archaeological sequence includes the Middle-Upper Palaeolithic transition (Peresani et al., 2008; Higham et al., 2009; López-García et al., 2015). Faunal assemblages consist of a rich association of ungulates, carnivores, and birds from diverse environments and climates. Quantitative comparisons between the Uluzzian assemblage (A3) (Tagliacozzo et al., 2013) and the late Mousterian ones (A4, A5-A6, A9) has highlighted only modest ecological and economic adjustments within a humid forested landscape (Peresani et al., 2011a,b; Romandini, 2012; Romandini et al., 2014a, 2016a,b, 2018a,b, Fiore et al., 2016; Gala et al., 2018; Terlato et al., 2019). Considerable change, on the other hand, emerged from the Protoaurignacian occupations (A2), coinciding with a shift towards colder and steppic environments (Cassoli and Tagliacozzo, 1994a; Fiore et al., 2004).

Riparo del Broion is located in the northern part of the Berici eastern slope, at $135 \mathrm{~m}$ asl, along a steep slope comprising escarpments, cliffs and remnants of collapsed sinkholes that connects the top of Mount Brosimo (327 $\mathrm{m}$ asl) to the marshy and swampy plain (De Stefani et al., 2005; Gurioli et al., 2006; Romandini et al., 2012; Peresani et al., 2019).

Slope-waste clay deposits can be found at the feet of Mount Brosimo. Uluzzian faunal assemblages (levels $1 \mathrm{f}-1 \mathrm{~g}$ ) show a high richness due to the different environments of the surroundings. Alongside the presence of marmot, hare, chamois, ibex, bison and possibly aurochs, the number of red deer and roe deer bones as well as the abundance of wild boar remains indicate the existence of humid woodlands located in the alluvial plain to the east of Mount Brosimo (Peresani et al., 2019).

Grotta Maggiore di San Bernardino opens on the eastern slope of the Berici karst plateau $135 \mathrm{~m}$ asl, to the west of the alluvial plain of the Bacchiglione River. Eight lithological units compose a Middle-Late Pleistocene stratigraphic sequence (Cassoli and Tagliacozzo, 1994b; Peresani, 2001). The majority of the total faunal remains found at the

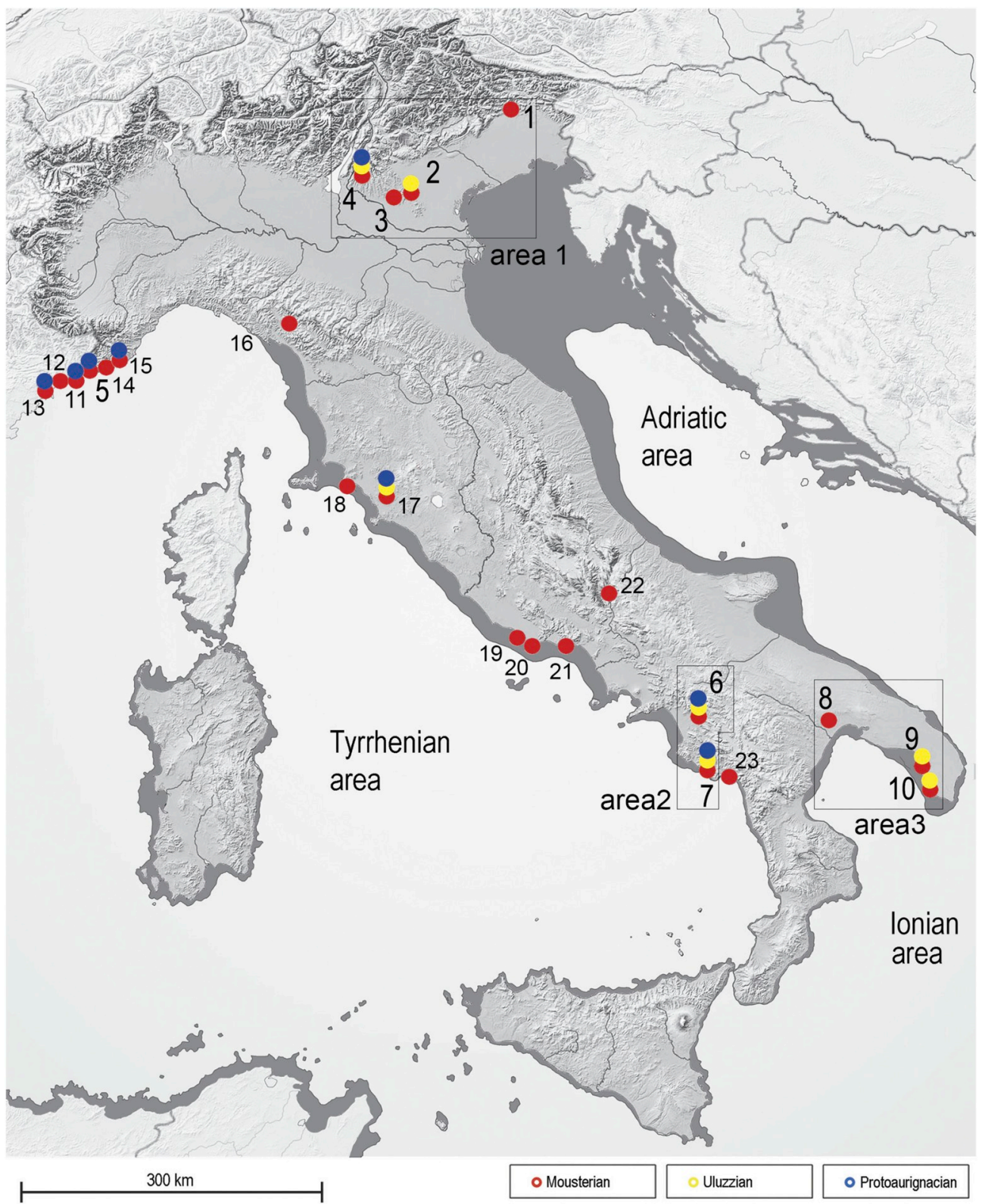

Fig. 1. MIS 3 map of Italy (modified from Moroni et al., 2018) and the geographic location of the sites with previously published faunal assemblages mentioned in this work and dated between ca. 50 and $38 \mathrm{ky}$ ago. Sites that are part of the project ERC n. 724046 - SUCCESS are numbered from 1 to 10. Sites analysed in this work are assigned numbers 1-9 (with the exclusion of 5), and are located in study Areas 1-3 (Northeastern, Southwester/Tyrrhenian, and Southeastern/Ionian respectively). For each site the colors represent the presence of levels, USS and/or layers chronologically and technologically linked respectively to the Protoaurignacian = blue; Uluzzian = yellow; Late Mousterian = red. 1) Grotta del Rio Secco; 2) Riparo del Broion; 3) Grotta di San Bernardino; 4) Grotta di Fumane; 5) Riparo Bombrini; 6) Grotta di Castelcivita; 7) Grotta della Cala; 8) Riparo l'Oscurusciuto; 9) Grotta del Cavallo; 10) Grotta di Uluzzo C; 11) Riparo Mochi; 12) Grotta del Principe; 13) Observatoire; 14) Arma Manie; 15) Arma degli Zerbi; 16) Buca della Iena; 17) Grotta la Fabbrica; 18) Grotta dei Santi; 19) Grotta Breuil; 20) Grotta del Fossellone; 21) Grotta S. Agostino; 22) Grotta Reali; 23) Riparo del Poggio. The Italian Peninsula shows a sea level of $70 \mathrm{~m}$ below the present-day coastline, based on the global sea-level curve (Benjamin et al., 2017) but lacking the estimation of post-MIS3 sedimentary thickness and eustatic magnitude (sketch map courtesy of S. Ricci, University of Siena). (For interpretation of color coding in this figure legend, the reader is referred to the Web version of this article.) 
site $(78 \%)$ belongs to ungulates, although the frequency of ungulate remains varies between stratigraphic units (Table 1). Units II + III, associated to late Mousterian frequentation, is the only layer to have undergone a detailed zooarchaeological study. Its assemblage suggests the presence of humid climatic conditions, the expansion of woodlands (Cassoli and Tagliacozzo, 1994b; Peresani, 2011; López-García et al., 2017; Romandini et al., 2018b; Terlato et al., 2019).

Grotta del Rio Secco is located in a stream gorge at $580 \mathrm{~m}$ asl on the Pradis Plateau in the eastern part of the Carnic Pre-Alps (Fig. 1 and Table 1), an orographic system dissected by N-S and W-E valleys separating mountains with peaks of $2000-2300 \mathrm{~m}$ asl. The site is a flat and wide south-facing shelter, with a gallery completely filled with sediments. The outer area of the shelter presents with a heap of large boulders collapsed from the original, larger roof. Human occupation has been dated (Tables 1 and A.1) to the Late Mousterian (layers 5top, 7, 5, and 8) and to the Gravettian (layers 6 and 4) (Peresani et al., 2014; Talamo et al., 2014). In layers 7 and 8 archaeologists found evidence of the use of fire and of an intensive exploitation of carnivores (Ursus arctos, Ursus spelaeus, mustelids, and canids), which are more numerous than ungulates (Peresani et al., 2014; Romandini et al., 2018a). Although bird remains are rare, a terminal pedal phalanx of a golden eagle with anthropic cut marks on the proximal articular facet was recovered from layer 7 (Romandini et al., 2014b).

\subsection{Northwestern (Tyrrhenian) Italy}

The coastal area of this region is characterized by a particular relief pattern with middle-range mountains and a narrow littoral plain between the Mediterranean Sea and the southern Alps (Fig. 1). Faunal assemblages of the region date to between Marine Isotope Stage (MIS) 6 and 3 , in agreement with geochronological, pollen and cultural data (Valensi and Psathi, 2004; Bertola et al., 2013; Romandini, 2017). From a general point of view, faunal assemblages attributed to the Middle to Upper Palaeolithic transition exhibit high taxonomical richness, reflecting a variety of biotopes such as forest hills, coastal plains, narrow valleys in the hinterland and numerous cliffs. Consistently high values in species richness, in particular for carnivores, were recorded in $\mathrm{Li}$ guria during MIS 3 and 2 (Valensi and Psathi, 2004). The most frequent species of ungulates and small mammals point to the extensive presence of forested environments.

A variety of Late Mousterian sites are reported (Fig. 1): Arma delle Manie, Grotta degli Zerbi, Riparo Bombrini, Riparo Mochi, Grotta del Principe in Italy, and Grotte de l'Observatoire in the Principality of Monaco. The arrival of modern humans in the region is associated to a marked change in the archaeological record (Negrino and RielSalvatore, 2018; Riel-Salvatore and Negrino, 2018a). At present, Protoaurignacian evidence has been uncovered at Riparo Mochi (Alhaique, 2000; Kuhn and Stiner, 1998; Douka et al., 2012; Grimaldi et al., 2014), Riparo Bombrini (Bertola et al., 2013; Holt et al., 2019; Negrino and Riel-Salvatore, 2018; Riel-Salvatore et al., 2013; Riel-Salvatore and Negrino, 2018a, 2018b), Arma degli Zerbi and Grotte de l'Observatoire (Rossoni-Notter et al., 2016; Onoratini, 2004; Onoratini and Simon, 2006; Porraz et al., 2010; Romandini, 2017).

\subsection{Southern Italy}

Palaeoecological data for southern Italy come from the Lago Grande di Monticchio record (Monte Vulture, Basilicata). During MIS 3, pollen

Table 1

Detailed context table of sites analysed in this work with reference to dominant taxa and to the most represented environmnetal setting recorded at each site.

\begin{tabular}{|c|c|c|c|c|c|c|c|c|}
\hline & Sites & US/levels & Technocomplex & $\mathrm{C} 14 \mathrm{cal} \mathrm{BP}$ & $\mathrm{U} / \mathrm{Th}$ & Tot. NISP Ungulates & Dominant taxa & Climate/Enviroment \\
\hline $\mathrm{N}$ & RS-Rio Secco & $5+8$ & Late Mousterian & $>48-44 \mathrm{ky}$ BP & - & 42 & Ursus sp. & cold-temperate climate with humid condition and open enviroments \\
\hline$\circ$ & RF-Fumane & A9 & Late Mousterian & 47-45 ky BP & - & 1214 & \multirow{3}{*}{ Cervus elaphuhs + Capreolus capreolus } & \multirow{3}{*}{ temperate climate with forests and clearings } \\
\hline $\mathrm{t}$ & RF-Fumane & A6 & Late Mousterian & \multirow{2}{*}{$44-42$ ky BP } & - & 1570 & & \\
\hline $\mathrm{h}$ & RF-Fumane & $A 5 / A 5+A 6$ & Late Mousterian & & - & 479 & & \\
\hline$\stackrel{e}{r}$ & RS-Rio Secco & 5top+7 & Late Mousterian & $>49-41$ ky BP & - & 58 & Ursus sp. & cold-temperate climate with humid condition and open enviroments \\
\hline$n$ & SB-S. Bernardino & $\|+111$ & Late Mousterian & & $35-54 \mathrm{ky}$ & 694 & Cervus elaphuhs + Capreolus capreolus & temperate climate with humid conditions and woodland covering \\
\hline $\mathrm{I}$ & RF-Fumane & A4 & Late Mousterian & 45-44 ky BP & - & 484 & Cervus elaphuhs + Capra ibex & cold-temperate climate with alpine setting and open environments \\
\hline $\mathrm{t}$ & RB-Broion & $1 \mathrm{e}+1 \mathrm{f}+1 \mathrm{~g}$ & Uluzzian & 38 ky BP & - & 59 & Sus scrofa & cold-temperate climate with humid woodlands \\
\hline i & RF-Fumane & A3 & Uluzzian & 44-42 ky BP & - & 452 & Cervus elaphuhs + Capra ibex & cold-temperate climate with alpine setting and open environments \\
\hline y & RF-Fumane & A2-A2R & Protoaurignacian & 40-34 ky BP & - & 795 & Capra ibex & cold climate with steppic enviroments \\
\hline \multirow{11}{*}{$\begin{array}{l}\mathrm{s} \\
\mathrm{o} \\
\mathrm{u} \\
\mathrm{t} \\
\mathrm{h} \\
\mathrm{e} \\
\mathrm{r} \\
\mathrm{n}\end{array}$} & Cala & $\mathrm{R}$ & Late Mousterian & - & - & - & - & - \\
\hline & CTC-Castelcivita & $32-21$ & Late Mousterian & \multirow{2}{*}{$46-42$ ky BP } & - & \multirow{2}{*}{453} & Dama dama + Cervus elaphus & temperate woodland covering \\
\hline & CTC-Castelcivita & 20-18lower & Late Mousterian & & - & & Rupicapra sp. + Cervus elaphus & woodland covering and increasing in humidity \\
\hline & T CTC-Castelcivita & 18upper-13 & Uluzzian & - & - & 134 & Capreolus capreolus + Rupicapra sp. & temperate climate with more dispersed woodlands \\
\hline & CTC-Castelcivita & $12-10$ & Uluzzian & $42-40.5$ ky BP & - & 110 & Equus ferus & cold climate and increased presence of open enviroments \\
\hline & Cala & 14 & Uluzzian & - & - & 347 & Dama dama & temperate climate and mediterranean evergreen \\
\hline & $i_{i}^{n}$ CTC-Castelcivita & 10upper-8 & Protoaurignacian & - & - & 33 & Equus ferus + Sus scrofa & cold climate with woodland covering and open enviroments \\
\hline & $\begin{array}{l}a_{n} \\
\text { CTC-Castelcivita }\end{array}$ & 7-top sequence & Protoaurignacian & - & - & 60 & Cervus elaphus + Rupicapra sp. & cold-temperate climate \\
\hline & Cala & 13 & Protoaurignacian & - & - & 230 & \multirow{3}{*}{ Cervus elaphus } & \multirow{3}{*}{ onset of cold climate with dispersal woodlands } \\
\hline & Cala & 12 & Protoaurignacian & - & - & 428 & & \\
\hline & Cala & $11-10$ & Protoaurignacian & - & - & 228 & & \\
\hline $\mathrm{t}$ & CAV-Cavallo & FIIIE & Late Mousterian & - & - & 349 & Bos primigenius + Cervus elaphus & open/forest steppe \\
\hline a & CAV-Cavallo & FIIIB-D & Late Mousterian & - & - & 268 & Dama dama + Bos primigenius & temperate phase \\
\hline \multirow[t]{6}{*}{ y } & CAV-Cavallo & FIIIA-FI & Late Mousterian & $>45 \mathrm{ky} \mathrm{BP}$ & - & 253 & Bos primigenius + Cervus elaphus & semi-arid stage/forest steppe \\
\hline & n. OSC-Oscurusciuto & $4-13$ & Late Mousterian & - & - & 574 & Bos primigenius & wooded meadows and open spaces \\
\hline & $\begin{array}{l}\text { A OSC-Oscurusciuto } \\
\text { d OS }\end{array}$ & 3 & Late Mousterian & - & - & 57 & Equus ferus + Bos primigenius & semi-arid stage/forest steppe \\
\hline & OsC-Oscurusciuto & 2-29-30-31 & Late Mousterian & - & - & 185 & Bos primigenius + Equus ferus & semi-arid stage/forest steppe \\
\hline & it OSC-Oscurusciuto & 1 & Late Mousterian & 43-42 ky BP & - & 40 & Bos primigenius + Cervus elaphus & temperate phase \\
\hline & CAV-Cavallo & EIII & Uluzzian & $45-43$ ky BP & - & 194 & Bos primigenius + Equus ferus & cold climate with more dispersed woodlands \\
\hline
\end{tabular}

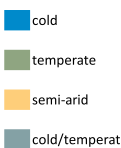


data associations indicate an alternation between cold/dry steppic vegetation (Artemisia-dominated steppe/wooded steppe), related to Greenland Stadials/Heinrich events (GSs/HEs), and an increased range of woody taxa including deciduous Quercus, Abies and Fagus (up to $30-60 \%$ of arboreal pollen), referred to Greenland Interstadials (GIs) with a maximum expansion between ca. $55-50$ ka (i.e. GI 14) (Allen et al., 1999; Fletcher et al., 2010; Badino et al., this Special Issue). Nevertheless, faunal assemblages coming from MIS3-aged stratigraphic sequences highlight different climatic trends between Tyrrhenian (southwestern) and Ionian (southeastern) contexts (Boscato, 2017) due to an almost persistent moisture availability on the former, mainly generated by the orographic uplift of air charged with moisture from the Tyrrhenian Sea, and to Balkan influence on the latter. The Ionian area is characterized by open environment taxa (e.g. Bos primigenius) while the Tyrrhenian one shows an abundance of forest species (Cervidae).

\subsubsection{Central - southwestern (Tyrrhenian) Italy}

Southwestern Italy (Tyrrhenian Area - Table 1, Fig. 1 - Area 2) is best represented by Grotta di Castelcivita (Salerno). This site is located $94 \mathrm{~m}$ asl and is about $20 \mathrm{~km}$ far from the modern coastline, in a territory encompassing the valley of the Calore river and the Alburni mountains (m 1742). The archaeological sequence is dated to MIS 3 (Gambassini, 1997) and is about $2.5 \mathrm{~m}$ thick. The lowermost portion (layers cgr, gar, lower rsi, spits 32-18lower) contains Late Mousterian deposits and is overlaid by Uluzzian layers (upper rsi, pie, rpi, rsa", spits 18upper-10lower). The sequence is capped by Protoaurignacian layers (rsa'-gic-ars, spits 10upper-top of sequence), which are sealed by the Campanian Ignimbrite (Giaccio et al., 2017). From a zooarchaeological point of view, a unique aspect of this site is the presence of freshwater fish in all chronological phases (Cassoli and Tagliacozzo, 1997).

Grotta della Cala (Marina di Camerota - Salerno) opens close to the present coastline into a steep calcareous cliff which is part of a hilly/ mountain range characterized by plateaus and valleys. The MIS 3 coastline was about $5 \mathrm{~km}$ from the cave entrance. The stratigraphic sequence is about $3 \mathrm{~m}$ thick and starts from the bottom with Middle Palaeolithic layers in a succession of stalagmites and clastic sediments (Martini et al., 2018). At the entrance of the cave, the Middle Palaeolithic is followed by early Upper Palaeolithic deposits, containing Uluzzian (spit 14) and Protoaurignacian (spits 13-10) evidence (Benini et al., 1997; Boscato et al., 1997). These are covered, after a stratigraphic hiatus, by Gravettian, Epigravettian, Mesolithic and Neo-Eneolithic layers (Palma di Cesnola, 1993).

Beyond these well-documented sites, the only other Uluzzian deposit with faunal assemblages in the region is documented at the Tuscan site of Grotta la Fabbrica (Grosseto; Pitti et al., 1976). Here the abundance of equids points to open environments (less evident in the Protoaurignacian layers). As far as the Late Mousterian is concerned, a similar faunal composition is recorded at Buca della Iena (Lucca; Stiner, 1994). Cervidae are, in contrast, the most abundant family in coeval deposits of Grotta dei Santi (Monte Argentario, Grosseto), suggesting a more humid/temperate climate. In Latium a temperate/humid phase connoted by abundant aurochs and deer remains is recorded at Grotta del Fossellone (Alhaique and Tagliacozzo, 2000) and at Grotta di S. Agostino (Stiner, 1994). A similar trend is found at Grotta Breuil (Alhaique and Tagliacozzo, 2000) where Cervidae are the most abundant in layers 6 and 3a, although ibex remains become more frequent in the latter. In Campania, at Riparo del Poggio (Marina di Camerota), located near Grotta della Cala, faunal assemblages are dominated by Cervidae and are typically linked to temperate climates (fallow deer is the most abundant species; Boscato et al., 2009).

\subsubsection{Central - southeastern (Adriatic-Ionian) Italy}

In the Ionian area (Table 1, Fig. 1 - Area 3) Grotta del Cavallo opens into the rocky coast of Uluzzo Tower Bay, at the margin of a vast rolling plain. This cave contains a 7- meter thick stratigraphy which has at its bottom a marine conglomerate attributed to MIS 5e. This is overlaid by a Mousterian sequence dated between MIS 5 and MIS 3 (Table 1) (layers N-FI). These layers are followed by an Uluzzian sequence (EIII - DIb; Moroni et al., 2018) sandwiched between two tephra layers (Fa and CII) attributed to the Y-6 and the Y-5 (Campanian Ignimbrite) events, respectively (Zanchetta et al., 2018).

Riparo l'Oscurusciuto opens inside the ravine of Ginosa (Taranto), to the north of the modern village and about $20 \mathrm{~km}$ from the present coastline (Fig. 1). The zooarchaeological data suggest that Neandertal hunters exploited both the main regional environments, i.e. forest steppe located on flat hills and forested area on the humid bottom of the gorge.

The Middle Palaeolithic stratigraphy is 6-m thick. A tephra (US 14) attributed to the Green Tuff of Monte Epomeo (Ischia) and dated to ca. $55 \mathrm{ky}$ seals the surface of a living floor currently under excavation (US 15) (Boscato et al., 2004, 2011; Boscato and Crezzini, 2006, 2012; Boscato and Ronchitelli, 2008). All the cultural assemblages investigated can be referred to MIS 3 and fall in a chronological interval of ca. 12,000 years. Recurrent Levallois is the most abundant lithic production system (Marciani et al., 2016, 2018; Spagnolo et al., 2016, 2018).

In Molise (Adriatic area) Grotta Reali (Rocchetta a Volturno) yielded Late Mousterian assemblages mostly consisting of Cervidae that can be linked to cold and humid climatic conditions (Sala et al., 2012).

\section{Materials and methods}

Of all the archaeological contexts mentioned in the introduction, the present research only focuses on the 9 ones that present with quantitative evidence on the distribution of faunal assemblages in Middle-toUpper Palaeolithic transition deposits across Italy ( $>$ 50-35 ky, Tables 1 and A.9 and Fig. 1). Sampled archaeological sites were grouped into three geographic areas based on site location and ecological/environmental context: 1) Northeastern Italy (4 sites); 2) Southwestern/Tyrrhenian Italy (2 sites) and 3) Southeastern/Ionian Italy (3 sites; Fig. 1). New zooarchaeological data for Northwestern Italy are now available from Riparo Bombrini (Pothier Bouchard et al., in this issue), while for the southeastern/Ionian area the zooarchaeological analysis from Grotta di Uluzzo C is currently in progress (Fig. 1). Both sites are part of the ERC n. 724046 - SUCCESS project, but they are not included in the present synthesis.

All faunal remains used to compute species abundance based on taxon frequency were uncovered by sieving sediment using $0.5 \mathrm{~mm}$ and $1 \mathrm{~mm}$ meshes in Northeastern, Southwestern, and Southeastern Italy. Based on currently available evidence, specimens were nonetheless grouped into three size classes in Northeastern Italian contexts $(0.1-1 \mathrm{~cm}, 1-3 \mathrm{~cm},>3 \mathrm{~cm}$; Table 2) and into two size classes in Southwestern and Southeastern Italian contexts $(1-3 \mathrm{~cm},>3 \mathrm{~cm}$; Table 5).

Different sources of surface bone alteration (anthropic cut marks vs. animal tooth marks, trampling, postdepositional and modern modifications generated during excavation) were discriminated drawing criteria outlined in a on well-established body of taphonomic literature (Binford, 1981; Potts and Shipman, 1981; Shipman and Rose, 1984; Blumenschine and Selvaggio, 1988; Capaldo and Blumenschine, 1994; Lyman, 1994; Blumenschine, 1995; Fisher, 1995; Fernández-Jalvo and Andrews, 2016; Duches et al., 2016). The degree of combustion was estimated using the method developed by Stiner et al. (1995) and, in Northeastern Italian contexts, burned and calcined bones were separated from unburned materials.

Faunal remains were attributed to species and genus and, when these were not determinable, to families. Unidentified mammal bones were grouped into three classes based on body size: large (red deer, moose, giant deer, bison, aurochs, horse, lion and bear); medium (alpine ibex, chamois, roe deer, fallow deer, wild boar, wolf, lynx, leopard and hyena); and small (hare, marmot, beaver, mustelids, wild cat and 
Table 2

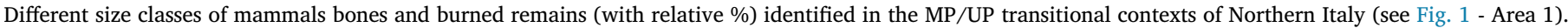
$\mathrm{RF}=$ Grotta di Fumane; $\mathrm{RB}=$ Riparo del Broion; $\mathrm{SB}=$ Grotta di San Bernardino; RS = Grotta del Rio Secco.

\begin{tabular}{|c|c|c|c|c|c|c|c|c|c|c|c|c|c|c|}
\hline US - Levels & Technocomplex & $0.1-1 \mathrm{~cm}$ & $\%$ & $1-3 \mathrm{~cm}$ & $\%$ & $>3 \mathrm{~cm}$ & $\%$ & TOTAL Rem. & Burn. + Calc. & $\%$ & Burned & $\%$ & Calcined & $\%$ \\
\hline RF A2-A2R & PA & 13042 & 65.8 & 6280 & 31.7 & 507 & 2.6 & 19829 & 7861 & 40 & & & & \\
\hline RF A3 & UL & 7831 & 46.1 & 8231 & 48.4 & 927 & 5.5 & 16989 & 4723 & 28 & 2840 & 60.1 & 1883 & 39.9 \\
\hline $\mathrm{RB} 1 \mathrm{e}+1 \mathrm{f}+1 \mathrm{~g}$ & UL & 33199 & 88.8 & 3748 & 10 & 443 & 1.2 & 37390 & 18464 & 49 & 15595 & 84.5 & 2869 & 15.5 \\
\hline RF A4 & LM & 9770 & 49 & 9287 & 46.5 & 898 & 4.5 & 19955 & 7321 & 37 & 5187 & 70.9 & 2134 & 29.1 \\
\hline SB II + III & LM & 2744 & 29.8 & 5337 & 57.9 & 1136 & 12.3 & 9217 & 5431 & 59 & 4747 & 87.4 & 684 & 12.6 \\
\hline RS 5top +7 & LM & 43 & 8.2 & 47 & 9 & 434 & 82.8 & 524 & 693 & 8 & 42 & 97.7 & 1 & 2.3 \\
\hline $\mathrm{RF}$ A5/A5+A6 & LM & 35342 & 52.7 & 29767 & 44.4 & 1974 & 2.9 & 67083 & 38255 & 57 & 30442 & 79.6 & 7813 & 20.4 \\
\hline RF A6 & LM & 62692 & 56.5 & 43944 & 39.6 & 4408 & 4 & 111044 & 53413 & 48 & 46854 & 87.7 & 6559 & 12.3 \\
\hline RF A9 & LM & 78119 & 69.8 & 30763 & 27.5 & 2959 & 2.6 & 111841 & 54411 & 49 & 50398 & 92.6 & 4013 & 7.4 \\
\hline RS $5+8$ & LM & 2307 & 53.6 & 1538 & 35.8 & 456 & 10.6 & 4301 & 43 & 16 & 671 & 96.8 & 22 & 3.2 \\
\hline
\end{tabular}

fox). In addition, unidentified specimens from the southern sites were grouped according to anatomical categories such as "skull", "jaw", "teeth", "vertebrae", "ribs" etc. or more general categories such as "epiphysis" "diaphysis" and "spongy bones".

As far as northern Italy is concerned, taxonomic and skeletal identification were based on the reference collections stored at the Bioarchaeology Section of the National Prehistoric Ethnographic Museum "Luigi Pigorini" (Lazio Museum Pole, Rome), at the Prehistoric and Anthropological Sciences Section in the Department of Humanities, University of Ferrara and at the Laboratory of Osteoarchaeology and Palaeoanthropology at the Department of Cultural Heritage, University of Bologna (Ravenna). Bone assemblages recovered from the southern Italian sites were compared with the reference collection stored at the Research Unit of Anthropology and Prehistory of the University of Siena. Differences between the Uluzzian layer of Grotta del Cavallo and the Late Mousterian layers at Grotta del Cavallo and Riparo l'Oscurusciuto (i.e. the only layers which displayed no sign of carnivore activity on ungulate bones) were formally assessed for percentages of carpal and tarsal bones, and of phalanges and sesamoides (relative frequencies were based on both total ungulate counts and on the remains of Bos Primigenius). In addition, the presence of significant differences was tested for remnant diaphysis, epiphysis, and spongy bones between the same layers. To do so we measured effect size as Cohen's $h$ using the function ES.h in the package pwr in R (Champely, 2018), we then measured statistical power using the dedicated pwr.2p2n.test function in the same package, and performed a two-tailed test for equality in proportions between the chosen layers (with continuity correction for cases in which the number of successes or failures was lower or equal to 5; Tab. A.13 - A.17). We also tested the hypothesis of differences in the degree of fragmentation across sites of Northern Italy by running arcsine transformation of proportions fragment-size classes at all sites (1-3 cm, > $3 \mathrm{~cm}$; following Morin et al., 2019) and then comparing the distribution of transformed values between Uluzzian and Late Mousterian layers via a two-tailed Mann-Whitney test for independent sample design. As for southern Italy, we once again only focused on Riparo l'Oscurusciuto and Grotta del Cavallo in Southeastern Italy. We tested for significant differences in proportions and also calculated effect size and statistical power to support the obtained results.

Species abundance was quantified using the Number of Identified Specimens (NISP; Grayson, 1984). Notwithstanding its limitations (e.g. inflation of the count of rare parts, lower predictive power when limited to long bones), this particular species estimate offers accuracy and reproducibility at the ratio scale (Morin et al., 2017). The ubiquitous recording of NISP in all the examined contexts made it the best available method to directly compare different sites across the study region. Once NISP of each mammal group or species was obtained for all layers of the 9 sampled archaeological sites across Italy, we grouped layers belonging to the same region (i.e. Northeastern, Southwestern, and Southeastern Italy) and within each region we ordered them into a single diachronic sequence, based on absolute dates (Tables 1 and A.9) and associated material cultural evidence. Avifaunal remains were comapred only for Fumane and Castelcivita caves for abundance reasons and lack of comparable sequences in any other sites. Relative taxon abundance was calculated in each layer and variability in relative frequency over time was inspected through bar charts, in order to highlight any differences between trends emerging in different regions.

Comparing NISP proportions across different archaeological layers (in the same context or between different contexts), however, presents a number of potential issues. In addition to post-depositional processes, substantial differences in the time of accumulation of different layers may have deleterious effects on the accurate representation of faunal spectra. This process, known as time-averaging, is extremely frequent in geologic and anthropic contexts (Binford, 1981; Kowalewski, 1996; Premo, 2014; Madsen, 2018), and has a direct impact on the reliability of the quantification of abundance, richness, evenness, and diversity in time-averaged samples (Leonard and Jones, 1989). Specifically, the longer the duration of layer formation, the more inflated richness and diversity will be. This makes tracking change over time more problematic and increases the risk of misidentifying inflated counts for actual human choices (i.e. Type I error when testing hypotheses; Premo, 2014; Madsen, 2018). The presence of differential accumulation rates, palimpsests, and taphonomic processes therefore complicates any attempt at quantifying the effective temporal scale of individual layers solely based on stratigraphy.

In addition, inference made by comparing NISP proportions is hampered by the limitations of closed datasets (Lyman, 2008; Orton et al., 2017). Species relative frequencies are by definition computed over the total number of collected remains and their sum is bound to be equal to 1 . No relative frequency is free to vary over time without affecting or being affected by change in the frequency of another class, i.e. the relative abundance of one particular taxon will always be negatively correlated to the relative abundance of another taxon. Interpreting such increases and decreases as the effect of some independent mechanism (e.g. environmental change, cultural selection) is therefore not always straightforward.

In order to overcome the limitations mentioned above while providing support for the trends that might emerge from relative taxonomic abundance analysis across the time-ordered layers of different sites, we also built long-term time-series of zooarchaeological data 
documented at Grotta di Fumane (Northeastern Italy) that can directly be compared against independent sources of information (e.g. palaeoclimatic models, palinological and palaeoenvironmental data), and across mismatched and differentially overlapping contexts. Grotta di Fumane was chosen as a case study because it offers the longest and best-dated sequence among all the available sites.

More specifically, we computed aoristic sums (i.e. the sum of the probability of existence of all events for a given temporal interval) of taxon abundance to obtain estimates of taxon frequency based on absolute radiocarbon dates. Aoristic analysis has been already employed in a few archaeological and zooarchaeological studies (Ratcliffe, 2000; Johnson, 2004; Crema, 2012; Bevan et al., 2013; Orton et al., 2017), although the method is still generally rarely used and, to the best of our knowledge, it has never been applied to palaeolithic contexts. This approach consists of: a) assigning a start and end date to each archaeological layer from which fossil fragments had been retrieved; b) dividing the entire time span of the study period into temporal bins of fixed width; c) based on the start and end dates of the relevant layer, and drawing on Laplace's principle of insufficient reason (see Crema, 2012; Orton et al., 2017 for a detailed discussion), dividing the total probability mass of each deposition event/fragment (equal to 1 ) across the $t$ temporal bins comprised in the date interval of the layer. Each deposition event therefore exhibits a uniform probability of existence at each bin calculated as $1 / t$; d) summing all the probabilities falling in the same bin, and repeating the same operation for the entire study period.

The result is an estimate of species frequency distribution which incorporates all the temporal uncertainty embedded in the data. Better dating leads to shorter temporal intervals for each deposition event, that in turn allows researchers to assign a higher probability of existence at each temporal bin. As a consequence, worse dating leads to higher dispersion in the probability of existence, i.e. to stable time series which do not show clear evidence of increase or decrease as an artefact due to lack of resolution. In the present work, we first set the temporal limits for each layer at Grotta di Fumane. When start and end dates were already available from the literature (as in the case of layers A9) these intervals were directly taken (Table A.9). As far as all the remaining layers are concerned (A6, A5/A5 + A6, A4, A3, and A2), start and end dates were calculated in OxCal 4.3 (Bronk Ramsey, 2009) as the median of the $68.2 \%$ interval taken from the posterior probability distribution of already published layer boundaries (Higham et al., 2014). This particular model was chosen to fully exploit the potential of aoristic analysis and considering that at this site Uluzzian and final Mousterian are reported as temporally indistinguishable (Douka et al., 2014).

Raw NISP counts were then used to compute aoristic sums of each taxon across 50-year bins through the function aorist in the package archSeries in $\mathrm{R}$ version 3.4.4 (Orton, 2017, $\mathrm{R}$ Core Team 2018). To avoid generating artifacts due to empty bins at the interval $41600-41100 \mathrm{cal}$ $\mathrm{BP}, 10$ years were added to the median date for the end boundary of level A3. Taxon-specific aoristic values were then summed and used to calculate estimates of taxon relative frequencies. To further ascertain the presence of absolute shifts in estimated frequency, we also plotted the aoristic sum of ungulates. In this case, absolute frequency estimates were compared against 95\% confidence envelopes generated through Monte-Carlo simulation ( $\mathrm{n}$. iterations $=5000$ ) as well as against a dummy model generated assuming a uniform frequency distribution following Crema (2012) and Orton (Orton et al., 2017). Both the empirical and dummy simulations were computed using the function $d a$ te.simulate in the package archSeries. Using the same function, rates of change were also computed for ungulate families. The aim was to assess whether there were temporal bins exhibiting shifts in the abundance of families compared to other bins. Following Crema (2012) and Orton (2017), rates of change were examined by observing (in this case through boxplots) the distribution of simulated standardised differences between each chronological bin and the preceding one. Temporal intervals with median and interquartile range falling above the zero line (suggesting stability or absence of change) were interpreted as a sign of increase, while boxes falling under the zero line were interpreted as instances of decrease. Such distributions were compared against the $95 \%$ confidence envelopes of the null model based on the aoristic sum of carnivores, which provides a null expectation independent from palaeoenvironmental change.

\section{Results}

Northeastern Italian contexts yielded a total of 323,964 remains (NISP $=9044$ ) while for Southern Italy as a whole 33,340 remains were documented (NISP $=2351$ ). From a zooarchaeological point of view, Late Mousterian layers have been investigated more intensively than later ones in both regions. Despite the difference in absolute counts, the proportion of mammal orders and classes is roughly the same across all contexts (Fig. 2), with ungulates being the most abundant category followed by carnivores, birds (at Grotta di Fumane and Castelcivita), and rodents, in decreasing order of importance.

Uluzzian layers exhibit an increase in the relative abundance of carnivore and bird remains, matched by a considerably lower number of remains attributed to large rodents (e.g. marmot and beaver) and lagomorphs (Fig. 2). Protoaurignacian phases invert this trend, with an appreciable decrease in the number of carnivore and bird remains.

\subsection{Mammals}

Despite the specificities that may bias the abundance of faunal remains in each of the examined contexts (e.g., Grotta del Rio Secco being consistently used by bears which, in turn, were routinely exploited by Neandertals; Romandini et al., 2018b), most of Late Mousterian levels and layers in Northeastern Italy show an increase in the prevalence of cervidae, followed by a decrease of Cervus elaphus and Capreolus capreolus matched by a gradual increase, in the Uluzzian and Protoaurignacian, in caprinae, especially Capra ibex and Rupicapra rupicapra (Fig. 3 and Table A.1). This change over time in the relative abundance between cervidae and caprinae may hint at a shift from a temperate climate characterised by forests and meadows to an alpine setting with open environments.

The archaeological sites are located in a region that included habitats suitable for bovinae, ranging from dense forests with wetlands and small streams more attractive to Bos primigenius, to hilly grasslands and plains, populated by bison. However, bovids are generally less abundant than the previous families, and their presence remains roughly constant across the entire study sequence.

Moose (Alces alces) and giant deer (Megaloceros giganteus) are less frequent and well attested in Mousterian and Uluzzian layers. Their presence suggests - during this period - the existence of humid woodlands near the sampled archaeological sites. Wild boar is rarer yet, being present anecdotally in the Late Mousterian at Grotta di Fumane, while it is more abundant at lower elevations (Grotta di San Bernardino, Mousterian Units II + III; Riparo del Broion, Uluzzian layers $1 \mathrm{e}+1 \mathrm{f}+1 \mathrm{~g}$ ). The presence of woolly rhinoceros (Coelodonta antiquitatis) in the Uluzzian layer A3 at Grotta di Fumane and of Stephanorhinus sp. at Grotta di San Bernardino indicates decreasing temperatures and presence of cold arid conditions.

In this region, carnivores are quantitatively more represented in Late Mousterian and Uluzzian assemblages, while their frequency steadily decreases in Protoaurignacian layers (Fig. 2). Nevertheless, variety of carnivores taxa increase beginning with the Uluzzian (Romandini et al., 2018a), and the presence of wolverine (Gulo gulo), ermine (Mustela erminea), and arctic fox (Alopex cfr. lagopus) further supports the onset of colder and arid climate conditions during the MPUP transition (Fig. 4 and Table A.2). Rodents and lagomorphs (Table A.3) are represented by beaver and marmot, already present in Late Mousterian assemblages, and by lagomorphs in the Uluzzian and Protoaurignacian (Romandini et al., 2018a). Upper Palaeolithic contexts 
also yielded remains of Lepus cfr. timidus, further supporting the diffusion of increasingly colder environments in the latest phase of the studied sequences.

In Southwestern/Tyrrhenian Italy, the Late Mousterian sequence at Grotta di Castelcivita (spits 32-24) yielded a conspicuous amount of cervidae fragments (Cervus elaphus, Dama Dama, Capreolus capreolus); fallow deer in particular is the most abundant species (Fig. 5 and Table A.4). Later on, in spits 23-18 lower, there is an increase in the abundance of red and roe deer and of chamois (Rupicapra sp.), correlated to an increase in humidity (Masini and Abbazzi, 1997). The beginning of the Uluzzian sequence (spits 18 upper-15) is characterised by higher frequencies of horse (Equus ferus) and large bovids (Bison priscus and Bos/Bison) suggesting the occurrence of colder climates and sparse woodland. In the following Uluzzian layers (spits 14-10 lower), an additional increase in the occurrence of equids and a decrease in the frequency of fallow deer suggest more open environments. The Early Protoaurignacian (spits 10 upper -8 lower) shows comparable environmental conditions, while spits 8upper-7 can be linked to an increase in woodland cover as suggested by the higher presence of deer and the decrease in the frequency of horse (Fig. 5 and Table A.4), followed by cold-temperate phases (spit 6) (Masini and Abbazzi, 1997). The anthracological evidence supports the climatic and ecological trend inferred from zooarchaeological remains (Castelletti and Maspero, 1997).

At Grotta della Cala (Marina di Camerota, Salerno), faunal remains from the Uluzzian (spit 14) are characterised by a conspicuous presence of cervidae (representing on the whole $74 \%$ of ungulates) and in particular of fallow deer, typical of temperate climates and Mediterranean evergreen forest. In the Protoaurignacian layers (spits 13-10), lower frequencies of fallow deer and higher frequencies of red deer indicate the onset of colder conditions (Fig. 5 and Table A.4). Low frequencies of ungulates linked to open environments/wooded steppe (such as horse, alpine ibex and aurochs) are also recorded (Benini et al., 1997). Cervids account for over $70 \%$ of the ungulates recovered in these layers (Boscato et al., 1997).

In the same region, carnivores occur in all phases. Whilst in the Middle Palaeolithic, most of the remains are referable to the spotted hyaena and the leopard, species richness increase in the Uluzzian and in the Protoaurignacian (Table A.5). Rodents and lagomorphs are very rare.

The record of Southeastern/Ionian Italy, on the other hand, is based on the sequences uncovered at Riparo l'Oscurusciuto (Ginosa - Taranto) and Grotta del Cavallo (Nardò - Lecce) (Figs. 1 and 6 and Tables A.6). At Riparo l'Oscurusciuto, layers 13:4 are characterised by the substantial presence of Bos primigenius, counterbalanced by low frequencies of horse, rhinoceros and caprinae, and by anecdotal frequencies of cervidae (especially fallow deer), all of which hints at an environment characterised by wooded meadows and open spaces (Fig. 6). Aurochs is less frequent in SU 3, while in the same unit, deer is more abundant, the presence of rhinoceros can be inferred by tooth fragments, and horse

\section{NORTHERN ITALY}

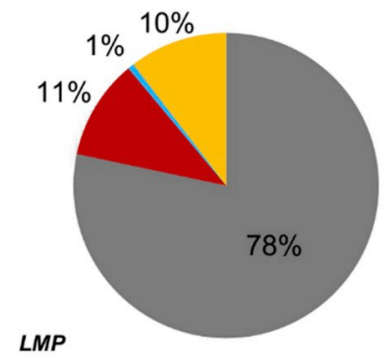

UNGULATA CARNIVORA RODENTIA - LAGOMORPHA AVES

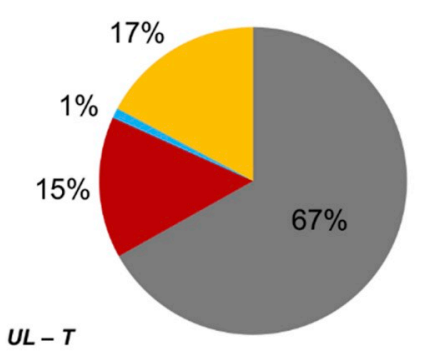

$U L-T$

UNGULATA

CARNIVORA

RODENTIA - LAGOMORPHA AVES

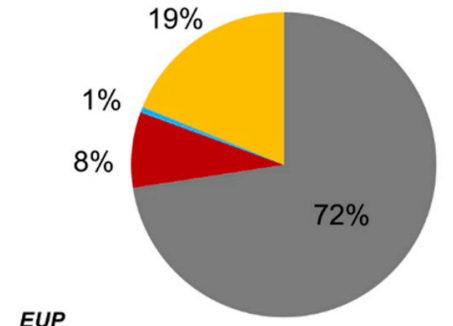

EUP

$\begin{array}{lr}\text { UNGULATA } & 950 \\ \text { CARNIVORA } & 105 \\ \text { RODENTIA - LAGOMORPHA } & 8 \\ \text { AVES } & 246\end{array}$
SOUTHERN ITALY

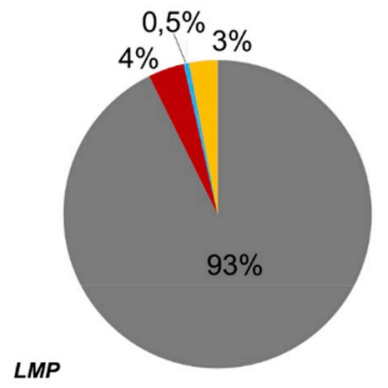

UNGULATA

CARNIVORA

RODENTIA - LAGOMORPHA

AVES

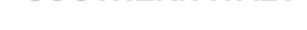

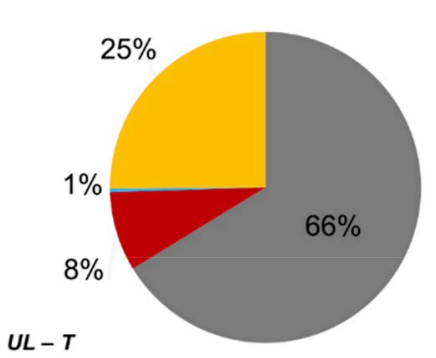

UNGULATA

CARNIVORA

RODENTIA - LAGOMORPHA

AVES
961

941

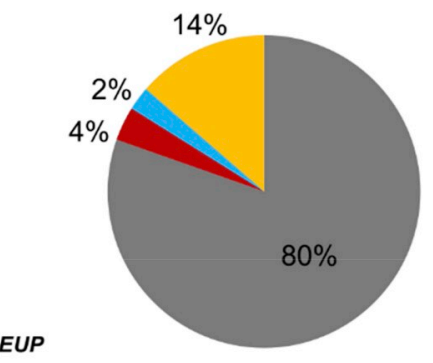

UNGULATA

CARNIVORA

RODENTIA - LAGOMORPHA

AVES
979
43
30

30
166

$\because$ UNGULATA $\because$ CARNIVORA

$\square$ RODENTIA - LAGOMORPHA $\square$ AVES

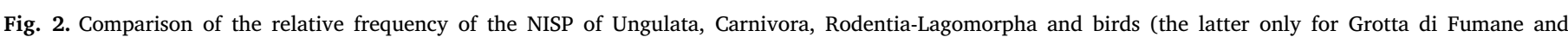

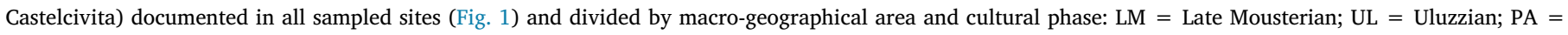
Protoaurignacian. 
becomes the most represented ungulate (Table A.6). At the end of the sequence (SU2-1), aurochs is once again the most abundant ungulate, while the increase in abundance of fallow deer suggests the onset of a temperate phase (Boscato and Crezzini, 2012).

The Late Mousterian sequence at Grotta del Cavallo (layers FIII-FI) also yields evidence of the climatic fluctuations known for MIS 3 (Table A.6), which agrees with the sequence described for Riparo l'Oscurusciuto. An initial phase characterised by open/steppic forests indicated by the dominance of aurochs remains (layer FIIIE) is followed, in layers FIIID-FIIIB, by a more temperate phase (as suggested by higher frequency of fallow deer) and by a third, more arid stage in layers FIII-FI associated with the presence of aurochs and horse (Sarti et al., 2000, 2002) (Table A.6). The lowermost Uluzzian level EIII5 suggests, in contrast, a shift to an increasingly colder climate with more dispersed woodlands, indicated by the absence of fallow deer and by the increased presence of horses (Table A.6; Boscato and Crezzini, 2012).

With the only exception of red fox which has been found in the Late Mousterian of Grotta del Cavallo, carnivores, rodents and lagomorphs are almost absent in the assemblages of Ionic area (Table A.7).

\subsection{Avifaunal remains}

Substantial evidence on the exploitation of avifauna was documented for Grotta di Fumane and Grotta di Castelcivita (Cassoli and Tagliacozzo, 1994a, 1997; Masini and Abbazzi, 1997; Gala and Tagliacozzo, 2005; Peresani et al., 2011a; Romandini, 2012;

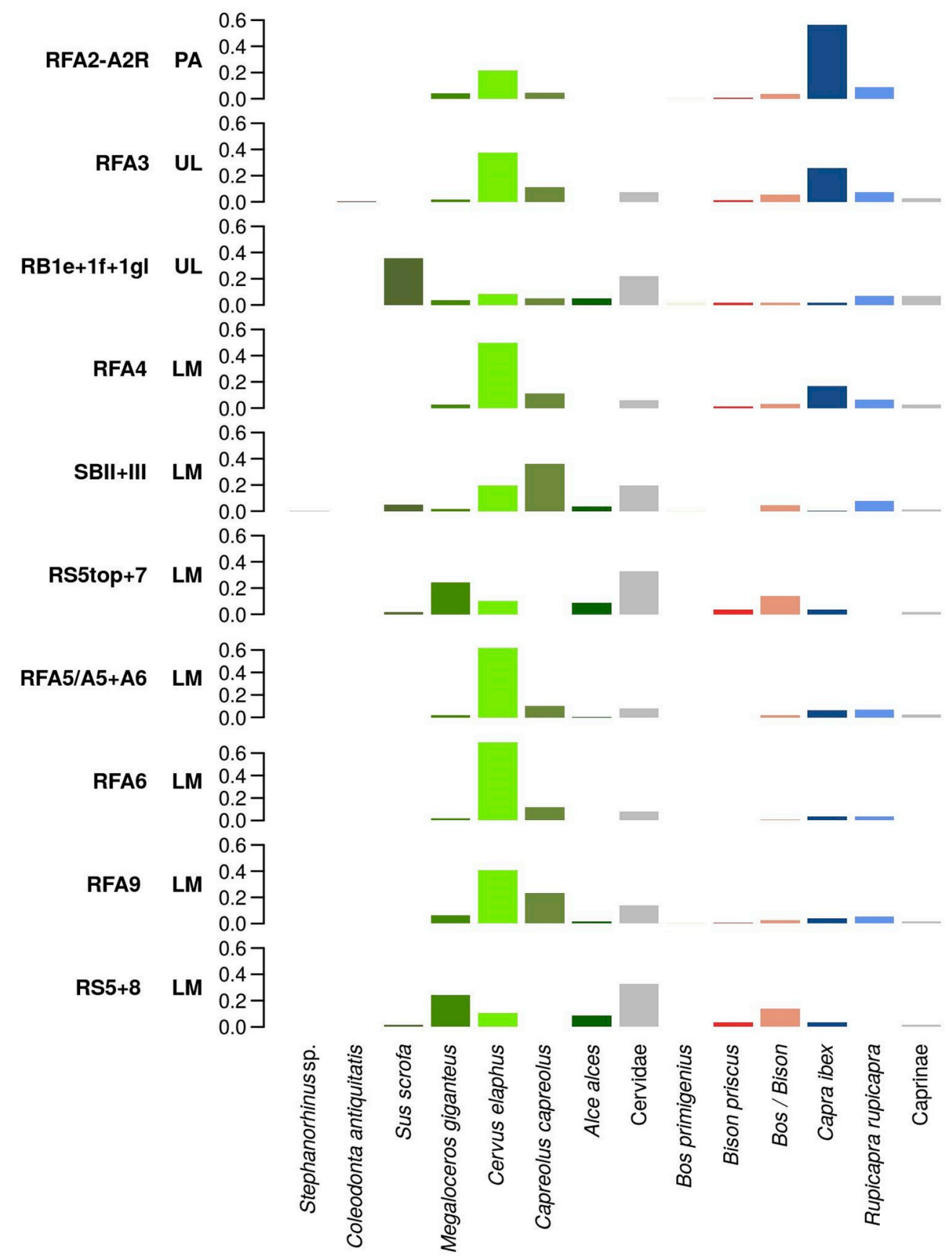

Fig. 3. Bar charts showing the relative contribution of each ungulate taxon to the total NISP recorded in the different levels and layers sampled in Northern Italy. The contexts are in chronological-cultural order (from bottom to top) based on the archaeological sequence of each site. RF $=$ Grotta di Fumane; RB = Riparo del Broion; $\mathrm{SB}=$ Grotta di San Bernardino; RS = Grotta del Rio Secco. LM = Late Mousterian; UL = Uluzzian; PA = Protoaurignacian. 
Tagliacozzo et al., 2013; Romandini et al., 2016a, b; Gala et al., 2018; Fiore et al., 2004, 2016, 2019).

The bird species identified at Grotta di Fumane belong to the extant Italian avifauna (Table A.8) with the exception of the willow grouse ( $L$. cf. lagopus), a boreal species which has historically never been observed in Italy. The parrot crossbill (L. pytyopsittacus) is another boreal species currently found in Northern Europe and considered a vagrant species in Italy (Brichetti and Fracasso, 2015). Taxa linked to open and rocky environments are the most abundant (Fig. 7). More specifically, bearded vulture ( $G$. barbatus), golden eagle (A. chrysaetos), red-billed and yellow-billed chough (P. pyrrhocorax and P. graculus), common raven (C. corax), Eurasian crag martin ( $P$. rupestris) and white-winged snowfinch (M. nivalis) indicate the presence of rocky cliffs, while treeless terrain with rocky outcrops is indicated by rock partridge ( $A$. graeca) and rock ptarmigan (L. muta). On the other hand, the presence of wooded areas in the surroundings of the cave is indicated by the black grouse (L. tetrix), stock dove (C. oenas), common woodpigeon ( $C$. palumbus), Boreal owl (A. funereus), tawny owl (S. aluco), white-backed woodpecker (D. leucotos, currently reduced to small populations in the Central Apennines but once more widespread; Pavia, 1999; Brichetti

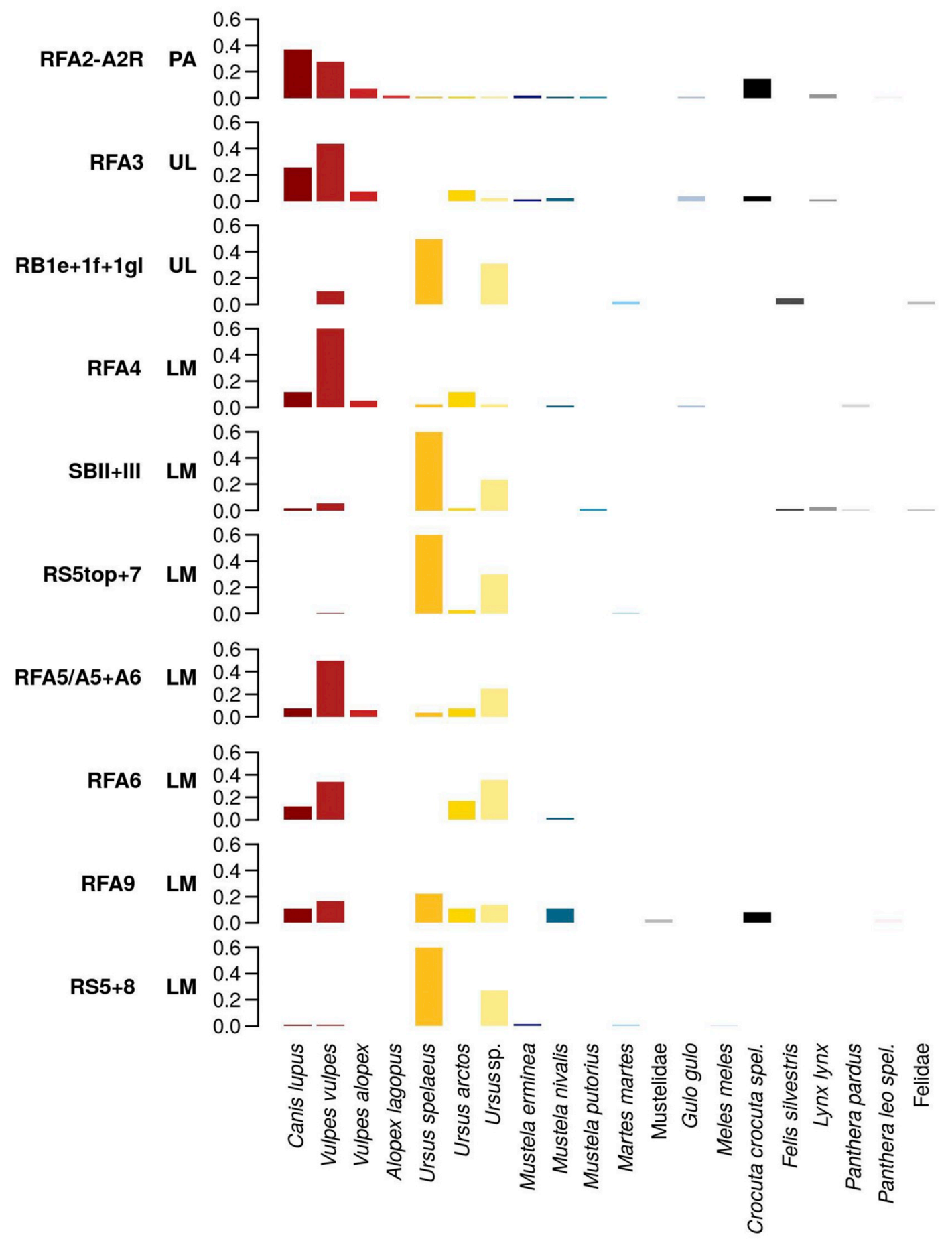

Fig. 4. Bar charts showing the relative contribution of each carnivore taxon to the total NISP recorded in the different levels and layers sampled in Northern Italy. The contexts are in chronological-cultural order (from bottom to top) based on the archaeological sequence of each site. RF $=$ Grotta di Fumane; RB = Riparo del Broion; $\mathrm{SB}=$ Grotta di San Bernardino; RS = Grotta del Rio Secco. LM = Late Mousterian; UL = Uluzzian; PA = Protoaurignacian. 
and Fracasso, 2007) Eurasian jay (G. glandarius), and Eurasian bullfinch (P. pyrrhula). The presence of the northern nutcracker ( $N$. caryocatactes) and parrot crossbill (L. pytyopsittacus) also point to the presence of coniferous forests. Additionally, open grasslands and wet meadows are indicated by common quail (C. coturnix), grey partridge ( $P$. perdix), corncrake (C. crex, which was breeding in the surroundings of the cave, as attested by the finding of juvenile bones) and northern lapwing ( $V$. vanellus), while willow grouse ( $L$. cf. lagopus) is an indicative of tundralike open areas such as moors and peatlands. Finally, the presence of two duck species (A. platyrhynchos and A. cf. crecca) and two Rallidae (R. aquaticus and cf. G. chloropus) suggests nearby wetlands or slowflowing water courses (Cramp, 1998).

As a whole the bird assemblage at Fumane points to an Alpine ecological setting with forests and open areas. Several identified bird species (rock ptarmigan, black grouse, Boreal owl, bearded vulture, white-backed woodpecker, red and yellow-billed chough, Northern nutcracker and white-winged snowfinch) currently live in Italy at considerably higher altitudes than Fumane. The presence of their fossil remains at $350 \mathrm{~m}$ asl suggests the downward shifting of the vegetational zones during MIS 3 due to a decrease in climate value parameters. The presence of remains probably belonging to willow grouse in layer A6 and to parrot crossbill in layers A1 + A2 during two of the harsher climatic phases (Heinrich Event 5 and Heinrich Event 4 respectively) (López-García et al., 2015) might be an example of two boreal species seeking a refugium in Mediterranean Europe (Tyrberg, 1991; Carrera et al., 2018a,b).

The relative frequency of species related to forest, open, rocky and water environments calculated for each layer (Fig. 7) suggests the presence of temperate conditions for layer A9, followed by a colder climate in A6. The species linked to open environments decline sharply in layer A4 (attributed to the GI12 interstadial) before increasing anew in layer A3, marking the beginning of Heinrich Event 4 that lasts until

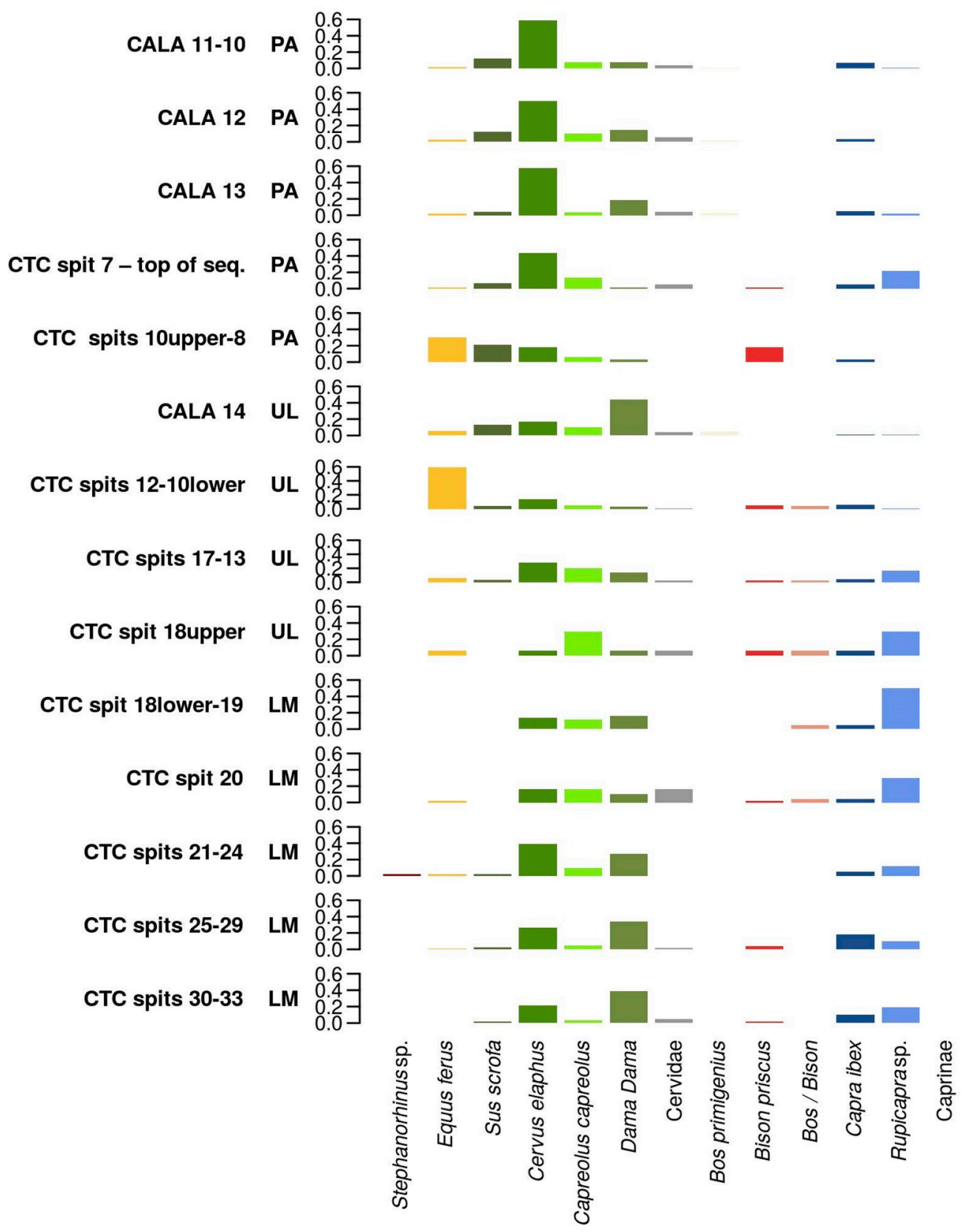

Fig. 5. Bar charts showing the relative contribution of each ungulate taxon to the total NISP recorded in the different levels and layers sampled in Southwestern Italy (Tyrrhenian Area). Contexts are presented in chronological-cultural order (from bottom to top) based on the archaeological sequence of each site. CTC $=$ Grotta di Castelcivita; CALA = Grotta della Cala. $\mathrm{LM}=$ Late Mousterian; UL = Uluzzian; PA = Protoaurignacian. 
the end of the sequence (López-García et al., 2015). Heinrich Event 4, however, did not prevent the persistence of open forests, as attested by the bird taxa found in A1 + A2 and A3. The apparent increase of forest bird taxa in the layers corresponding to Heinrich Event 4 could be explained by a switch from anthropic to natural accumulation in the Protoaurignacian, as suggested by the lack of anthropic marks on bird bones.

Turning to Grotta di Castelcivita, while the bird remains have been the object of a recent taphonomic revision (Fiore et al., 2019), the data discussed here are drawn from Cassoli and Tagliacozzo (1997). All identified taxa belong to the extant Italian avifauna (Table A.8 and Fig. 8, Cassoli and Tagliacozzo, 1997; Brichetti and Fracasso, 2015; Gala et al., 2018). In terms of NISP, the vast majority are from open and rocky environments. The presence of extensive wetlands and marshes near the cave is suggested by several duck, wader and gull species ( $A$. nyroca, S. querquedula, M. strepera, M. penelope, A. platyrhynchos, A. crecca, P. squatarola, N. phaeopus, L. limosa, A. interpres, C. pugnax, G. media, L. ridibundus). The red-billed chough ( $P$. pyrrhocorax), yellowbilled chough ( $P$. graculus) and Eurasian crag martin ( $P$. rupestris) are indicative of rocky cliffs, while rock partridge (A. graeca) attests to the presence of treeless rocky terrain. Open areas such as grasslands, steppe and shrublands were also present, as indicated by the common quail ( $C$. coturnix), grey partridge ( $P$. perdix), Eurasian thick-knee (B. oedicnemus), and little owl (A. noctua). The stock dove (C. oenas) and tawny owl (S. aluco) are associated with wooded areas, while the presence of the northern nutcracker ( $N$. caryocatactes) suggests the presence of coniferous forests (Cramp, 1998) and confirms that, in the past, this species was distributed across a much broader area than today (Gala and Tagliacozzo, 2010; Brichetti and Fracasso, 2011). The surroundings of Castelcivita were therefore characterised during MIS 3 by extensive wetlands in plain areas, and by drier environments (such as grasslands, bare terrains and cliffs) intermingled with conifer or mixed forests at higher elevations. The presence at about $100 \mathrm{~m}$ asl of species that currently live at higher altitudes (rock partridge, choughs and northern nutcracker), suggests colder and harsher conditions during the whole sequence. In the Uluzzian layer rpi, the number of bird taxa related to open environments increases and point to an expansion of grasslands linked to colder and more arid conditions possibly corresponding to the beginning of Heinrich Event 4 (or a preceding stadial), based on currently available dates (Fig. 8). In the Protoaurignacian layers, the riparian taxa slightly increase, as do those of forest environments in layer ars, probably indicating a climatic amelioration at the top of the sequence (Cassoli and Tagliacozzo, 1997; Gala et al., 2018). All phases provided evidence of human exploitation of at least some bird species (Fiore et al., 2019).

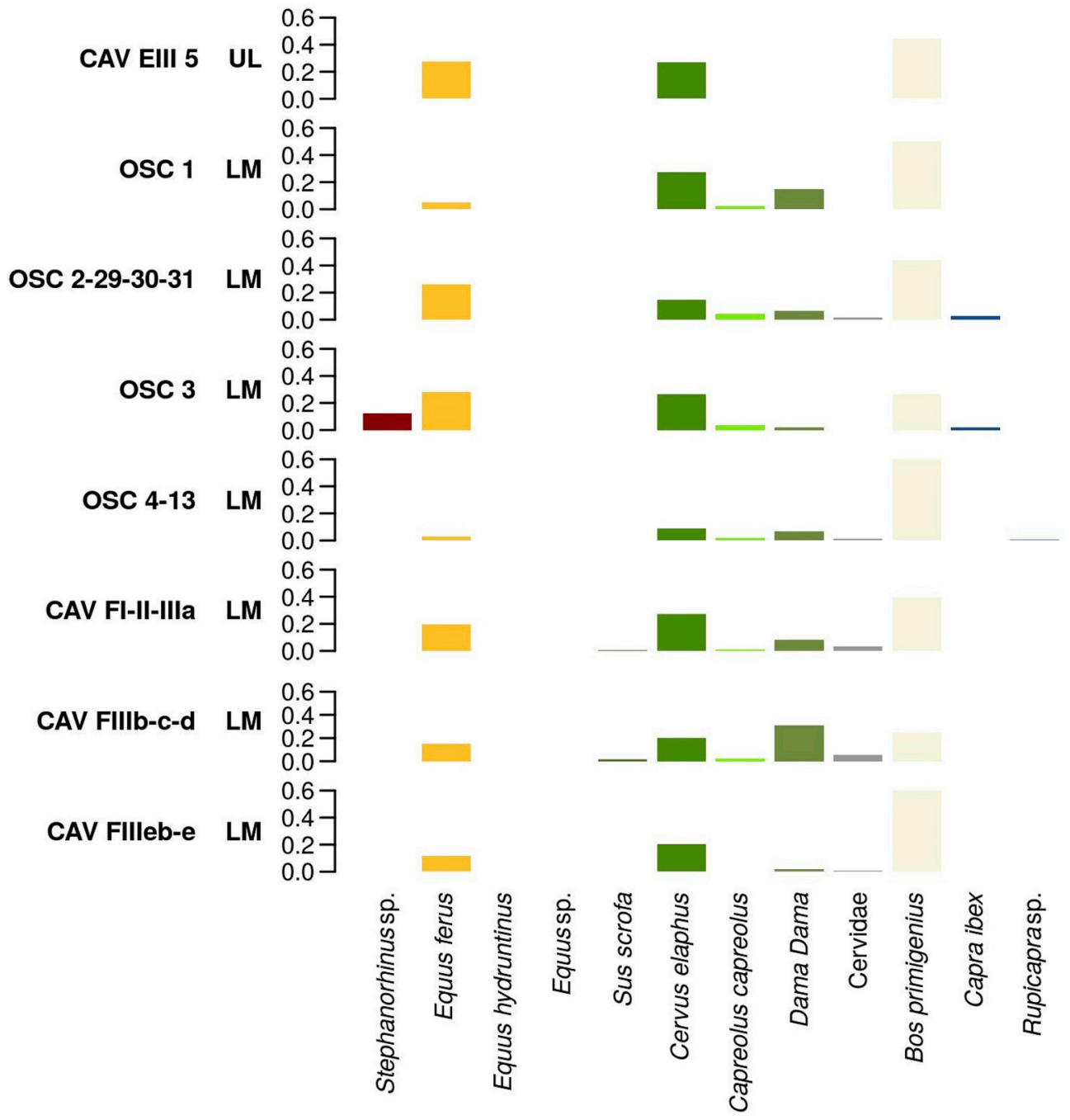

Fig. 6. Bar charts showing the relative contribution of each ungulate taxon to the total NISP recorded in the different levels and layers sampled in Southeastern Italy (Ionian-Adriatic area). Contexts are presented in chronological-cultural order (from bottom to top) based on the archaeological sequence of each site. CAV $=$ Grotta del Cavallo; OSC = Riparo l'Oscurusciuto. LM = Late Mousterian; UL = Uluzzian; PA = Protoaurignacian. 


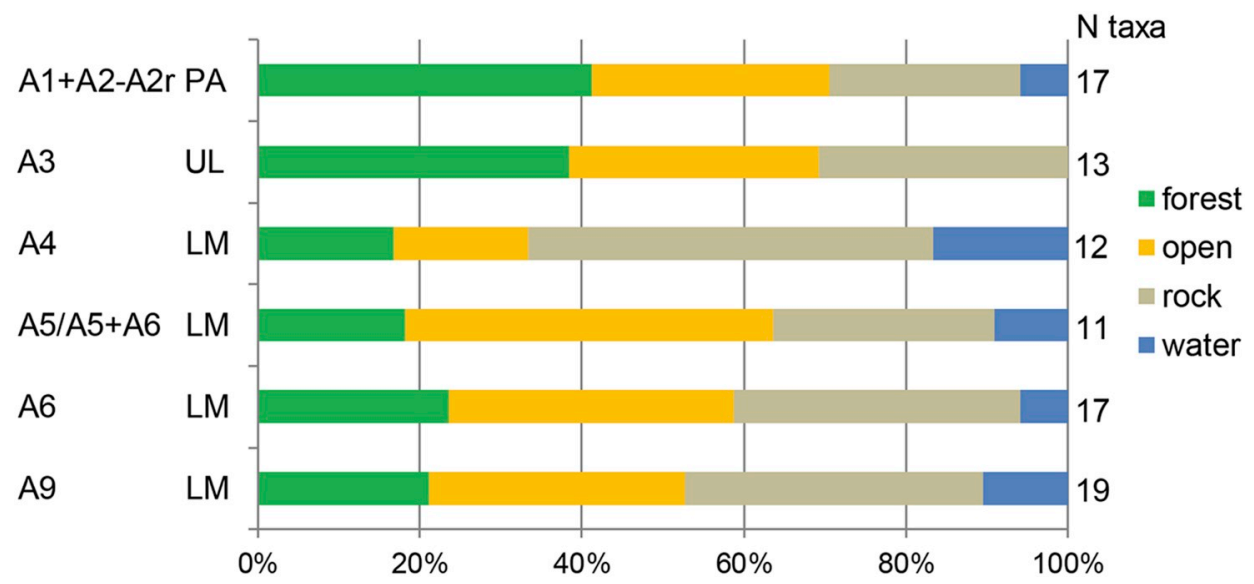

Fig. 7. \% share of the bird taxa of different environments (see legend) in the various layers of Grotta di Fumane, calculated on the total number of bird taxa of each layer. $\mathrm{LM}=$ Late Mousterian; UL $=$ Uluzzian; PA = Protoaurignacian.

\subsection{Aoristic analysis}

As far as estimated relative frequencies of ungulates are concerned, the resulting graph exhibits trends of change over time (Fig. 9 B). The estimates of Capreolus relative abundance are high at $47.6 \mathrm{ky}$ while they decrease after $\sim 45 \mathrm{ky}$. After $\sim 45 \mathrm{ky}$ the estimated remains of Cervus elaphus start becoming more abundant than in previous bins and reach their maximum between $45-44 \mathrm{ky}$. In bins following that date the presence of red deer starts declining in favour of Capra ibex and Rupicapra rupicapra. In the same time interval Bos and Bison show a quick unimodal trend. The apparent stability that emerges after $\sim 40.5 \mathrm{ky}$ it is due to the assumed uniform probability distribution in the absence of additional information on layer chronology. This trend, which can be noticed for all taxa, could be an artefact of uneven chronological sampling, and underscores the great temporal uncertainty associated with Protoaurignacian assemblages.

The distribution of relative carnivore estimates (Fig. 10) shows a marked increase in the presence of Ursidae between $\sim 45$ and $\sim 43.6 \mathrm{ky}$. The relative frequency of wolves becomes higher than that of Ursidae between $\sim 44.1$ and $43.6 \mathrm{ky}$ and becomes the highest value from 41.1 ky onwards. Foxes become most frequent when Ursidae increase and exhibit a inverse trend to that of wolf. From both an environmental and taphonomic point of view, it is interesting to note that after $41.6 \mathrm{ky}$ the estimated relative frequency of Crocuta crocuta spelaea is considerably higher than in previous bins, including bins that record its presence between 47.6-46.6 ka.

Birds adapted to rocky environments are the most frequent at Fumane for the entire study sequence (Fig. 11). At 44.1 ky they exhibit a much lower estimate, while the percentage of avifaunal specimens linked to wooded/forested environments is higher than it was at the beginning of the sequence.

Rates of change based on simulated dates for ungulates (Figs. 9 and 11 ) add interesting elements and support this emerging scenario. When trends for ungulates are plotted against dummy sets based on estimated absolute frequencies of carnivores (i.e. of species with no particular links to change in environmental conditions), simulated $95 \%$ confidence envelopes exceed the expectations of the dummy model confidence area between 47.6-45.6 ky for bovids (Fig. $12 \mathrm{C}$ ). Although median values of simulated rates of change for all three ungulate families never emerge from the $95 \%$ dummy confidence envelope suggesting the lack of significant deviations from a null model of deposition which is supposedly not based on environmental change - boxplots consistently point to the same chronological bins as the interesting ones. More specifically, there is evidence of a possible absolute increase in the frequency of all ungulate families between $\sim 45.6-45.1 \mathrm{ky}$, while a decrease could be hypothesis between 45.1-44.1 ky (see Fig. 12).

Aoristic sum and simulated frequency estimates of all ungulates as a whole (Fig. 9 A) further support the hypothesis that at Grotta di Fumane there was an intensification of the deposition of ungulates between ca 45 and $44 \mathrm{ky}$, coinciding with higher percentages of red deer

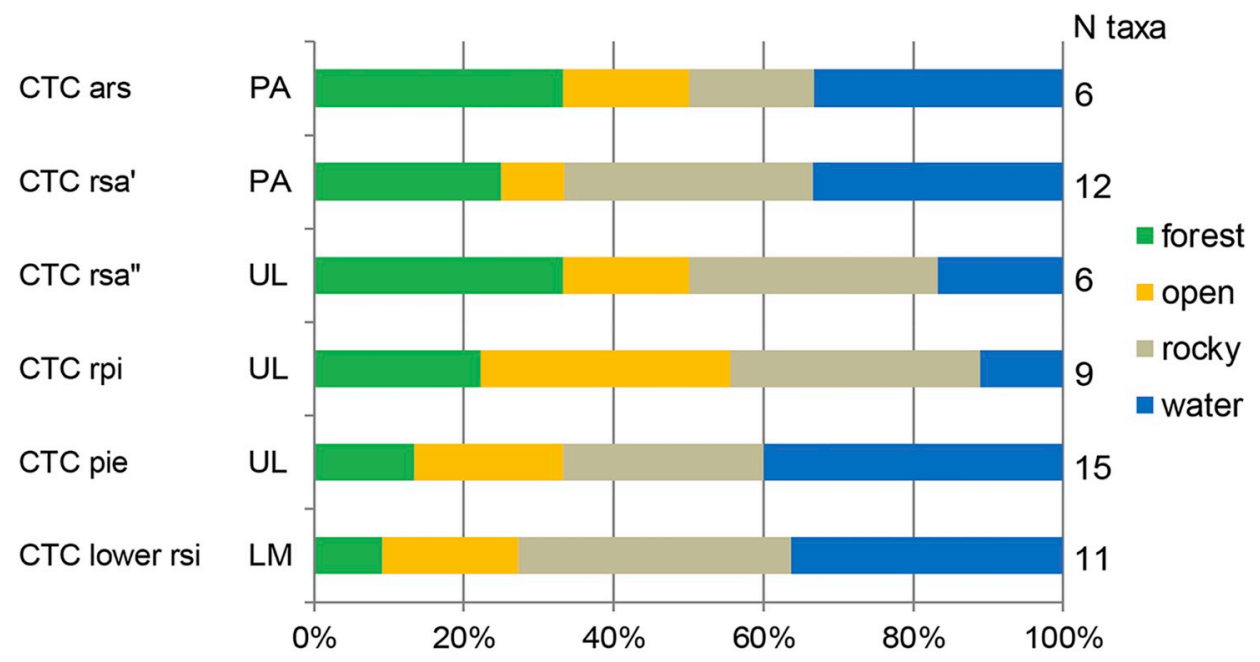

Fig. 8. \% share of the bird taxa of different environments (see legend) in the various layers of Grotta di Castelcivita, calculated on the total number of bird taxa of each layer. $\mathrm{LM}=$ Late Mousterian; UL = Uluzzian; PA = Protoaurignacian. 


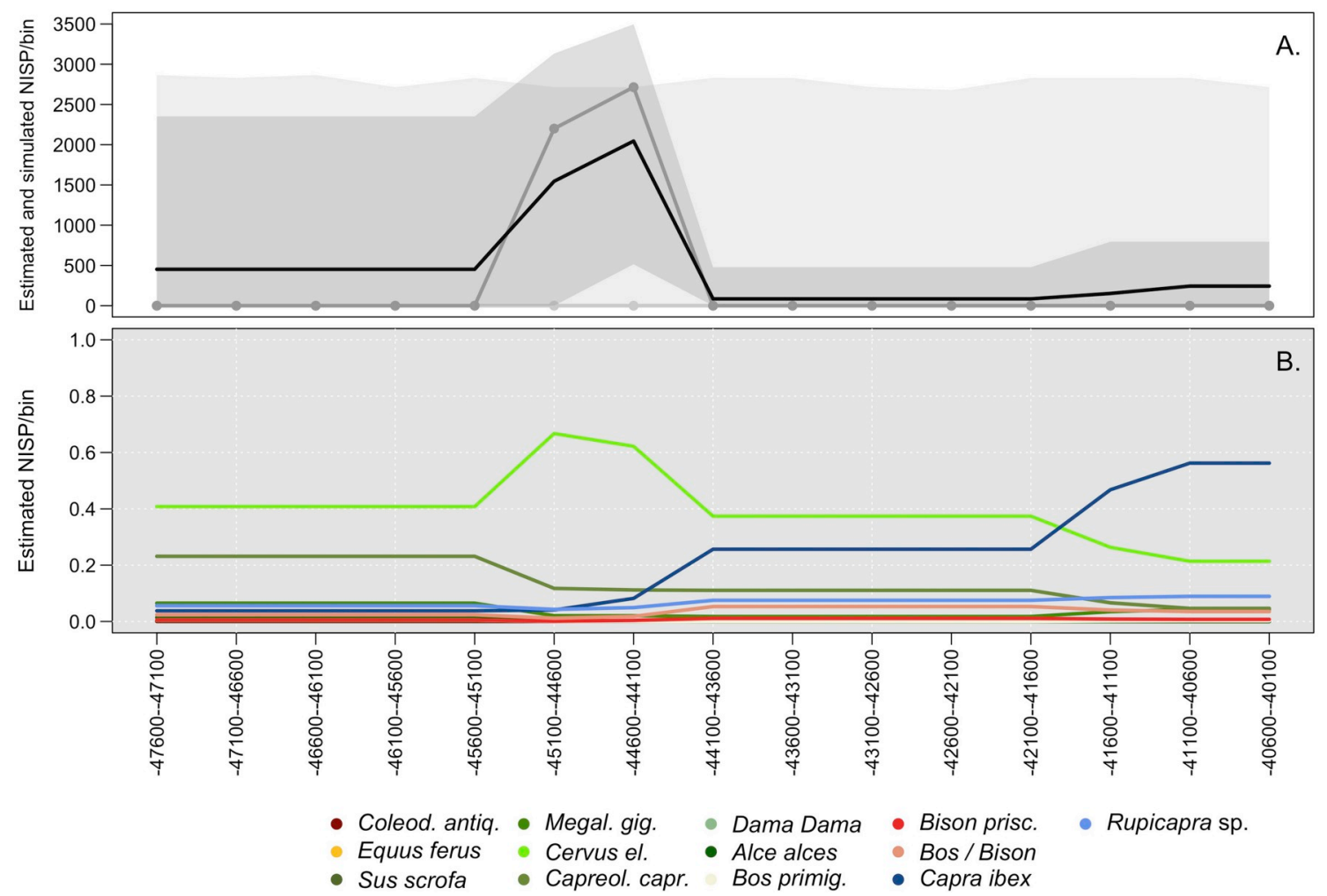

Fig. 9. A) Aoristic sum and estimated chronological frequency distribution of ungulates at Grotta di Fumane. The solid black line represents the aoristic sum, while the darker grey polygon indicates the simulated $95 \%$ confidence interval. The dark grey solid line-and-dots corresponds to simulated median values. The lighter grey envelope is instead the $95 \%$ confidence region of the null model based on uniform frequency distribution across the entire study period; B) Estimated diachronic relative frequency of each ungulate taxon based on aoristic sums computed for 500-year temporal bins. Horizontal axis indicates dates cal. BP.

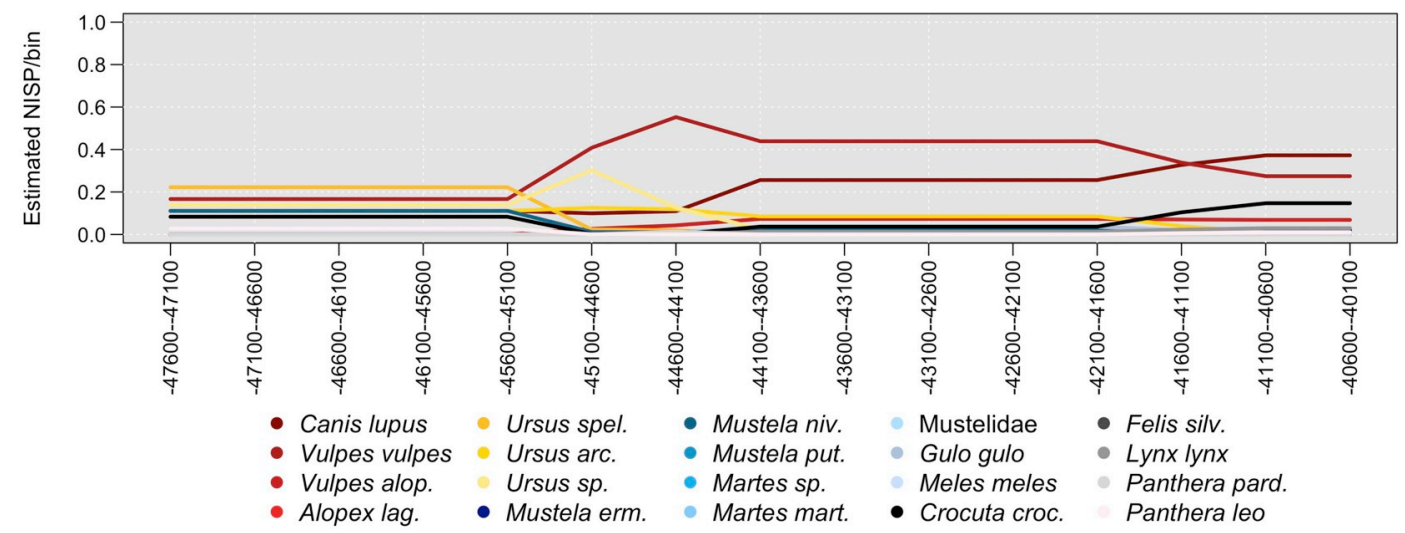

Fig. 10. Estimated diachronic relative frequency of each carnivore taxon at Grotta di Fumane, based on aoristic sums computed for 500-year temporal bins. Horizontal axis indicates dates cal. BP.

in the assemblages. A second moment of more intense process could be identified between 41 and $39 \mathrm{ky}$, corresponding to higher percentages of Capra ibex and Rupicapra rupicapra. These trends confirm what emerged through the inspection of relative frequency estimates and of relative taxonomic abundance analysis, and hint at a potential change in environmental and climatic conditions in the region, but also point to a possible change in hunting and subistence strategies, in particular by comparing ungulates, carnivores, and small preys from taxon abundance analysis.

\subsection{Human exploitation of mammals}

The vast majority of faunal remains uncovered in Northeastern Italy (up to $97 \%$ in some contexts) is highly fragmented, i.e. with length comprised between 0.1 and $3 \mathrm{~cm}$. Intense fragmentation in Northeastern contexts may be imputed to the marrow extraction activity and the use of fire, also to trampling and a set of natural processes (Table 2 and A.13), but the potential influence of hyenas and other carnivores in specific layers (e.g. Grotta di Fumane USS A9-Mousterian, A3-Uluzzian, A2-Protoaurignacian; Table A.12) cannot be excluded, although, once estimated, it appears to be negligible. With the exception of Grotta del Rio Secco, burnt materials contribute to more than $50 \%$ of the total assemblage only in a few Late Mousterian contexts (Table 2). The proportion of calcined bones is higher in Uluzzian layers than in Late Mousterian ones. The frequency of butchering cut marks is also higher in the same temporal interval, while the frequency of percussion marks is lower across the MP-UP transition (Table 3). In the same timeframe, most contexts exhibit higher numbers of cranial bones 


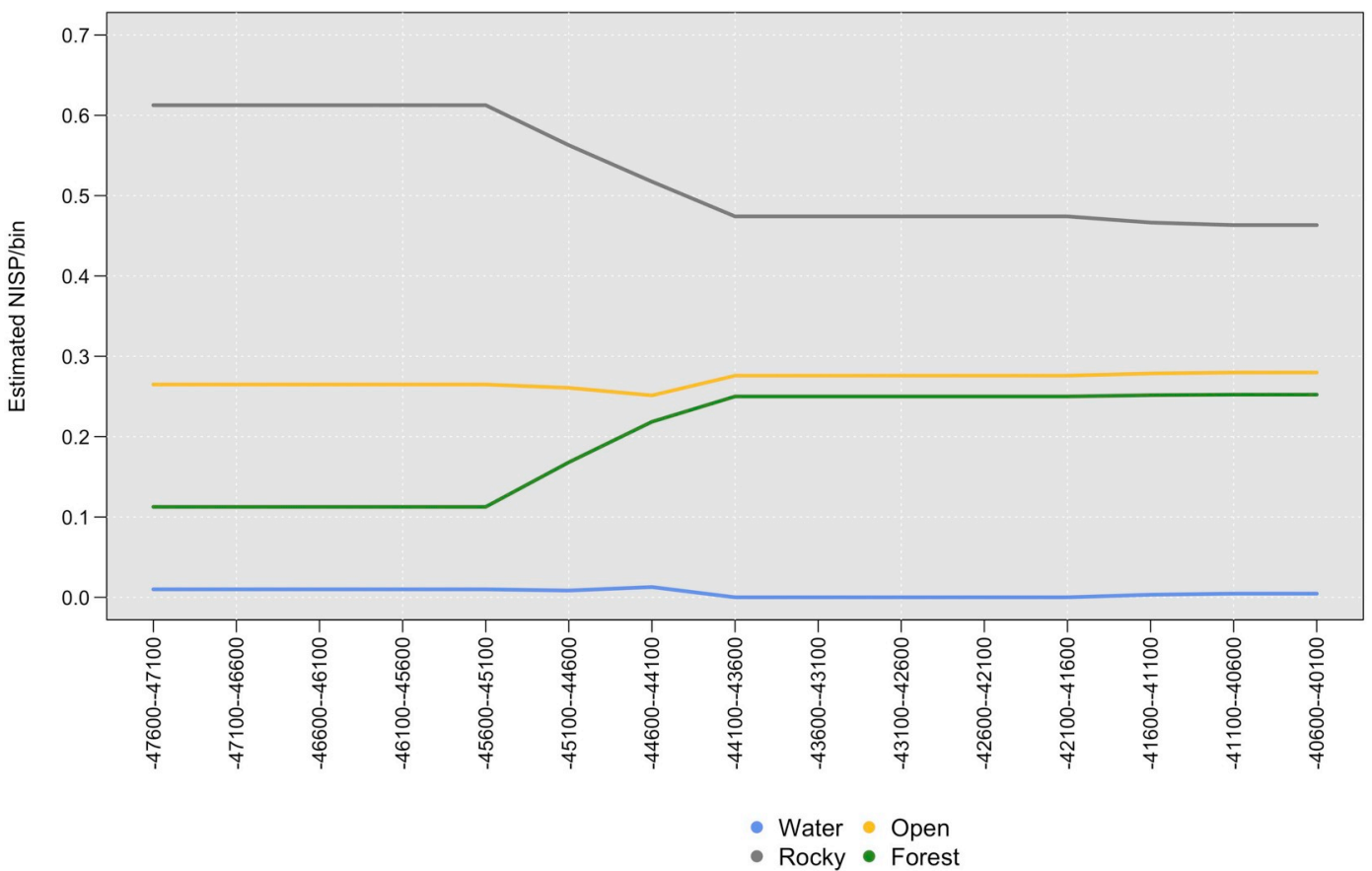

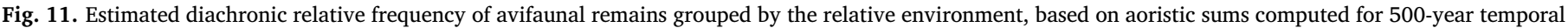
bins. Horizontal axis indicates dates cal. BP.

and limb extremities, and lower frequency of long bone fragments (Table 4). In contrast, elements of the trunk are underrepresented in the whole sequence on all contexts (Table 4).

All ungulate species bear traces of human exploitation (Fig. 13), and anthropic modifications were aimed at obtaining skin, meat, and marrow. The density of wear traces is higher on tibias, femurs and metapodials, radii, and humeri from cervids (red deer, roe deer, and very large specimens of giant deer or moose), and to a lesser extent on the same portions of other ungulates, many of which were likely used as retouchers (Jéquier et al., 2018; Romandini et al., 2018a).

At the end of the Middle Palaeolithic (Fumane A6, A5+ A6; San Bernardino, Unit II), Ursidae (Ursus spelaeus and Ursus arctos) were heavily exploited for fur, meat and marrow, while red fox and beaver were hunted for their skins (Fig. 13). In the Uluzzian at Fumane (A3) there is clear evidence of skinning of foxes, Canis lupus, and Ursus arctos. (Tagliacozzo et al., 2013; Romandini, 2012; Romandini et al., 2014a, 2016a, 2018a, b). At the same site, the Protoaurignacian (A2) shows evidence of anthropic exploitation of all these carnivores (Fig. 13) with the addition of Eurasian lynx (Lynx lynx), while until now there is no evidence of exploitation of avifauna outside of the Mousterian and Uluzzian deposits (Peresani et al., 2011a; Tagliacozzo et al., 2013; Romandini et al., 2014b, 2016b; Fiore et al., 2004, 2016).

The southern Italian assemblages show the same high proportion of ungulates bearing traces of human exploitation as they do in northern Italy (Fig. 13). In addition, also in southern Italian contexts, the spectrum of hunted species mirrors coeval changes in climate and environment, and anthropic modifications are aimed at obtaining skins, meat, and marrow. It is interesting to note the low number of butchered small carnivores and lagomorph taxa, which are particularly concentrated in the Uluzzian and Protoaurignacian phases (Fig. 13). Grotta della Cala in southwestern/Tyrrhenian Italy exhibits increasingly higher percentages of phalanges and sesamoids across the Uluzzian, Protoaurignacian, Early Gravettian and Evolved Gravettian (Benini et al., 1997; Boscato et al., 1997; Boscato and Crezzini, 2006, 2012).

Taphonomic analysis was carried out on a sample of identified ungulate remains from the Late Mousterian (NISP $=67$ ), Uluzzian (NISP $=251$ ), and Protoaurignacian (NISP $=38$ ) layers at Castelcivita (Southwestern Italy; Table 6). Specimens mostly consist of cranial and limb bones (especially metacarpals and metatarsals), while evidence of vertebral bones is limited. The frequency of small limb bones (phalanges, sesamoids, carpal, and tarsal) is higher in Uluzzian and Protoaurignacian layers than in Mousterian ones (Mousterian $=17.9 \%$, Uluzzian $=20.3 \%$, Protoaurignacian $=25 \%$ ). The ratio of diaphysis/ epiphysis fragments is lower in the Uluzzian and Protoaurignacian (4) than in the Late Mousterian (5.3). Anthropic marks were identified on $7.5 \%$ of remains in the Late Mousterian sample, on $10.6 \%$ of Uluzzian material, and have not been identified in the Protoaurignacian assemblage. Carnivore gnawing marks are fewer in the Protoaurignacian layers $(2.6 \%$ of total material) and more abundant in the Late Mousterian (4.5\%) and Uluzzian (7.7\%) layers.

A sample of unidentified remains from the Mousterian levels of Castelcivita $(n=1920)$ is highly fragmented $(91.9 \%$ falls in the class 1-3 cm) (Table 5). In this context diaphysis fragments are the most abundant (40.4\%), followed by spongy bones (16\%), rib fragments (14.7\%), and epiphysis fragments (7.2\%) (Tables A.10 and A.11). Anthropic marks are present on $2.8 \%$ of the unidentified material, while carnivores left traces on $1.5 \%$ of the remains (mostly vertebrae and epiphysis fragments).

As far as skeletal components of the most represented taxa are concerned (Table 7), results obtained at Castelcivita are consistent with what emerged from other southern contexts (Boscato and Crezzini, 2006, 2012), i.e. small limb bones (phalanges, sesamoids, carpals, and tarsals) and epiphyses are present with increasingly higher frequency across the archaeological sequence, while diaphysis fragments exhibit increasingly lower frequency (Tables 7, A.10 and A.11). Nevertheless, the estimate of the contribution of anthropic actions to the formation of faunal assemblages found at Castelcivita may be biased by the presence of spotted hyena (Crocuta crocuta spelaea).

Most unidentified specimens fall in the smallest dimensional category $(1-3 \mathrm{~cm})$, while the percentage of larger findings is higher in Uluzzian deposits (Table 5). Turning to Southeastern Italy, fragments of long bone diaphyses are abundant in the Mousterian assemblages of Riparo l'Oscurusciuto and Grotta del Cavallo whereas epiphysis fragments are rare. At Grotta del Cavallo, on the other hand, percentages of diaphysis fragments are lower in the Uluzzian than they are in the Late Mousterian ones (Table A.11; Boscato and Crezzini, 2006, 2012). 

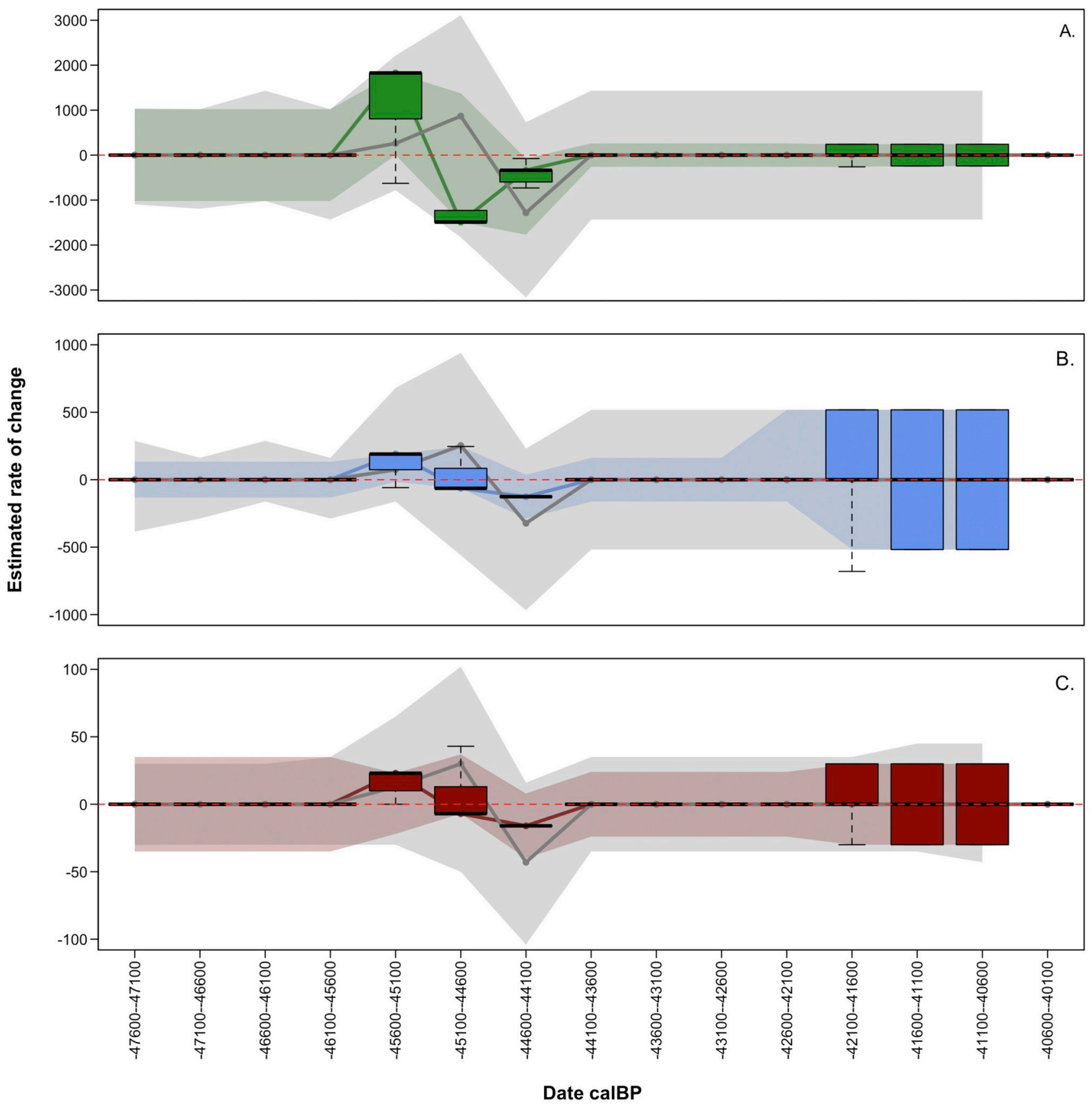

Fig. 12. Simulated diachronic rates of change computed for ungulate families at Grotta di Fumane. Box-plots and coloured polygons indicate the distribution of simulated rates of change based on observed family frequencies, while grey polygons represent $95 \%$ confidence regions for a null model based on the aoristic sums of carnivores uncovered at the same archaeological site. A (green): Cervidae; B (blue): Caprinae; C (red): Bovinae. Based on 5000 simulation runs and computed at 500year bin resolution. (For interpretation of the references to color in this figure legend, the reader is referred to the Web version of this article.)

Considering Riparo Oscurusciuto and Grotta del Cavallo (where bone assemblages are not biased by the action of carnivores), the observed differences between the Late Mousterian and the Uluzzian in the proportion of diaphysis, spongy-bone and phalanges are statistically significant (Tables A.14, A.15, A.16 and A.17). As far as the degree of fragmentation is concerned, it is important not to directly compare any of the (preliminary) values currently available for Southern assemblages with those presented for the northern regions.

\section{Discussion}

\subsection{Comparison of taxon frequencies in macro-mammals between Northeastern, Southwestern, and Southeastern Italy}

Mammal assemblages show that the Middle to Upper Palaeolithic Transition in Northern Italy was associated with a shift to colder and arid climatic conditions, as previously observed by Fiore et al. (2004) and Holt et al. (2019). In Northeastern Italy, human groups used rock shelters in the prealpine fringe and in the alpine foreland and exploited closed forest environments. The surroundings of such shelters were characterised by open environments, alpine meadows and cliffs populated by herbaceous and shrubby species, while humans had to share 

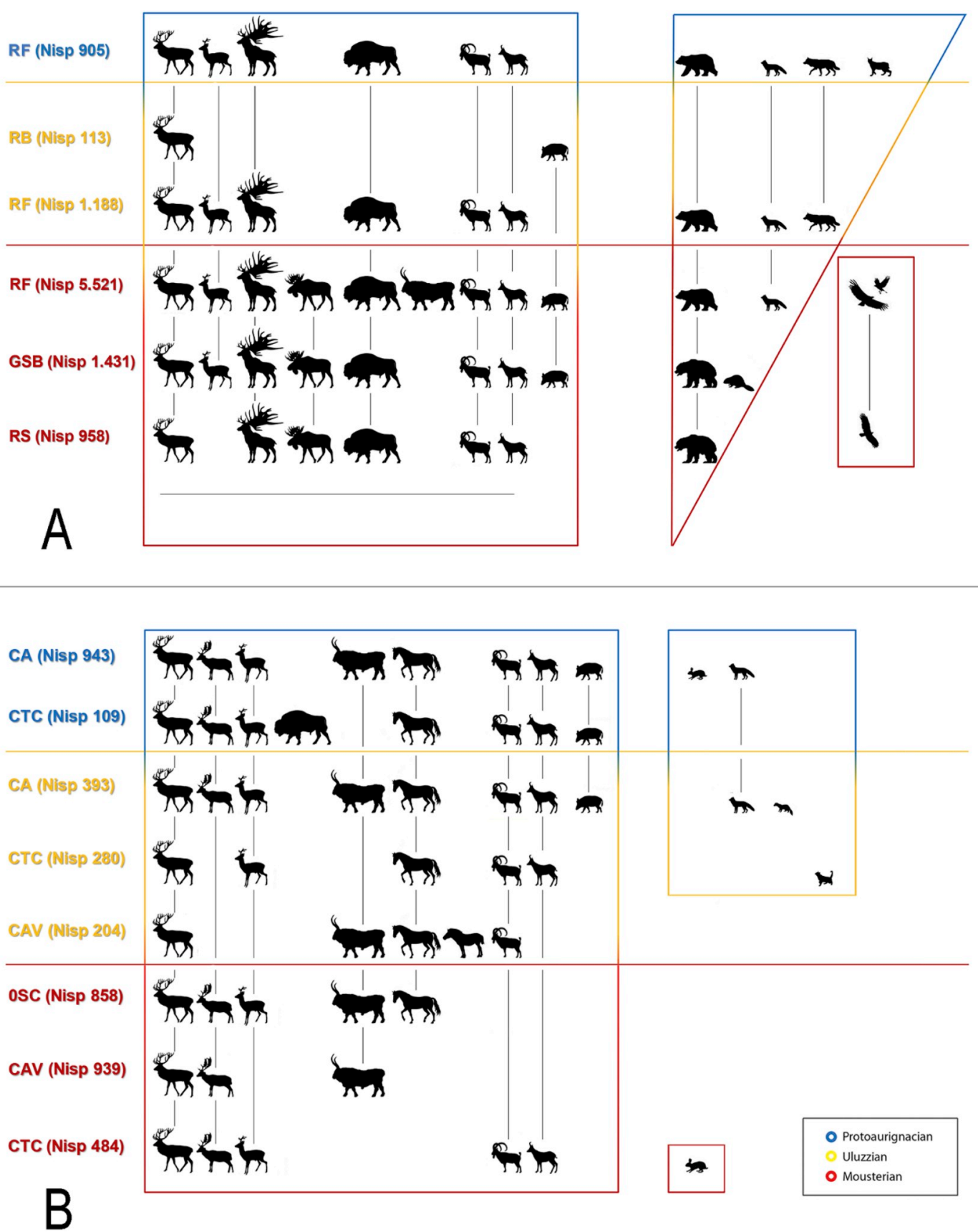

Fig. 13. Comparative summary of diachronic trends concerning the presence of taxa whose bones bear cut-marks, percussion marks and/or evidence of combustion. A) Northern Italy. RF = Grotta di Fumane; RB = Riparo del Broion; GSB = Grotta di San Bernardino; RS = Grotta del Rio Secco. B) Southern Italy. CAV = Grotta del Cavallo; OSC = Riparo l'Oscurusciuto; $\mathrm{CTC}=$ Grotta di Castelcivita; $\mathrm{CA}=$ Grotta della Cala. Colors of temporal phases: Protoaurignacian = blue; Uluzzian = yellow; Late Mousterian $=$ red (for interpreting the color coding in the figure legend the reader is referred to the Web version of this article).

and compete for their shelters with bears (Romandini et al., 2018a). At the end of Middle Palaeolithic, the examined faunal assemblages are dominated by cervidae while species adapted to open environments became considerably less abundant, suggesting a gradual change towards more temperate-humid climate which favoured the expansion of forests and wooded environments before the Uluzzian (such as in Fumane A4). Uluzzian and Protoaurignacian (e.g. Fumane A2) layers bear instead evidence of an abrupt shift to colder and arid conditions, which favoured the diffusion of steppic environments and alpine meadows. These observations are supported by the relative frequencies of cervids and caprids, both of which appear in higher percentages in moments of higher absolute intensity of deposition of ungulate remains. Caprids and bovids also show instances of increase and decrease that are not entirely predicted by the null model based on the distribution of carnivores (i.e. might actually be related to change in environmental conditions).

The aoristic analysis of Grotta di Fumane's zooarchaeological data confirm some of the trends observed by investigating taxon frequency across different sites of Northeastern Italy, especially in the first half of the study sequence. In addition, the comparison of simulated trends against null models based on constant deposition and on the aoristic sum of carnivores provides a means to more formally assess empirical patterns against explicit scenarios. This is particularly useful in a case study affected by small sample size and limited data comparability such as the present one. Most trends appear flattened in the aoristic sum graph, since the analysis explicitly incorporates the temporal uncertainty embedded in the present dataset. Nevertheless, the adoption of this approach paves the 


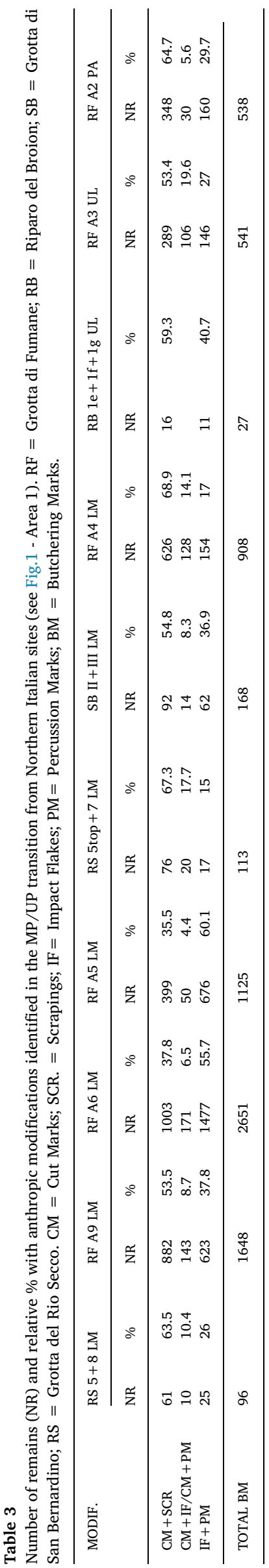

ground for future direct comparisons between northern and southern contexts by highlighting long-term processes that can be directly compared against palaeoecological and palaeoclimatic data collected from a variety of archives, so that future inferences on change in adaptive strategies can be more objective. Additional dates and more detailed information on taphonomy and post-depositional processes will also help shed light on the mechanisms actually underlying the potential diachronic change for Protoaurignacian contexts.

In southern Italy, Late Mousterian deposits exhibit evidence of generally temperate conditions. In this phase, cervids are the most common ungulates in the Tyrrhenian region, while Bos primigenius is the most represented species in Ionian contexts. At Grotta di Castelcivita, this phase is characterised by the same palaeoenvironmental trend documented at Fumane.

The Uluzzian phases at Grotta del Cavallo and Grotta di Castelcivita show data compatible with the establishment of a colder climate, while human groups active at Grotta della Cala experienced more temperate conditions. During MIS 3, the Tyrrhenian side appears to be still characterised by temperate and humid conditions favouring forests and wooded environments, while the Ionian areas were marked by steppic environments and wooded steppe.

Outside of the Italian Peninsula, the only possible comparisons in terms of temporal span and of an archaeological sequence comprising Late Mousterian - Uluzzian - Protoaurignacian (only Aurignacian in Greece) It's represented by Kephalari and Klissoura Cave, Peloponnese, Greece (Starkovich, 2012; Starkovich and Ntinou, 2017; Starkovich et al., 2018). Especially at the latter site, the MIS 3 was highly variable, as suggested by evidence of variation between forested environments, mixed forest-steppe (with red deer, roe deer, chamois and ibex), and drier intervals with steppe species (such as European wild ass, aurochs, ibex and great bustard). The Uluzzian (V) and Aurignacian (IV) layers yielded evidence of fallow deer and small game, in addition to species adapted to both open and forested environments. Plants indicate a mixture of forest and steppe, although taxonomic evenness suggests that conditions were slightly wetter in the Uluzzian layers than during the final MP occupations.

If we exclude Upper Palaeolithic layers of both Kephalari and Klissoura Cave, the exploitation of small game across the transition between Middle and Upper Palaeolithic could be linked to coeval environmental change and a change in resource availability, as it is suggested by the remains of tortoise and hare identified at Klissoura Cave1 in assemblages associated with Neandertals (Starkovich, 2012, 2017, Starkovich et al., 2018). The range of hunted taxa in this region therefore seems to be stable across the Middle and Upper Palaeolithic, and trends can be ascribed to species availability dictated by environmental and climatic change, rather than to convergence in hunting strategies with the Italian Peninsula (Starkovich et al., 2018; Stiner and Munro, 2011). At present a more detailed comparison between the exploitation of animal remains documented in Italy and Greece is not yet possible as research on the subject is still in progress and additional data are required. Nevertheless, trends emerging from taxon abundance analysis are broadly comparable to those identified for Southwestern and Southeastern Italy and documented in the present work. On the other hand, preliminary results presented here suggest in Middle to UP transition a more intensive exploitation of small game in Northeastern Italy than in Southern contexts and the Peloponnese. This finding might be particularly relevant for interpreting regional patterns of change in subsistence/adaptive strategies, considering that large game is generally considered a higher-rank resource than small game.

\subsection{Comparison of avifaunal remains between Fumane and Castelcivita}

The avifaunal assemblages of Grotta di Fumane and Grotta di Castelcivita provide relevant insights on the paleoenvironmental and palaeoclimatic framework of both deposits. The surroundings of Fumane were characterised by mixed and conifer forests, grasslands and alpine meadows with rocky outcrops, cliffs and slow-flowing water 


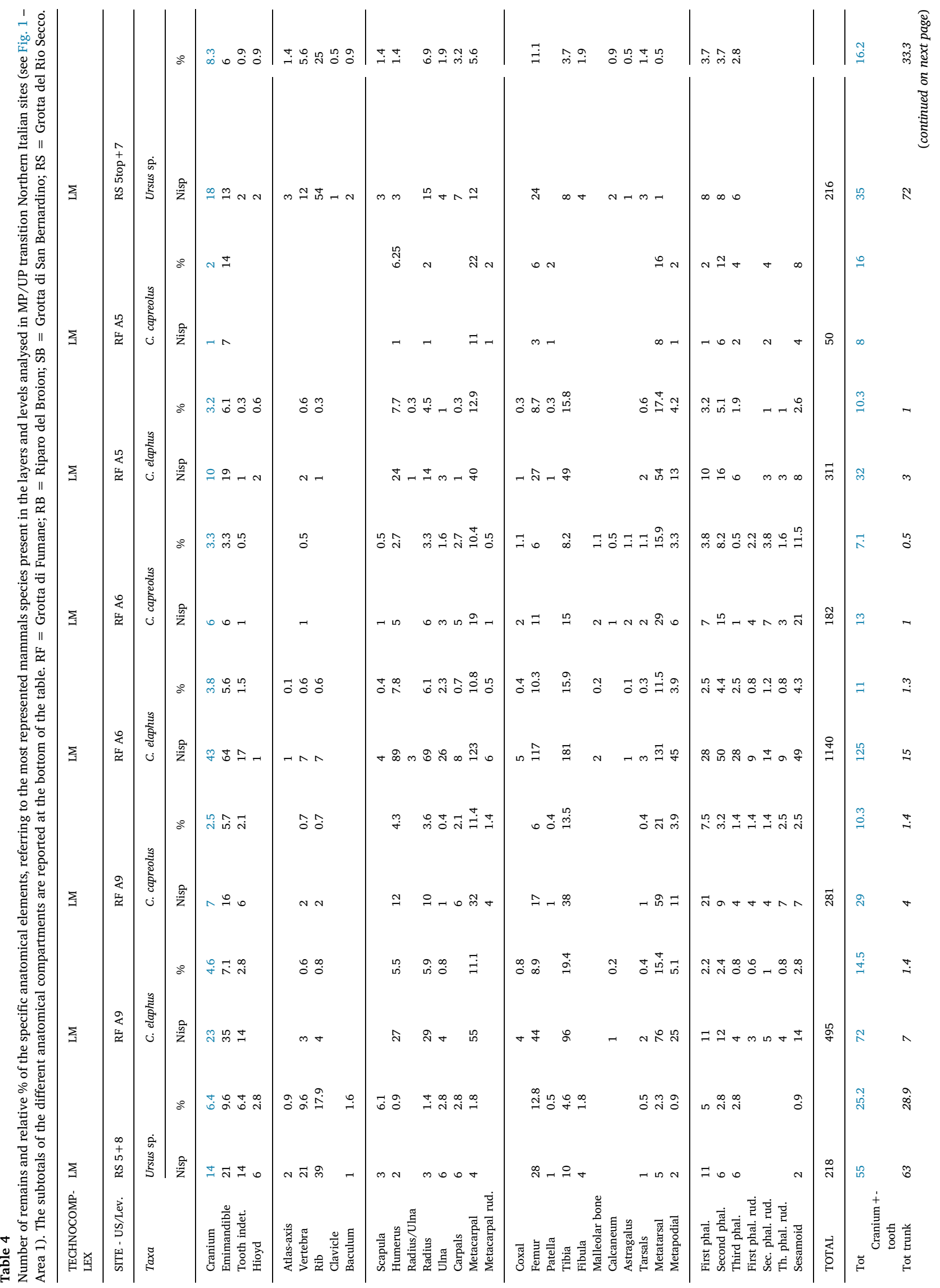




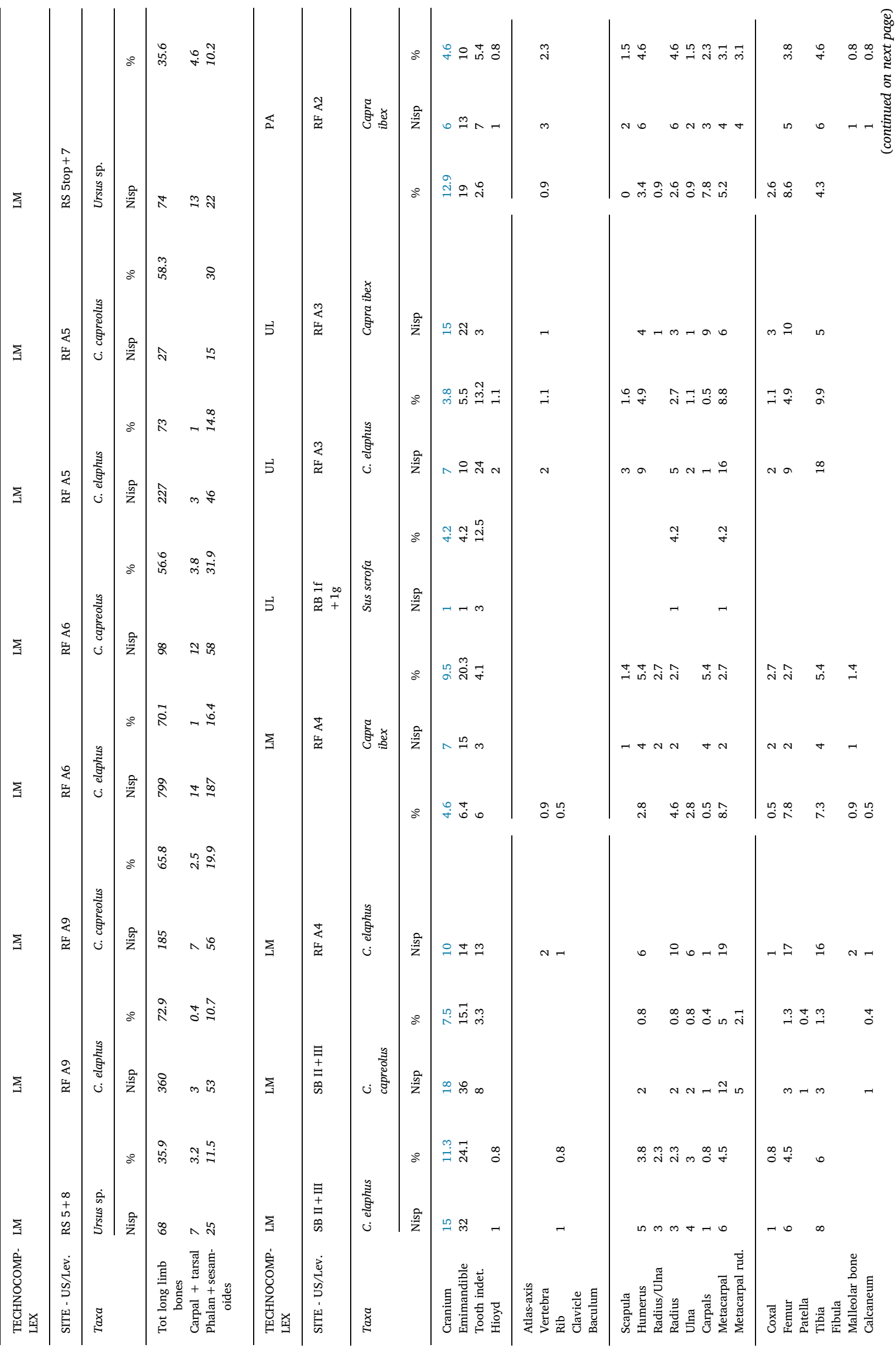




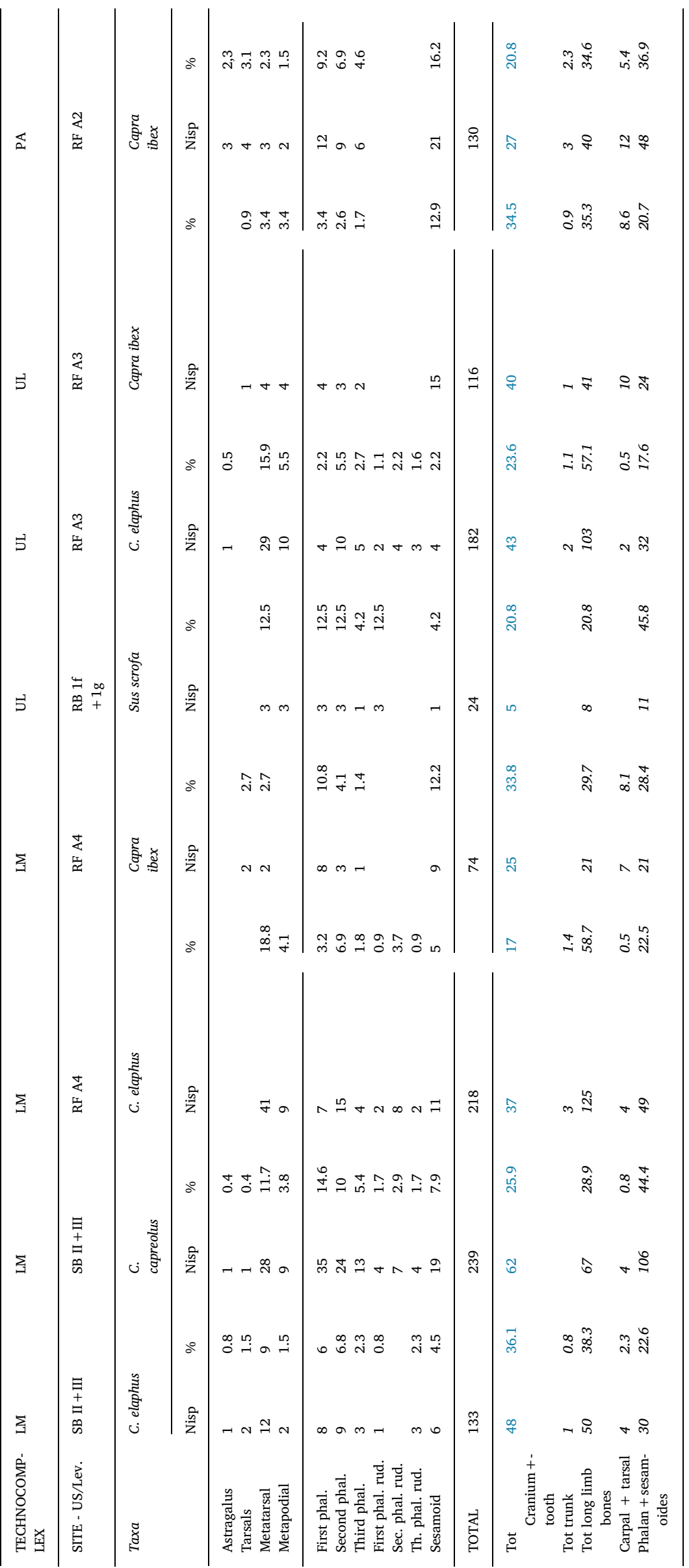


Table 5

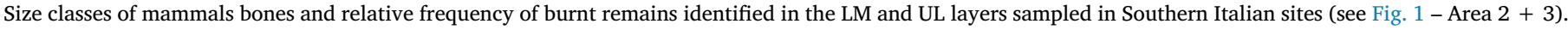
CAV = Grotta del Cavallo; CTC = Grotta di Castelcivita; OSC = Grotta l'Oscurusciuto.

\begin{tabular}{|c|c|c|c|c|c|c|c|c|}
\hline $\begin{array}{l}\text { SITE } \\
\text { US - Levels }\end{array}$ & Technocomplex & $1-3 \mathrm{~cm}$ & $\%$ & $>3 \mathrm{~cm}$ & $\%$ & TOTAL Rem. & Burn. + Calc. & $\%$ \\
\hline CAV EIII & UL & 4201 & 79.9 & 984 & 20.1 & 5185 & 3452 & 82.2 \\
\hline CTC LM & LM & 1764 & 91.9 & 156 & 8.1 & 1920 & Not avail. & Not avail. \\
\hline CAV FII & LM & 9836 & 87.7 & 1378 & 12.3 & 11214 & 1744 & 17.7 \\
\hline OSC US $4 / 1$ & LM & 17472 & 97.4 & 449 & 2.6 & 17921 & 12137 & 67.7 \\
\hline
\end{tabular}

Table 6

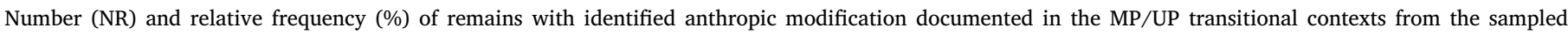

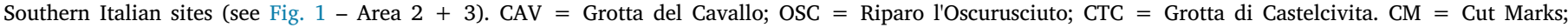
SCR. = Scrapings; IF = Impact Flakes; PM= Percussion Marks; BM = Butchering Marks.

\begin{tabular}{|c|c|c|c|c|c|c|c|c|c|c|c|c|}
\hline \multirow[t]{2}{*}{ MODIF. } & \multicolumn{2}{|c|}{ CTC LM } & \multicolumn{2}{|c|}{ CAV FII LM } & \multicolumn{2}{|c|}{ OSC US 4 LM } & \multicolumn{2}{|c|}{ CAV EII5 UL } & \multicolumn{2}{|c|}{ CTC UL } & \multicolumn{2}{|c|}{ CTC PA } \\
\hline & NR & $\%$ & NR & $\%$ & NR & $\%$ & NR & $\%$ & NR & $\%$ & NR & $\%$ \\
\hline $\mathrm{CM}+\mathrm{SCR}$ & 9 & 0.4 & 63 & 67 & 1 & 3.3 & 75 & 80.6 & 16 & 89 & 5 & 17.2 \\
\hline $\mathrm{CM}+\mathrm{IF} / \mathrm{CM}+\mathrm{PM}$ & 43 & 45.3 & 13 & 13.8 & 26 & 83.9 & 11 & 11.8 & 1 & 5.5 & 12 & 41.4 \\
\hline $\mathrm{IF}+\mathrm{PM}$ & 43 & 45.3 & 18 & 19.2 & 4 & 2.8 & 7 & 0.6 & 1 & 5.5 & 12 & 41.4 \\
\hline TOTAL BM & 95 & & 94 & & 31 & & 93 & & 18 & & 29 & \\
\hline
\end{tabular}

bodies. The environmental framework of Grotta di Castelcivita was instead characterised by wetlands in the plains in front of the cave and by drier habitats like grasslands, rocky terrains and rock walls, alternated to conifer or mixed forests at higher altitudes.

Bird taxa across Italy indicate the presence of a consistently colder climate than the present one. Nevertheless, in the southwestern/ Tyrrhenian area (Fig. 1, Area 2) climate seems milder and more temperate than in the Adriatic area, as suggested by the absence of boreal bird species and by a lower proportion of high altitude bird taxa in the former. Once again, faunal assemblages mirror climatic differences between Tyrrhenian and Ionian/Adriatic regions mostly due to the effect of the Balkanic influence on the latter. The Middle Paleolithic assemblages from both sites provide evidence of temperate-cool climate, where the species of open and rocky habitats prevail. The Late Mousterian Layer A6 at Fumane hints at a possible cold oscillation, however, and the Uluzzian at both sites (A3 at Grotta di Fumane, and CTC rsi at Grotta di Castelcivita) shows higher percentages of bird taxa typical of open habitats possibly due to colder conditions linked to Heinrich Event 4 (Higham et al., 2009; Moroni et al., 2018; Lopéz-García et al., 2015). Protoaurignacian deposits provide evidence for the persistence of harsh conditions which characterized previous phases (Cassoli and Tagliacozzo, 1994a). In spite of the low NISP, one exception seems to be represented by the latest Protoaurignacian layers of Castelcivita (gic-ars; referring here particularly to the layer ars, as the bird bone sample of the layer gic was too small to include it in the analysis), that yielded evidence for climatic shift toward more humid conditions (Cassoli and Tagliacozzo, 1997; Gala et al., 2018).

\subsection{Taphonomy}

Despite the facts that taphonomic data are still only partially investigated in most of the sampled contexts and that the majority of identified patterns cannot be proven to be statistically significant because of small sample size, interesting preliminary trends emerge. Although future studies may alter the pattern detected so far, at present, the percentage of calcined remains during the Uluzzian and Protoaurignacian levels in Northeastern Italian contexts is higher than the frequency of the same items in previous phases, and hints at a possible behavioural change linked to the use of fire: greater intensity and duration of use of the hearths, differentiation of fuel and/or cooking of animal resources. Cut-marks are also more frequent across the transition, while the degree of bone fragmentation for marrow extraction is higher in Mousterian layers than in later deposits. In the Early Upper Palaeolithic overall (i.e., Uluzzian and Protoaurignacian) there are higher percentages of cranial bones and limb extremities, with a consequent lower proportion of long bones. This trend may be imputed partly to human selection and partly to the use of the cave by hyenas and other carnivores. The remains of the most frequently hunted large (Cervidae, Bovinae) and medium-sized (Caprinae) ungulates show cut- and percussion-marks, all of which point to skinning, butchering, and marrow extraction. Over the same timespan, bears and middle- and small-sized carnivores appear to be more frequently exploited, suggesting a broadening in the range of species hunted for skin and fur (Collard et al., 2016).

Avifaunal assemblages provide evidence of human consumption of birds and contribute to an understanding of the role of avifaunal resources in the subsistence strategies of Middle Palaeolithic hominins (Peresani et al., 2011a; Romandini, 2012; Tagliacozzo et al., 2013; Fiore et al in this issue; Romandini et al., 2014b, 2016a, b; Gala et al., 2018; Fiore et al., 2004, 2016). The exploitation of these resources is testified by recognizable taphonomic indicators such as evidence for the exploitation of feathers from various raptors and other birds.

In the same way, evidence of Neandertal reliance on small mammal prey increased over the past 10 years due to the reassessment of faunal assemblages from a new taphonomic perspective (Romandini et al., 2018b; Morin et al., 2019).

In Ionian contexts, Late Mousterian assemblages exhibit a lack (or at least a scarcity) of long-bone epiphyses, carpal and tarsal bones, phalanges and sesamoides. In the analysed Late Mousterian samples from Grotta del Cavallo and Riparo l'Oscurusciuto, this evidence cannot be attributed to carnivores, differential bone density and other post-depositional processes (Boscato and Crezzini, 2006, 2012). The frequency of different anatomical parts (\% of Minimum Animal Units, Binford, 1984) of Bos primigenius and the modal species in US 4 at Riparo l'Oscurusciuto were compared against Emerson's utility indices related to present-day Bison bison (Emerson, 1990, 1993). Previous results suggest a relationship between bone frequency and their content in marrow and fat, which was probably crucial in 
Table 7

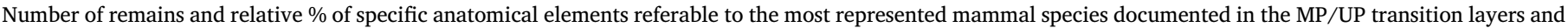

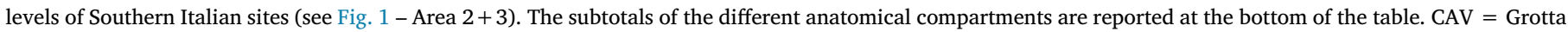
del Cavallo; OSC = Riparo l'Oscurusciuto; CTC = Grotta di Castelcivita; CALA = Grotta della Cala.

\begin{tabular}{|c|c|c|c|c|c|c|c|c|c|c|c|c|c|c|c|c|}
\hline TECHNOCOMPLEX & LM & & LM & & LM & & UL & & UL & & UL & & $\mathrm{PA}$ & & $\mathrm{PA}$ & \\
\hline SITE - US/Lev. & OSC & & CTC & & $\begin{array}{l}\text { CAV (all } \\
\text { lev.) }\end{array}$ & & CAV EIII5 & & CTC & & CALA 14 & & CTC ars-gic & & $\begin{array}{l}\text { CALA 13- } \\
10\end{array}$ & \\
\hline \multirow[t]{2}{*}{ TAXA } & Bos primig. & & $\begin{array}{l}\text { Dama } \\
\text { dama }\end{array}$ & & Bos primig. & & Bos primig. & & C. elaphus & & $\begin{array}{l}\text { Dama } \\
\text { dama }\end{array}$ & & C. elaphus & & C. elaphus & \\
\hline & Nisp & $\%$ & Nisp & $\%$ & Nisp & $\%$ & Nisp & $\%$ & Nisp & $\%$ & Nisp & $\%$ & Nisp & $\%$ & Nisp & $\%$ \\
\hline Antler/Horn & & & & & & & & & 3 & 3.2 & & & & & 1 & 0.2 \\
\hline Cranium & 13 & 2.6 & 4 & 2.8 & 3 & 0.8 & 2 & 2.3 & 1 & 1 & 5 & 3.4 & & & 17 & 3.5 \\
\hline Emimandible & 43 & 8.7 & 9 & 6.3 & 12 & 3 & & & 4 & 4.4 & 13 & 8.7 & 1 & 4.3 & 42 & 8.8 \\
\hline Decidous teeth & 8 & 1.6 & 9 & 6.3 & 49 & 12.4 & 1 & 1.2 & 2 & 2.1 & & & 2 & 8.7 & & \\
\hline Permanent teeth & 202 & 40.6 & 60 & 41.7 & 201 & 51 & 10 & 11.6 & 7 & 7.5 & & & 8 & 34.8 & & \\
\hline Perm. + Deciduos teeth & 11 & 2.2 & & & & & & & & & 46 & 30.9 & & & 133 & 27.8 \\
\hline Tooth indet. & 47 & 9.5 & 10 & 6.9 & 21 & 5.3 & 5 & 5.8 & & & 2 & 1.3 & 2 & 8.7 & 7 & 1.5 \\
\hline Hioyd & & & & & 7 & 1.8 & 1 & 1.2 & & & & & & & & \\
\hline $\begin{array}{l}\text { Atlas-axis } \\
\text { Vertebra } \\
\text { Rib } \\
\text { Clavicle }\end{array}$ & & & & & & & & & $\begin{array}{l}3 \\
1\end{array}$ & $\begin{array}{l}3.3 \\
1\end{array}$ & 1 & 0.7 & & & 2 & 0.4 \\
\hline Scapula & & & & & & & & & & & & & & & 1 & 0.2 \\
\hline Humerus & 10 & 2 & 3 & 2.1 & & & 4 & 4.7 & 5 & 5.5 & 2 & 1.3 & & & 9 & 1.9 \\
\hline Radius/Ulna & 5 & 1 & & & & & 2 & 2.3 & 1 & 1 & & & & & & \\
\hline Radius & 10 & 2 & 5 & 3.5 & 9 & 2.3 & 1 & 1.2 & 4 & 4.4 & 6 & 4 & & & 12 & 2.5 \\
\hline Ulna & 4 & 0.8 & & & 4 & 1 & 1 & 1.2 & & & 1 & 0.7 & & & 6 & 1.3 \\
\hline Carpals & & & 2 & 1.4 & & & 4 & 4.7 & 8 & 8.7 & 3 & 2 & & & 15 & 3.1 \\
\hline $\begin{array}{l}\text { Metacarpal } \\
\text { Metacarpal rud. }\end{array}$ & 12 & 2.4 & 3 & 2.1 & 8 & 2 & 2 & 2.3 & 16 & 17.2 & & & 3 & 13 & 38 & 7.9 \\
\hline Coxal & & & & & & & & & & & & & & & 1 & 0.2 \\
\hline Femur & 5 & 1 & 4 & 2.8 & & & & & 4 & 4.4 & & & & & 2 & 0.4 \\
\hline Patella & & & & & & & & & 1 & 1 & 1 & 0.7 & & & 1 & 0.2 \\
\hline $\begin{array}{l}\text { Tibia } \\
\text { Fibula }\end{array}$ & 51 & 10.3 & & & 3 & 0.8 & 3 & 3.5 & 9 & 9.7 & 4 & 2.7 & & & 5 & 1 \\
\hline $\begin{array}{l}\text { Malleolar bone } \\
\text { Calcaneum } \\
\text { Astragalus }\end{array}$ & & & & & & & 1 & 1.2 & & & & & & & 3 & 0.6 \\
\hline Tarsals & 7 & 1.4 & 2 & 1.4 & 6 & 1.5 & 6 & 7 & & & 14 & 9.4 & & & 14 & 2.9 \\
\hline Metatarsal & 38 & 7.6 & 12 & 8.3 & 21 & 5.3 & 5 & 5.8 & 14 & 15 & 22 & 14.7 & 3 & 13.2 & 78 & 16.3 \\
\hline Metapodial & 4 & 0.8 & 3 & 2.1 & 16 & 4.1 & 2 & 2.3 & & & 8 & 5.4 & 2 & 8.7 & 24 & 5 \\
\hline First phal. & 13 & 2.6 & 6 & 4.2 & 15 & 3.8 & 16 & 18.6 & 7 & 7.5 & 12 & 8.1 & 1 & 4.3 & 29 & 6.1 \\
\hline Second phal. & 6 & 1.2 & 9 & 6.3 & 4 & 1 & 8 & 9.3 & 2 & 2.1 & 6 & 4 & 1 & 4.3 & 21 & 4.4 \\
\hline $\begin{array}{l}\text { Third phal. } \\
\text { First phal. rud. } \\
\text { Sec. phal. rud. } \\
\text { Th. phal. rud. }\end{array}$ & 1 & 0.2 & 2 & 1.4 & 1 & 0.3 & 1 & 1.2 & & & & & & & 9 & 1.9 \\
\hline Sesamoid & 7 & 1.5 & 1 & 0.4 & 14 & 3.6 & 11 & 12.6 & 1 & 1 & 3 & 2 & & & 9 & 1.9 \\
\hline TOTAL & 497 & & 144 & & 394 & & 86 & & 93 & & 149 & & 23 & & 479 & \\
\hline Tot Cranium + tooth & 324 & 65.2 & 92 & 63.8 & 293 & 74.4 & 19 & 22.1 & 17 & 18.3 & 66 & 44.3 & 13 & 56.5 & 200 & 41.7 \\
\hline Tot trunk & & & & & & & & & 4 & 4.3 & 1 & 0.7 & & & 4 & 0.9 \\
\hline Tot long limb bones & 146 & 29.4 & 30 & 20.8 & 61 & 15.5 & 21 & 24.4 & 53 & 57 & 44 & 29.5 & 8 & 34.8 & 178 & 37.2 \\
\hline Carpal + tarsal & & & 5 & 3.6 & 6 & 1.5 & 10 & 11.6 & 8 & 8.6 & 17 & 11.4 & & & 29 & 6 \\
\hline Phal. + sesamoides & 27 & 5.4 & 17 & 11.8 & 34 & 8.6 & 36 & 41.9 & 11 & 11.8 & 21 & 14.1 & 2 & 8.7 & 68 & 14.2 \\
\hline
\end{tabular}

the choice to select specific anatomical parts and to carry them back to camps/sites (Boscato and Crezzini, 2006, 2012). Recent studies demonstrated that at least at Riparo l'Oscurusciuto spongy bones were not systematically used as fuel in hearths (Spagnolo et al., 2016), suggesting their possible use as food (Costamagno and Rigaud, 2014). In southern Italy, Upper Palaeolithic assemblages indicate a different manner of exploitating ungulate bones (Boscato and Crezzini, 2006, 2012). A large amount of long-bone epiphyses and spongy elements (including carpal and tarsal bones) were not destroyed and can be found in these assemblages. Unlike Neandertals, who were evidently not interested in phalanges and probably left them at the kill sites, modern humans usually transported these small skeletal parts to their campsites where they fragmented them to recover 
the particular fat they contained: Morin (2006) underlines that, although the phalanges contain a low quantity of marrow, it is qualitatively different than the marrow contained in long bones, due to its higher percentage of oleic acid. These data suggest a change in processing hard animal tissues by Upper Palaeolithic people across southern Italy, a change that is already visible in Uluzzian assemblages, a documented by the case of Layer EIII5 at Grotta del Cavallo (Boscato and Crezzini, 2006, 2012).

\section{Conclusions}

The data collected and analysed show that human adaptive strategies changed over time to cope with variability in local topographic and ecological conditions, as well as with uncertainty in resource availability. Uncertainty and bias are critically embedded in the procurement and exploitation of animal resources, especially in such a fragmented and small-scale scenario as the Italian Peninsula. A sample of key sites from both southern and northern Italy offers evidence of how groups of Neandertals and modern humans occupied the Tyrrhenian and Ionian areas, as well as through the area between the great alluvial plain of the river Po and the Pre-Alpine mountains. In this context, a thorough and detailed zooarchaeological approach offers a unique perspective on palaeoenvironmental and palaeoecological settings, as well as on hunting and subsistence strategies. In the present study, we presented state-of-the-art evidence on the differential presence of large mammals and avifauna across Late Mousterian, Uluzzian, and Protoaurignacian assemblages from Italy. Incorporating an aoristic analysis further allows us to explicitly address the amount of temporal uncertainty embedded in one of the zooarchaeological assemblages of interest. While losing detail on individual archaeological layers, this method offers a practical solution to help overcome the effects of timeaveraging and of the lack of information on layer-specific accumulation rates. At the same time, aoristic sums for ungulates, rates of change based on simulated data, and the comparison with null models depict a conservative scenario useful for inferring instances of absolute increase or decline of given taxa or families over time. The analysis of environmentally-informative bird taxa added significant detail to the environmental trends provided by mammal remains, improving our understanding of the climatic framework of the Middle-Upper Paleolithic transition. The future addition of micromammals to the analysis will make it possible to add detail on local biotopes, and to further test inferences on palaeoclimatic change in the different contexts. Interesting hypotheses on human behavioural ecology also emerge from the examined archaeological assemblages, although additional evidence is still clearly required for objectively test inferences about Uluzzian and Protoaurignacian contexts. In particular, differences seem to emerge in the use of fire (especially in terms of temperatures and bone processing) between Late Mousterian layers and the subsequent phases. More substantial data on the distribution of ungulate limb elements suggest a marked change in prey exploitation between the Late Mousterian and the Early Upper Palaeolithic in southern Italy, while northern sites show that a higher variety of processing techniques was already present in the Late Mousterian. As concerns differences in hunting strategies, traces of an increasing preference for small- and medium-sized mammals (carnivores, rodents, lagomorphs) can be already documented for the transition to Protoaurignacian, although presently available evidence is exclusively qualitative. Future research will ascertain if this difference can be ascribed to a forced expansion of niche breadth due to economic and technological competition between Neandertals and modern humans (Hockett and Haws, 2005).

The above mentioned hypotheses cannot yet be tested because of small sample size in all the analysed classes, and the emerging trends may or may not be confirmed by adding evidence on the same sites as well as on other, currently underrepresented areas of the Italian Peninsula to the analyses presented here. Over the next three years, the project ERC n. 724046 - SUCCESS will build on the results presented here by acquiring novel zooarchaeological and chronological evidence on all the mentioned contexts (Fig. 1), by directly comparing faunal time series to palaeoenvironmental and palaeoclimatic data, and by relying on innovative methods (Pothier Bouchard et al., 2019; Pothier Bouchard et al. in this issue) such as ZooMS (ZooArchaeology by Mass Spectrometry). This evidence will contribute to helping resolve or at least clarify longstanding debates surrounding strategic and technological shifts which occurred during the Middle-Upper Paleolithic transition and will help situate the questions concerning contacts between Neandertals and modern humans in Italy (and the eventual replacement of the former by the latter) in the broader framework of complex adaptive strategies and long-term human-environment interactions.

\section{Data availability}

Datasets, scripts and related commands used to generate all of the results described in the paper are available at (http://doi.org/10.6092/ unibo/amsacta/6209).

\section{Acknowledgments}

This project has received funding from the European Research Council (ERC) under the European Union's Horizon 2020 research and innovation programme (grant agreement No 724046 - SUCCESS); website: http://www.erc-success.eu/.

Research at Fumane is coordinated by the Ferrara University (M.P.) in the framework of a project supported by MIBAC, public institutions (Lessinia Mountain Community, Fumane Municipality and others). Research at Riparo del Broion (M.R.) and Grotta di San Bernardino (M.P.) is designed by Ferrara and Bologna University and was supported by MIBAC, the Province of Vicenza, the Veneto Region, the Italian Ministry of Research and Education, and institutions (Leakey Foundation, Spring 2015 Grant; Fondazione CariVerona). Research at Rio Secco (M.P. and M.R.) is designed by Ferrara University and was supported by MIBAC the Administration of the Clauzetto Municipality and the Friuli Venezia Giulia Region and a group of public institutions (Ecomuseo delle Dolomiti Friulane, "Lis Aganis", BIM Tagliamento Consortium, Pordenone Province), Foundations (Fondazione CRUP) and private companies (Friulovest Banca). Researches at Grotta di Castelcivita, Grotta della Cala and Riparo L'Oscurusciuto are coordinated by the University of Siena (A.R., A.M. and F.B. respectively). We thank the Soprintendenza Archeologia, Belle Arti e Paesaggio per le Province di Brindisi, Lecce e Taranto, and the Soprintendenza Archeologia, Belle Arti e Paesaggio per le Province di Salerno e Avellino for kindly supporting our research and fieldwork in Apulia and Campania over the years. We acknowledge the Municipalities of Camerota, Castelcivita, Ginosa and the Parco Nazionale del Cilento and Vallo di Diano for logistic support. Research at Riparo Bombrini is coordinated by the University of Genoa (F.N.) and Université de Montréal (J.R.S) and supported by FRQSC grant 2016-NP-193048 (J.R.S) and SSHRC Insight Grant 435-2017-1520 (J.R.S. \& F.N.), under the auspices of the Soprintendenza Archeologia, Belle Arti e Paesaggio per la città metropolitana di Genova e le province di Imperia, La Spezia e Savona, with logistical support from the Istituto Internazionale di Studi Liguri (Bordighera), the Museo preistorico nazionale dei Balzi Rossi and the Polo museale della Liguria. 


\section{Appendices}

Table A.1

Total Nisp and relative frequency of Ungulata documented in levels and layers of Northern Italy (Area 1) presented in chronological-cultural order. RF $=$ Grotta di Fumane; RB = Riparo del Broion; SB = Grotta di San Bernardino; RS = Grotta del Rio Secco.

\begin{tabular}{|c|c|c|c|c|c|c|c|c|c|c|c|c|c|c|c|c|c|c|c|c|}
\hline \multirow[t]{2}{*}{ Taxa } & \multicolumn{2}{|c|}{ RS $5+8-\mathrm{LM}$} & \multicolumn{2}{|c|}{ RF A9 - LM } & \multicolumn{2}{|c|}{ RF A6 - LM } & \multicolumn{2}{|c|}{$\begin{array}{l}\text { RF A5/A5+A6 - } \\
\mathrm{LM}\end{array}$} & \multicolumn{2}{|c|}{$\begin{array}{l}\text { RS 5top+7 - } \\
\text { LM }\end{array}$} & \multicolumn{2}{|c|}{ SB II + III - LM } & \multicolumn{2}{|c|}{ RF A4 - LM } & \multicolumn{2}{|c|}{ RB 1f-1g - UL } & \multicolumn{2}{|c|}{ RF A3 - UL } & \multicolumn{2}{|c|}{ RF A2-A2R - PA } \\
\hline & Nisp & $\%$ & Nisp & $\%$ & Nisp & $\%$ & Nisp & $\%$ & Nisp & $\%$ & Nisp & $\%$ & Nisp & $\%$ & Nisp & $\%$ & Nisp & $\%$ & Nisp & $\%$ \\
\hline Stephanorhinus sp. & & & & & & & & & & & 2 & 0.3 & & & & & & & & \\
\hline Coelodonta antiquitatis & & & & & & & & & & & & & & & & & 1 & 0.2 & & \\
\hline Sus scrofa & 2 & 4.8 & 2 & 0.2 & 2 & 0.1 & & & 1 & 1.7 & 36 & 5.2 & & & 21 & 35.6 & & & & \\
\hline Megaloceros giganteus & 5 & 11.9 & 79 & 6.5 & 28 & 1.8 & 10 & 2.1 & 14 & 24.1 & 12 & 1.7 & 12 & 2.5 & 2 & 3.4 & 8 & 1.8 & 34 & 4.3 \\
\hline Cervus elaphus & 7 & 16.7 & 495 & 40.8 & 1095 & 69.7 & 297 & 62 & 6 & 10.3 & 136 & 19.6 & 242 & 50 & 5 & 8.5 & 169 & 37.4 & 170 & 21.4 \\
\hline Capreolus capreolus & 1 & 2.4 & 281 & 23.1 & 182 & 11.6 & 48 & 10 & & & 251 & 36.2 & 54 & 11.2 & 3 & 5.1 & 50 & 11.1 & 37 & 4.7 \\
\hline Alces alces & 2 & 4.8 & 17 & 1.4 & 4 & 0.3 & 1 & 0.2 & 5 & 8.6 & 24 & 3.5 & & & 3 & 5.1 & & & & \\
\hline Cervidae & 6 & 14.3 & 166 & 13.7 & 128 & 8.2 & 39 & 8.1 & 19 & 32.8 & 135 & 19.5 & 29 & 6 & 13 & 22 & 33 & 7.3 & & \\
\hline Bos primigenius & & & 6 & 0.5 & & & & & & & 1 & 0.1 & & & 1 & 1.7 & & & 2 & 0.3 \\
\hline Bison priscus & 1 & 2.4 & 6 & 0.5 & 2 & 0.1 & & & 2 & 3.4 & & & 5 & 1 & 1 & 1.7 & 5 & 1.1 & 6 & 0.8 \\
\hline Bos/Bison & 10 & 23.8 & 29 & 2.4 & 13 & 0.8 & 10 & 2.1 & 8 & 13.8 & 33 & 4.8 & 16 & 3.3 & 1 & 1.7 & 24 & 5.3 & 28 & 3.5 \\
\hline Capra ibex & 5 & 11.9 & 46 & 3.8 & 54 & 3.4 & 30 & 6.3 & 2 & 3.4 & 3 & 0.4 & 82 & 16.9 & 1 & 1.7 & 116 & 25.7 & 447 & 56.2 \\
\hline Rupicapra rupicapra & 3 & 7.1 & 68 & 5.6 & 55 & 3.5 & 32 & 6.7 & & & 53 & 7.6 & 31 & 6.4 & 4 & 6.8 & 34 & 7.5 & 71 & 8.9 \\
\hline Caprinae & & & 19 & 1.6 & 7 & 0.4 & 12 & 2.5 & 1 & 1.7 & 8 & 1.2 & 13 & 2.7 & 4 & 6.8 & 12 & 2.7 & & \\
\hline Total Ungulata & 42 & & 1214 & & 1570 & & 479 & & 58 & & 694 & & 484 & & 59 & & 452 & & 795 & \\
\hline
\end{tabular}

Table A.2

Total Nisp and relative frequency of Carnivora documented in levels and layers of Northern Italy (Area 1 in Fig.1) presented in chronological-cultural order. RF $=$ Grotta di Fumane; RB = Riparo del Broion; SB = Grotta di San Bernardino; RS = Grotta del Rio Secco.

\begin{tabular}{|c|c|c|c|c|c|c|c|c|c|c|c|c|c|c|c|c|c|c|c|c|}
\hline \multirow[t]{2}{*}{ Taxa } & \multicolumn{2}{|c|}{$\begin{array}{l}\text { RS } 5+8 \text { - } \\
\mathrm{LM}\end{array}$} & \multicolumn{2}{|c|}{ RF A9 - LM } & \multicolumn{2}{|c|}{ RF A6 - LM } & \multicolumn{2}{|c|}{$\begin{array}{l}\mathrm{RF} A 5 / \mathrm{A} 5+\mathrm{A} 6- \\
\mathrm{LM}\end{array}$} & \multicolumn{2}{|c|}{$\begin{array}{l}\text { RS 5top + } 7 \text { - } \\
\text { LM }\end{array}$} & \multicolumn{2}{|c|}{$\begin{array}{l}\text { SB II + III - } \\
\text { LM }\end{array}$} & \multicolumn{2}{|c|}{ RF A4 - LM } & \multicolumn{2}{|c|}{$\begin{array}{l}\text { RB } 1 e+1 f+1 g- \\
\text { UL }\end{array}$} & \multicolumn{2}{|c|}{ RF A3 - UL } & \multicolumn{2}{|c|}{ RF A2-A2R - PA } \\
\hline & Nisp & $\%$ & Nisp & $\%$ & Nisp & $\%$ & Nisp & $\%$ & Nisp & $\%$ & Nisp & $\%$ & Nisp & $\%$ & Nisp & $\%$ & Nisp & $\%$ & Nisp & $\%$ \\
\hline Canis lupus & 3 & 1.3 & 4 & 11.1 & 7 & 11.9 & 4 & 7.7 & & & 3 & 1.8 & 11 & 11.5 & & & 21 & 25.6 & 38 & 37.3 \\
\hline Vulpes vulpes & 3 & 1.3 & 6 & 16.7 & 20 & 33.9 & 26 & 50 & 1 & 0.5 & 9 & 5.5 & 61 & 63.5 & 4 & 9.5 & 36 & 43.9 & 28 & 27.5 \\
\hline Vulpes/alopex & & & & & & & 3 & 5.8 & & & & & 5 & 5.2 & & & 6 & 7.3 & 7 & 6.9 \\
\hline Alopex lagopus & & & & & & & & & & & & & & & & & & & 2 & 2 \\
\hline Ursus spelaeus & 157 & 66.2 & 8 & 22.2 & & & 2 & 3.8 & 148 & 66.7 & 100 & 61.3 & 2 & 2.1 & 21 & 50 & & & 1 & 1 \\
\hline Ursus arctos & 1 & 0.4 & 4 & 11.1 & 10 & 16.9 & 4 & 7.7 & 6 & 2.7 & 3 & 1.8 & 11 & 11.5 & & & 7 & 8.5 & 1 & 1 \\
\hline Ursus sp. & 64 & 27 & 5 & 13.9 & 21 & 35.6 & 13 & 25 & 66 & 29.7 & 38 & 23.3 & 2 & 2.1 & 13 & 31 & 2 & 2.4 & 1 & 1 \\
\hline Mustela erminea & 4 & 1.7 & & & & & & & & & & & & & & & 1 & 1.2 & 2 & 2 \\
\hline Mustela nivalis & & & 4 & 11.1 & 1 & 1.7 & & & & & & & 1 & 1 & & & 2 & 2.4 & 1 & 1 \\
\hline Mustela putorius & & & & & & & & & & & 2 & 1.2 & & & & & & & 1 & 1 \\
\hline Martes martes & 3 & 1.3 & & & & & & & 1 & 0.5 & & & & & 1 & 2.4 & & & & \\
\hline Mustelidae & & & 1 & 2. & & & & & & & & & & & & & & & & \\
\hline Gulo gulo & & & & & & & & & & & & & 1 & 1 & & & 3 & 3.7 & 1 & 1 \\
\hline Meles meles & 2 & 0.8 & & & & & & & & & & & & & & & & & & \\
\hline Crocuta crocuta spelaea & & & 3 & 8.3 & & & & & & & & & & & & & 3 & 3.7 & 15 & 14.7 \\
\hline Felis silvestris & & & & & & & & & & & 2 & 1.2 & & & 2 & 4.8 & & & & \\
\hline Lynx lynx & & & & & & & & & & & 4 & 2.5 & & & & & 1 & 1.2 & 3 & 2.9 \\
\hline Panthera pardus & & & & & & & & & & & 1 & 0.6 & 2 & 2.1 & & & & & & \\
\hline Panthera leo spelaea & & & 1 & 2.8 & & & & & & & & & & & & & & & 1 & 1 \\
\hline Felidae & & & & & & & & & & & 1 & 0.6 & & & 1 & 2.4 & & & & \\
\hline Total Carnivora & 237 & & 36 & & 59 & & 52 & & 222 & & 163 & & 96 & & 42 & & 82 & & 102 & \\
\hline
\end{tabular}

Table A.3

Total Nisp and relative frequency of Rodentia and Lagomorpha documented in levels and layers of Northern Italy (Area 1 in Fig.1) presented in chronological-cultural order. $\mathrm{RF}=$ Grotta di Fumane; $\mathrm{RB}=$ Riparo del Broion; SB = Grotta di San Bernardino; RS = Grotta del Rio Secco.

\begin{tabular}{|c|c|c|c|c|c|c|c|c|c|c|}
\hline Taxa & $\begin{array}{l}\text { RS } 5+8- \\
\text { LM }\end{array}$ & $\begin{array}{l}\text { RF A9 - } \\
\text { LM }\end{array}$ & $\begin{array}{l}\text { RF A6 - } \\
\text { LM }\end{array}$ & $\begin{array}{l}\text { RF A5/A5+A6 - } \\
\mathrm{LM}\end{array}$ & $\begin{array}{l}\text { RS 5top + } 7- \\
\text { LM }\end{array}$ & $\begin{array}{l}\text { SB II + III - } \\
\mathrm{LM}\end{array}$ & $\begin{array}{l}\text { RF A4 - } \\
\text { LM }\end{array}$ & $\begin{array}{l}\text { RB } 1 e+1 f+1 g- \\
\text { UL }\end{array}$ & $\begin{array}{l}\text { RF A3 - } \\
\text { UL }\end{array}$ & RF A2-A2R - PA \\
\hline & Nisp & Nisp & Nisp & Nisp & Nisp & Nisp & Nisp & Nisp & Nisp & Nisp \\
\hline Marmota marmota & & 8 & 1 & & & 18 & & 3 & 2 & 2 \\
\hline Lepus cfr. timidus & & & & & & & & 2 & & 4 \\
\hline Lepus sp. & 1 & & & 1 & & 3 & & & 3 & 1 \\
\hline Castor fiber & & & & & & 27 & & 1 & & 1 \\
\hline $\begin{array}{l}\text { Total Lagomorpha and Ro- } \\
\text { dentia }\end{array}$ & 1 & 8 & 1 & 1 & 0 & 48 & 0 & 6 & 5 & 8 \\
\hline
\end{tabular}




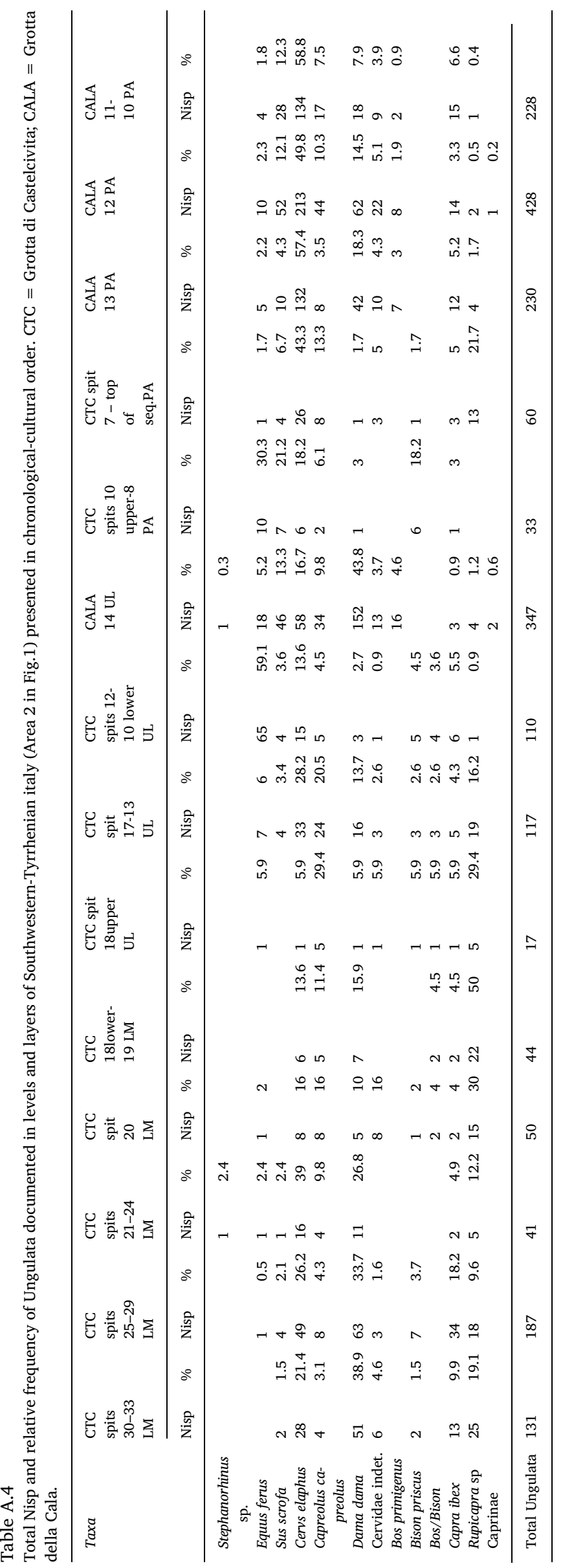


Table A.5

Total Nisp and relative frequency of Carnivora documented in levels and layers of Southwestern-Tyrrhenian italy (Area 2 in Fig.1) presented in chronological-cultural order. CTC = Grotta di Castelcivita; CALA = Grotta della Cala.

\begin{tabular}{|c|c|c|c|c|c|c|c|c|c|c|c|}
\hline Taxa & $\begin{array}{l}\text { CTC gar } \\
\text { LM }\end{array}$ & $\begin{array}{l}\text { CTC lower rsi } \\
\text { LM }\end{array}$ & $\begin{array}{l}\text { CTC spit } 18 \text { upper } \\
\text { UL }\end{array}$ & $\begin{array}{l}\text { CTC spits } 17-13 \\
\text { UL }\end{array}$ & $\begin{array}{l}\text { CTC spits } 12-10 \\
\text { lower UL }\end{array}$ & $\begin{array}{l}\text { CALA } 14 \\
\text { UL }\end{array}$ & $\begin{array}{l}\text { CTC } \\
\text { PA }\end{array}$ & $\begin{array}{l}\text { CALA } \\
13 \text { PA }\end{array}$ & $\begin{array}{l}\text { CALA } \\
12 \text { PA }\end{array}$ & $\begin{array}{l}\text { CALA } \\
11 \text { PA }\end{array}$ & $\begin{array}{l}\text { CALA } \\
10 \mathrm{PA}\end{array}$ \\
\hline & Nisp & Nisp & Nisp & Nisp & Nisp & Nisp & Nisp & Nisp & Nisp & Nisp & Nisp \\
\hline Canis lupus & & & & & 1 & & 2 & 2 & 1 & & \\
\hline Vulpes vulpes & & & & 2 & 2 & 9 & & 2 & 1 & & \\
\hline Ursus spelaeus & & 1 & & & 1 & & & & & & \\
\hline Ursus arctos & & & & 4 & & 7 & & & 2 & 1 & \\
\hline Mustela nivalis & 1 & & & 1 & 2 & & & & & & \\
\hline Martes sp. & & & & & & 14 & & & 1 & & \\
\hline Mustelidae & & & & 2 & & & & & & & \\
\hline Meles meles & & & & 2 & & & & & & & \\
\hline $\begin{array}{l}\text { Crocuta crocuta s- } \\
\text { pelaea }\end{array}$ & 11 & & & 1 & 6 & & 1 & & & & \\
\hline Felis silvestris & & & & 2 & 3 & 5 & & 2 & & & \\
\hline $\begin{array}{l}\text { Panthera pardus } \\
\text { Panthera leo spel. }\end{array}$ & 3 & 1 & & 3 & 2 & 17 & & 4 & 6 & 3 & \\
\hline Carnivora indet. & 6 & 5 & 1 & 7 & 1 & 3 & 2 & 1 & 1 & 1 & \\
\hline Total Carnivora & 21 & 7 & 1 & 24 & 18 & 55 & 5 & 11 & 12 & 5 & 0 \\
\hline
\end{tabular}

Table A.6

Total Nisp and relative frequency of Ungulata documented in levels and layers of Southeastern (Ionian-Adriatic) Italy (Area 3 in Fig.1) presented in chronologicalcultural order. $\mathrm{CAV}=$ Grotta del Cavallo; OSC $=$ Riparo l'Oscurusciuto.

\begin{tabular}{|c|c|c|c|c|c|c|c|c|c|c|c|c|c|c|c|c|}
\hline \multirow[t]{2}{*}{$>$ Taxa } & \multicolumn{2}{|c|}{$\begin{array}{l}\text { CAV FIIIeb-e } \\
\text { LM }\end{array}$} & \multicolumn{2}{|l|}{$\begin{array}{l}\text { CAV FIIIb-c- } \\
\text { d LM }\end{array}$} & \multicolumn{2}{|l|}{$\begin{array}{l}\text { CAV FI-II- } \\
\text { IIIa LM }\end{array}$} & \multicolumn{2}{|l|}{$\begin{array}{l}\text { OSC 4-13 } \\
\text { LM }\end{array}$} & \multicolumn{2}{|l|}{$\begin{array}{l}\text { OSC } 3 \\
\text { LM }\end{array}$} & \multicolumn{2}{|l|}{$\begin{array}{l}\text { OSC 2-29-30- } \\
31 \text { LM }\end{array}$} & \multicolumn{2}{|l|}{$\begin{array}{l}\text { OSC } 1 \\
\text { LM }\end{array}$} & \multicolumn{2}{|c|}{$\begin{array}{l}\text { CAV EIII } 5 \\
\text { UL }\end{array}$} \\
\hline & Nisp & $\%$ & Nisp & $\%$ & Nisp & $\%$ & Nisp & $\%$ & Nisp & $\%$ & Nisp & $\%$ & Nisp & $\%$ & Nisp & $\%$ \\
\hline $\begin{array}{l}\text { Stephanorhinus s- } \\
\text { p. }\end{array}$ & & & & & & & 1 & 0.2 & 7 & 12.3 & & & & & & \\
\hline Equus ferus & 40 & 11.5 & 40 & 14.9 & 50 & 19.8 & 17 & 3 & 16 & 28.1 & 48 & 25.9 & 2 & 5 & 53 & 27.3 \\
\hline $\begin{array}{l}\text { Equus hydrun- } \\
\text { tinus }\end{array}$ & & & & & & & & & & & & & & & 1 & 0.5 \\
\hline Equus sp. & & & & & & & & & & & & & & & 1 & 0.5 \\
\hline Sus scrofa & 1 & 0.3 & 4 & 1.5 & 2 & 0.8 & 1 & 0.2 & & & & & & & 1 & 0.5 \\
\hline Cervus elaphus & 72 & 20.6 & 54 & 20.1 & 69 & 27.3 & 51 & 8.9 & 15 & 26.3 & 27 & 14.6 & 11 & 27.5 & 52 & 26.8 \\
\hline $\begin{array}{c}\text { Capreolus ca- } \\
\text { preolus }\end{array}$ & & & 6 & 2.2 & 3 & 1.2 & 11 & 1.9 & 2 & 3.5 & 8 & 4.3 & 1 & 2.5 & & \\
\hline Dama dama & 7 & 2 & 83 & 31 & 20 & 7.9 & 38 & 6.6 & 1 & 1.8 & 12 & 6.5 & 6 & 15 & & \\
\hline Cervidae indet. & 2 & 0.6 & 15 & 5.6 & 8 & 3.2 & 6 & 1 & & & 3 & 1.6 & & & & \\
\hline Bos primigenus & 227 & 65 & 66 & 24.6 & 101 & 39.9 & 445 & 77.5 & 15 & 26.3 & 82 & 44.3 & 20 & 50 & 86 & 44.3 \\
\hline Capra ibex & & & & & & & 1 & 0.2 & 1 & 1.8 & 5 & 2.7 & & & & \\
\hline Rupicapra sp. & & & & & & & 3 & 0.5 & & & & & & & & \\
\hline Total Nisp & 349 & & 268 & & 253 & & 574 & & 57 & & 185 & & 40 & & 194 & \\
\hline
\end{tabular}

Table A.7

Total Nisp and relative frequency of Carnivora documented in levels and layers of Southeastern (Ionian-Adriatic) Italy (Area 3 in Fig.1) presented in chronologicalcultural order. CAV $=$ Grotta del Cavallo; OSC $=$ Riparo l'Oscurusciuto.

\begin{tabular}{|c|c|c|c|c|c|c|c|}
\hline \multirow[t]{2}{*}{ Taxa } & CAV FIII LM & CAV F II LM & OSC US 4-13 LM & OSC US 3 LM & OSC US 2-29-31 LM & OSC US $1 \mathrm{LM}$ & CAV EIII5 UL \\
\hline & Nisp & Nisp & Nisp & Nisp & Nisp & Nisp & Nisp \\
\hline Canis lupus & & & 1 & & & & 2 \\
\hline Vulpes vulpes & 42 & 13 & & & & & 4 \\
\hline Ursus spelaeus & & & & & & & \\
\hline Ursus arctos & & & & & & & \\
\hline Mustela nivalis & & & & & & & \\
\hline Martes sp. & & & & & & & \\
\hline Mustelidae & & & & & & & \\
\hline Meles meles & & & & & & & \\
\hline Crocuta crocuta spelaea & & & & & & & 1 \\
\hline Felis silvestris & 2 & & & & & & \\
\hline Panthera pardus & & & & & & & \\
\hline Panthera leo spelaea & & & & & 1 & & \\
\hline Carnivora indet. & 2 & & & & & & \\
\hline Total Carnivora & 46 & 13 & 1 & 0 & 1 & 0 & 7 \\
\hline
\end{tabular}




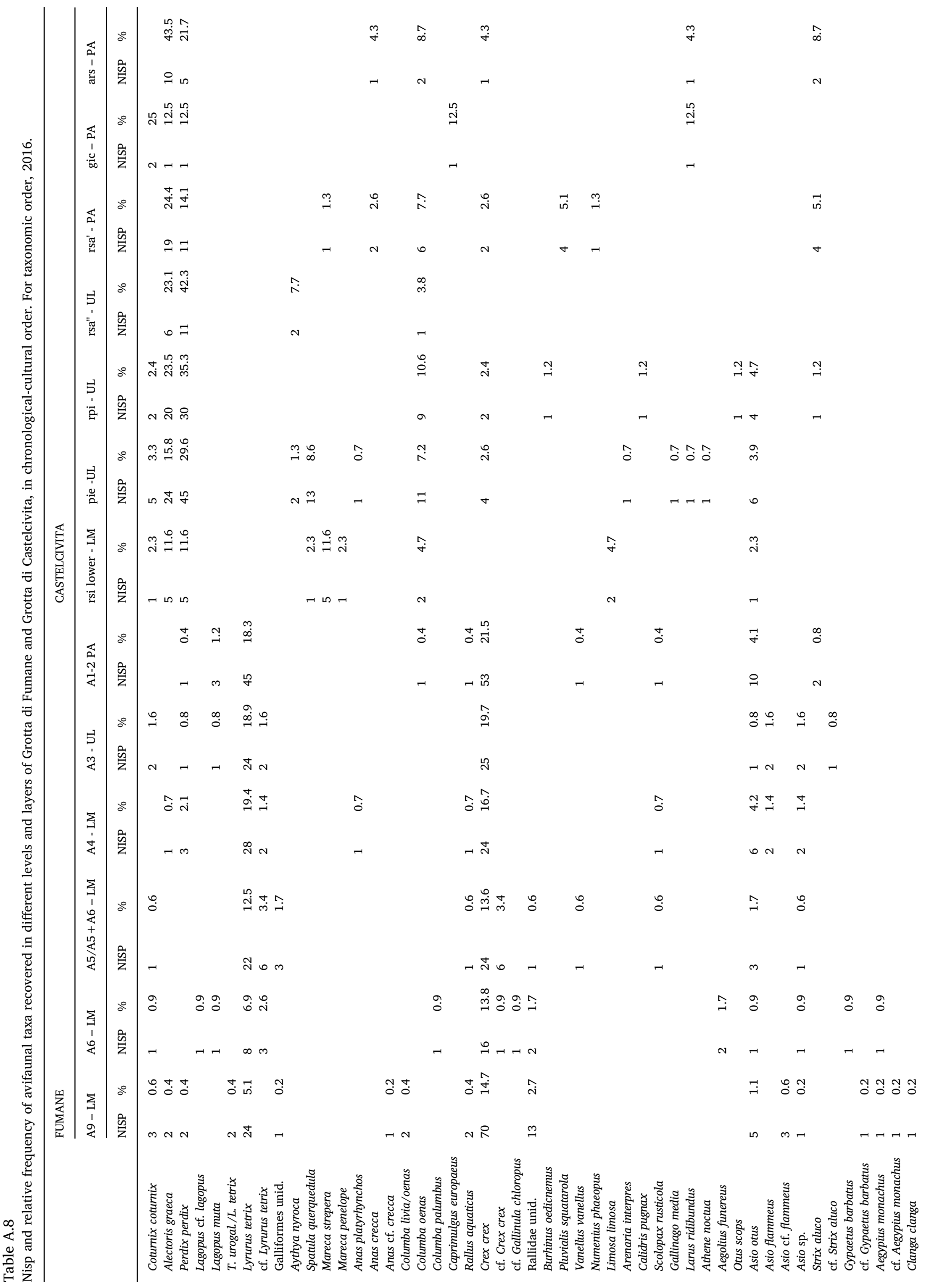




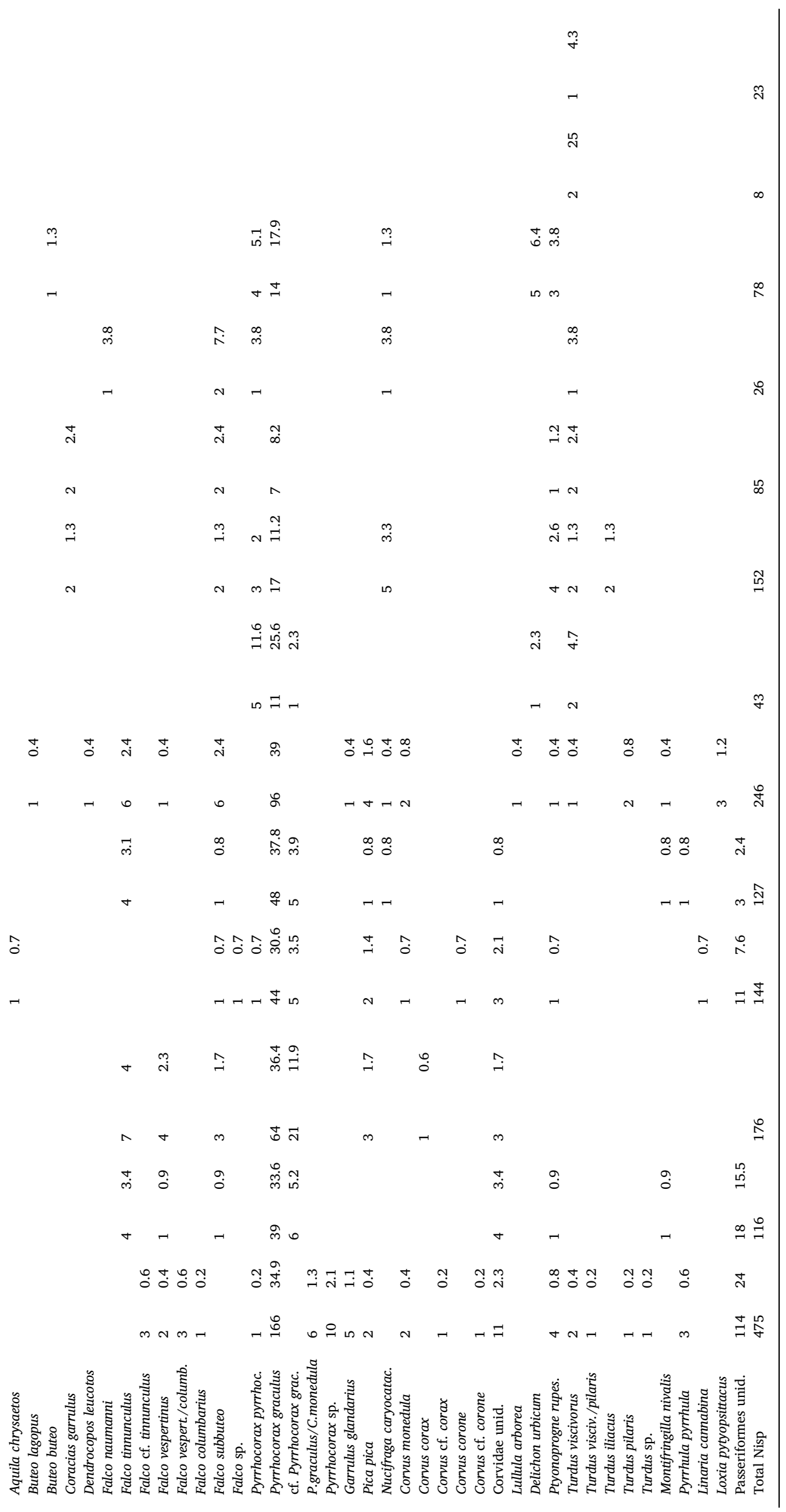


Table A.9

The most recently available dates for the context of interest used in the present work.

\begin{tabular}{|c|c|c|c|c|c|c|c|c|c|c|}
\hline & site & Code(s) & Material & Method & Level & Detailed level & Technocomplex & 14CAge & sd & Reference \\
\hline 1 & Fumane & OxA-11347 & Charcoal & ABA14C & $\mathrm{A} 2$ & A2; sq. 97d & Protoaurignacian & 30650 & 260 & Higham et al., (2009); Higham (2011) \\
\hline 2 & Fumane & OxA-17569 & Charcoal & $\begin{array}{l}\text { ABOx- } \\
\text { SC_14C }\end{array}$ & $\mathrm{A} 2$ & A2; sq. 97d & Protoaurignacian & 35640 & 220 & Higham et al., (2009); Higham (2011) \\
\hline 3 & Fumane & OxA-11360 & Charcoal & ABA14C & $\mathrm{A} 2$ & A2; sq. 107i & Protoaurignacian & 31830 & 260 & Higham et al., (2009); Higham (2011) \\
\hline 4 & Fumane & OxA-17570 & Charcoal & $\begin{array}{l}\text { ABOx- } \\
\text { SC_14C }\end{array}$ & $\mathrm{A} 2$ & A2; sq. $107 i$ & Protoaurignacian & 35180 & 220 & Higham et al., (2009); Higham (2011) \\
\hline 5 & Fumane & OxA-19411 & Charcoal & ABA14C & A2 & A2/struc. 17 & Protoaurignacian & 32530 & 240 & Higham et al., (2009); Higham (2011) \\
\hline 6 & Fumane & OxA-19413 & Charcoal & ABA14C & A2 & $\begin{array}{l}\text { A2/struc.16/ } \\
\text { lev.B }\end{array}$ & Protoaurignacian & 32120 & 240 & Higham et al., (2009); Higham (2011) \\
\hline 7 & Fumane & OxA-19414 & Charcoal & $\begin{array}{l}\text { ABOx- } \\
\text { SC_14C }\end{array}$ & $\mathrm{A} 2$ & $\begin{array}{l}\text { A2/struc.16/ } \\
\text { lev.Ba }\end{array}$ & Protoaurignacian & 34180 & 270 & Higham et al., (2009); Higham (2011) \\
\hline 8 & Fumane & OxA-19412 & Charcoal & $\begin{array}{l}\text { ABOx- } \\
\text { SC_14C }\end{array}$ & $\mathrm{A} 2$ & A2/struc.17a & Protoaurignacian & 34940 & 280 & Higham et al., (2009); Higham (2011) \\
\hline 9 & Fumane & OxA-19525 & Charcoal & ABA14C & A2 & A2/struc. 18 & Protoaurignacian & 33380 & 210 & Higham et al., (2009); Higham (2011) \\
\hline 10 & Fumane & OxA-19584 & Charcoal & $\begin{array}{l}\text { ABOx- } \\
\text { SC_14C }\end{array}$ & $\mathrm{A} 2$ & A2/struc.18 & Protoaurignacian & 35850 & 310 & Higham et al., (2009); Higham (2011) \\
\hline 11 & Fumane & Oxa-21736 & $\begin{array}{l}\text { Mammal } \\
\text { Bone }\end{array}$ & $\begin{array}{l}\text { 14C-ultrafil- } \\
\text { tration }\end{array}$ & A3 & A3 & Uluzzian & 39100 & 1000 & Douka et al. (2014) \\
\hline 12 & Fumane & Oxa-X-2295-52 & $\begin{array}{l}\text { Mammal } \\
\text { Bone }\end{array}$ & $\begin{array}{l}\text { 14C-ultrafil- } \\
\text { tration }\end{array}$ & A3 & A3 & Uluzzian & 41300 & 1300 & Douka et al. (2014) \\
\hline 13 & Fumane & Oxa-21735 & $\begin{array}{l}\text { Mammal } \\
\text { Bone }\end{array}$ & $\begin{array}{l}\text { 14C-ultrafil- } \\
\text { tration }\end{array}$ & A4 & $\begin{array}{l}\text { A4/struct. II/ } \\
744\end{array}$ & Late Mousterian & 42000 & 1700 & Douka et al. (2014) \\
\hline 14 & Fumane & Oxa-21733 & $\begin{array}{l}\text { Mammal } \\
\text { Bone }\end{array}$ & $\begin{array}{l}\text { 14C-ultrafil- } \\
\text { tration }\end{array}$ & A4 & A4II & Late Mousterian & 41000 & 1300 & Douka et al. (2014) \\
\hline 15 & Fumane & Oxa-21734 & $\begin{array}{l}\text { Mammal } \\
\text { Bone }\end{array}$ & $\begin{array}{l}\text { 14C-ultrafil- } \\
\text { tration }\end{array}$ & A4 & A4II & Late Mousterian & 42000 & 1400 & Douka et al. (2014) \\
\hline 16 & Fumane & OxA-17566 & Charcoal & $\begin{array}{l}\text { ABOx- } \\
\text { SC_14C }\end{array}$ & A5 & A5 + A6, sq.90 & Late Mousterian & 40460 & 360 & Higham et al., (2009); Higham (2011) \\
\hline 17 & Fumane & OxA-17567 & Charcoal & ABA14C & A5 & A5 + A6, sq.90 & Late Mousterian & 39500 & 330 & Higham et al., (2009); Higham (2011) \\
\hline 18 & Fumane & OxA-17568 & Charcoal & ABA14C & A5 & A5 + A6, sq.90 & Late Mousterian & 39490 & 350 & Higham et al., (2009); Higham (2011) \\
\hline 19 & Fumane & OxA-8022 & Charcoal & $\mathrm{ABA} 14 \mathrm{C}$ & A5 & A5 + A6, sq.90 & Late Mousterian & 38800 & 750 & Higham et al., (2009); Higham (2011) \\
\hline 20 & Fumane & OxA-8023 & Charcoal & ABA14C & A5 & A5 + A6, sq.90 & Late Mousterian & 38250 & 700 & Higham et al., (2009); Higham (2011) \\
\hline 21 & Fumane & OxA-19410 & Charcoal & ABA14C & A5 & $\begin{array}{l}\text { A5 sq. } \\
88 i, 3789 / \\
\text { struc.III }\end{array}$ & Late Mousterian & 34500 & 270 & Higham et al., (2009); Higham (2011) \\
\hline 22 & Fumane & OxA-X-2275-45 & Charcoal & $\begin{array}{l}\text { ABOx- } \\
\text { SC_14C }\end{array}$ & A5 & $\begin{array}{l}\text { A5 sq. } \\
88 i, 3789 / \\
\text { struc.III }\end{array}$ & Late Mousterian & 41650 & 650 & Higham et al., (2009); Higham (2011) \\
\hline 23 & Fumane & OxA-17980 & Charcoal & $\begin{array}{l}\text { ABOx- } \\
\text { SC_14C }\end{array}$ & A5 & $\begin{array}{l}\text { A5, sqs. } \\
85,86,95,96\end{array}$ & Late Mousterian & 40150 & 350 & Higham et al., (2009); Higham (2011) \\
\hline 24 & Fumane & OxA-18199 & Charcoal & ABA14C & A5 & $\begin{array}{l}\text { A5, sqs. } \\
85,86,95,96\end{array}$ & Late Mousterian & 36860 & 700 & Higham et al., (2009); Higham (2011) \\
\hline 25 & Fumane & OxA-6463 & Charcoal & ABA14C & A5 & $\begin{array}{l}\text { A5, sqs. } \\
85,86,95,96\end{array}$ & Late Mousterian & 33700 & 600 & Higham et al., (2009); Higham (2011) \\
\hline 26 & Fumane & OxA-21796 & Bone & $\begin{array}{l}\text { 14C-ultrafil- } \\
\text { tration }\end{array}$ & $\mathrm{A} 2$ & & Protoaurignacian & 35400 & 750 & Higham (2011) \\
\hline 27 & Fumane & OxA-21712 & Bone & $\begin{array}{l}\text { 14C-ultrafil- } \\
\text { tration }\end{array}$ & A5 & & Late Mousterian & 40000 & 1100 & Higham (2011) \\
\hline 28 & Fumane & OxA-21809 & Bone & $\begin{array}{l}\text { 14C-ultrafil- } \\
\text { tration }\end{array}$ & A5 & $\mathrm{A} 5+\mathrm{A} 6$ & Late Mousterian & 40200 & 1200 & Higham (2011) \\
\hline 29 & Fumane & OxA-21758 & Bone & $\begin{array}{l}\text { 14C-ultrafil- } \\
\text { tration }\end{array}$ & A5 & $\mathrm{A} 5+\mathrm{A} 6$ & Late Mousterian & 41100 & 1300 & Higham (2011) \\
\hline 30 & Fumane & OxA-21757 & Bone & $\begin{array}{l}\text { 14C-ultrafil- } \\
\text { tration }\end{array}$ & A5 & $\mathrm{A} 5+\mathrm{A} 6$ & Late Mousterian & 41500 & 1500 & Higham (2011) \\
\hline 31 & R. Broion & OxA-35527 & Bone & $14 \mathrm{C}$ & $1 g$ & $1 g$ & Uluzzian & 38900 & 1000 & Peresani et al. (2019) \\
\hline 32 & Rio Secco & $\begin{array}{l}\text { S-EVA25353/ } \\
\text { MAMS15230 }\end{array}$ & $\begin{array}{l}\text { Bone with } \\
\text { cutmarks }\end{array}$ & $14 \mathrm{C}$ & 5 top & I14 b & Mousterian & 44100 & 660 & Talamo et al. (2014) \\
\hline 33 & Rio Secco & $\begin{array}{l}\text { S-EVA25355/ } \\
\text { MAMS15231 }\end{array}$ & $\begin{array}{l}\text { Bone with } \\
\text { cutmarks }\end{array}$ & $14 \mathrm{C}$ & 5 top I & G14III & Mousterian & 45695 & 790 & Talamo et al. (2014) \\
\hline 34 & Rio Secco & $\begin{array}{l}\text { S-EVA25356/ } \\
\text { MAMS15232 }\end{array}$ & Bone & $14 \mathrm{C}$ & 5 top II & H14IV & Mousterian & 43210 & 600 & Talamo et al. (2014) \\
\hline 35 & Rio Secco & $\begin{array}{l}\text { S-EVA25357/ } \\
\text { MAMS15233 }\end{array}$ & $\begin{array}{l}\text { Bone with } \\
\text { cutmarks }\end{array}$ & $14 \mathrm{C}$ & 5 top I & I14II & Mousterian & 45740 & 800 & Talamo et al. (2014) \\
\hline 36 & Rio Secco & $\begin{array}{l}\text { S-EVA25359/ } \\
\text { MAMS15235 }\end{array}$ & Bone & $14 \mathrm{C}$ & 7 & $\mathrm{H} 14 \mathrm{~h}$ & Mousterian & 46320 & 1430 & Talamo et al. (2014) \\
\hline 37 & Rio Secco & $\begin{array}{l}\text { S-EVA25361/ } \\
\text { MAMS15236 }\end{array}$ & $\begin{array}{l}\text { Bone with } \\
\text { cutmarks }\end{array}$ & $14 \mathrm{C}$ & 7 & H13IV & Mousterian & $>49000$ & & Talamo et al. (2014) \\
\hline 38 & Rio Secco & $\begin{array}{l}\text { S-EVA25362/ } \\
\text { MAMS15237 }\end{array}$ & $\begin{array}{l}\text { Bone with } \\
\text { cutmarks }\end{array}$ & $14 \mathrm{C}$ & 7 & H13IV & Mousterian & 44560 & 1150 & Talamo et al. (2014) \\
\hline 39 & Rio Secco & $\begin{array}{l}\text { S-EVA25363/ } \\
\text { MAMS15238 }\end{array}$ & $\begin{array}{l}\text { Bone with } \\
\text { cutmarks }\end{array}$ & $14 \mathrm{C}$ & 7 & $\mathrm{H} 14 \mathrm{~g}$ & Mousterian & 44770 & 1180 & Talamo et al. (2014) \\
\hline 40 & Rio Secco & OxA-25359 & Charcoal & $14 \mathrm{C}$ & 8 & sq.H11IV n.17 & Mousterian & 42000 & 900 & $\begin{array}{l}\text { Peresani et al. (2014); Talamo et al. } \\
\text { (2014) }\end{array}$ \\
\hline 41 & Rio Secco & LTL429A & Bone & $14 \mathrm{C}$ & 5 top II & GRSI & Mousterian & 37790 & 360 & $\begin{array}{l}\text { Peresani et al. (2014); Talamo et al. } \\
\text { (2014) }\end{array}$ \\
\hline
\end{tabular}




\begin{tabular}{|c|c|c|c|c|c|c|c|c|c|c|}
\hline 42 & S.Bernardino & & & U/Th ESR & II & & Mousterian & 52000 & 5000 & \\
\hline 43 & S.Bernardino & & & U/Th ESR & II & & Mousterian & 38000 & 5000 & $\begin{array}{l}\text { Gruppioni (2003); López-García (2017) } \\
\text { Peresani et al. (2015) }\end{array}$ \\
\hline 44 & S.Bernardino & & & U/Th ESR & II & & Mousterian & 35000 & 4000 & $\begin{array}{l}\text { Gruppioni (2003); López-García et al. } \\
\text { (2017) }\end{array}$ \\
\hline 45 & S.Bernardino & & & U/Th ESR & II & & Mousterian & 49000 & 5000 & \\
\hline 46 & S.Bernardino & & & U/Th ESR & II & & Mousterian & 54000 & 5000 & \\
\hline 47 & Castelcivita & GrN-13984 & Charcoal & $14 \mathrm{C}$ & $\operatorname{cgr}$ & spits $29-30$ & Late Mousterian & 42700 & 900 & Gambassini (1997) \\
\hline 48 & Castelcivita & GrN-13982 & Charcoal & $14 \mathrm{C}$ & $\operatorname{cgr}$ & spits $29-30$ & Late Mousterian & 39100 & 1300 & Gambassini (1997) \\
\hline 49 & Castelcivita & Oxa-22622 & Charcoal & $\begin{array}{l}\text { ABOx- } \\
\text { SC_14C }\end{array}$ & rsa" & spit 11 & Uluzzian & 36120 & 360 & Wood et al. (2012) \\
\hline 50 & Oscurusciuto & Beta 181165 & $\begin{array}{l}\text { Mammal } \\
\text { bone }\end{array}$ & $14 \mathrm{C}$ & 1 & 1 & Late Mousterian & 38500 & 900 & Marciani et al. (2016) \\
\hline 51 & Oscurusciuto & & Tephra & $\mathrm{Ar}^{40} / \mathrm{Ar}^{39}$ & 14 & 1-TM19 & Mousterian & $\sim 55000$ & & Spagnolo et al. (2016) \\
\hline 57 & Cavallo & Oxa-19254 & Shell & $14 \mathrm{C}$ & $\mathrm{D} 1=\mathrm{DIb}$ & & Uluzzian & 35080 & 230 & Benazzi et al. (2011) \\
\hline 58 & Cavallo & Oxa-19255 & Shell & $14 \mathrm{C}$ & $\mathrm{D} 2=\mathrm{DIb}$ & & Uluzzian & 36260 & 250 & Benazzi et al. (2011) \\
\hline 59 & Cavallo & Oxa-20631 & Shell & $14 \mathrm{C}$ & DII & & Uluzzian & 36780 & 310 & Benazzi et al. (2011) \\
\hline 60 & Cavallo & Oxa-19257 & Shell & $14 \mathrm{C}$ & D3 = DII & & Uluzzian & 42360 & 400 & Benazzi et al. (2011) \\
\hline 61 & Cavallo & Oxa-19258 & Shell & $14 \mathrm{C}$ & D8 = DII? & & Uluzzian & 36000 & 400 & Benazzi et al. (2011) \\
\hline 62 & Cavallo & Oxa-19256 & Shell & $14 \mathrm{C}$ & $\mathrm{E} 1=\mathrm{E}-\mathrm{D}$ & & Uluzzian & 39060 & 310 & Benazzi et al. (2011) \\
\hline 63 & Cavallo & Oxa-X2280-16 & Shell & $14 \mathrm{C}$ & $\mathrm{E} 1=\mathrm{E}-\mathrm{D}$ & & Uluzzian & 38300 & 400 & Benazzi et al. (2011) \\
\hline 64 & Cavallo & Oxa-19242 & Shell & $14 \mathrm{C}$ & $\mathrm{E} 4=$ EII-I & & Uluzzian & 39990 & 340 & Benazzi et al. (2011) \\
\hline 65 & Cavallo & Fi0822 & Charcoal & ABA14C & FII & & Mousterian & 42000 & 2400 & Fabbri et al. (2016) \\
\hline 66 & Cavallo & Fi0824 & Charcoal & ABA14C & FII & & Mousterian & 39300 & 1900 & Fabbri et al. (2016) \\
\hline 67 & Cavallo & & Tephra & $\mathrm{Ar}^{40} / \mathrm{Ar}^{39}$ & Fa-Y6 & & & 45500 & 1000 & Zanchetta et al. (2018) \\
\hline 68 & Cavallo & & Tephra & $\mathrm{Ar}^{40} / \mathrm{Ar}^{39}$ & CII-Y5 & & & 39850 & 140 & Zanchetta et al. (2018) \\
\hline
\end{tabular}

Most recent available absolute datings for the sampled archaeological sites.

Table A.10

Number and \% of single teeth and small limb bones of Ungulata uncovered in different levels of the Late Mousterian, Uluzzian and Protoaurignacian sites of Southern Italy. CALA $=$ Grotta della Cala; CTC $=$ Grotta di Castelcivita; CAV $=$ Grotta del Cavallo; OSC $=$ Riparo l'Oscurusciuto.

\begin{tabular}{|c|c|c|c|c|c|c|c|}
\hline \multirow[t]{2}{*}{ Sites levels - US } & \multicolumn{2}{|c|}{ Single teeth } & \multicolumn{2}{|c|}{ Carpal and tarsal bones } & \multicolumn{2}{|c|}{ Phalanges + sesamoides } & \multirow{2}{*}{$\begin{array}{l}\text { Total ungulates } \\
\text { Nisp }\end{array}$} \\
\hline & NR & $\%$ & NR & $\%$ & NR & $\%$ & \\
\hline CALA PA & 299 & 35.4 & 188 & 22.3 & 115 & 13.6 & 844 \\
\hline CTC PA & 2 & 5.6 & 4 & 11.1 & 5 & 13.9 & 38 \\
\hline CALA UL & 137 & 41.5 & 31 & 9.4 & 41 & 12.4 & 331 \\
\hline CAV EIII5 UL & 46 & 23.5 & 30 & 15.3 & 59 & 30.1 & 196 \\
\hline CTC UL & 38 & 15.1 & 13 & 5.2 & 38 & 15.1 & 233 \\
\hline OSC US 2 LM & 128 & 69.2 & 3 & 1.6 & 5 & 2.7 & 185 \\
\hline CAV str. F LM & 552 & 65.3 & 12 & 1.4 & 67 & 7.9 & 845 \\
\hline CTC LM & 6 & 9 & 3 & 4.5 & 9 & 13.4 & 67 \\
\hline
\end{tabular}

Table A.11

Percentages of determinated skeletal parts in the taxonomically indeterminate remains recovered from the different Late Mousterian and Uluzzian layers and levels sampled in Southern Italy. OSC = Riparo l'Oscurusciuto; CAV = Grotta del Cavallo; CTC = Grotta di Castelcivita.

\begin{tabular}{|c|c|c|c|c|}
\hline \multirow[t]{2}{*}{ Elements } & OSC US 4/1 LM & CAV FII LM & CTC LM & CAV EIII5 UL \\
\hline & $\%$ & $\%$ & $\%$ & $\%$ \\
\hline Antler/Horn & 0.4 & 0.3 & & 3.5 \\
\hline Skull & 4.3 & 4.8 & 4.9 & 2.5 \\
\hline Mandible & 0.4 & 0.4 & 2 & 0.7 \\
\hline Teeth & 18.4 & 14.9 & 3.6 & 7.9 \\
\hline Vertebrae & & 3 & 4.6 & 3.3 \\
\hline Ribs & 3.5 & 11.2 & 15.7 & 13.4 \\
\hline Scapula & & 2.3 & & 0.4 \\
\hline Sternum & & & 0.7 & 0.7 \\
\hline Pelvis & 0.4 & & 0.3 & 0.2 \\
\hline Metapodials & & & 0.8 & \\
\hline Diaphysis & 45.3 & 41.3 & 41.4 & 18.6 \\
\hline Epiphysis & 5.6 & 8.2 & 8,2 & 12,9 \\
\hline Spongy bones & 21.8 & 11.6 & 12 & 31.9 \\
\hline Total remains & 5747 & 9574 & 1920 & 5185 \\
\hline
\end{tabular}


Table A.12

Detail and percentages of remains that present with evidence of digestion and/or gnawing by carnivores documented in the levels and layers of Northern Italy (Adriatic Area 1 in Fig. 1). Contexts are presented in chronological-cultural order. GM: gnawing marks; TOT CM: total carnivore marks; D: digested.

\begin{tabular}{|c|c|c|c|c|c|c|}
\hline Sites & US/levels & GM & (D)igested & TOT.Car.M & TOT \% & TOT. NR \\
\hline RS-Rio Secco & $5+8$ & 53 & 2 & 55 & 1.3 & 4301 \\
\hline RF-Fumane & A9 & 100 & 1 & 101 & 0.09 & 111841 \\
\hline RF-Fumane & A6 & 24 & 16 & 40 & 0.03 & 111044 \\
\hline RF-Fumane & $\mathrm{A} 5 / \mathrm{A} 5+\mathrm{A} 6$ & 20 & 9 & 29 & 0.04 & 67083 \\
\hline RS-Rio Secco & 5 top +7 & 31 & - & 31 & 5.9 & 524 \\
\hline SB-S. Bernardino & $\mathrm{II}+\mathrm{III}$ & 61 & 1 & 62 & 0.6 & 9217 \\
\hline RF-Fumane & A4 & 51 & 17 & 68 & 0.3 & 19955 \\
\hline RB-Broion & $1 e+1 f+1 g$ & 3 & 1 & 4 & 0.01 & 37390 \\
\hline RF-Fumane & A3 & 53 & 36 & 89 & 0.5 & 16989 \\
\hline RF-Fumane & $\mathrm{A} 2-\mathrm{A} 2 \mathrm{R}$ & 17 & 9 & 26 & 0.1 & 19829 \\
\hline
\end{tabular}

\section{Table A.13}

Results of Mann-Whitney test for assessing significant differences in the distribution of fragment size classes and the proportion of burned and calcinated remains across Uluzzian and Late Mousterian layers of Northern Italy. The test was run on arcsine-transformed proportions.

\begin{tabular}{|c|c|c|}
\hline & Mann-Whitney W & P-value \\
\hline 1-3 cm LM - UL Northern Italy & 7 & 1 \\
\hline$>3 \mathrm{~cm} \mathrm{LM} \mathrm{-} \mathrm{UL} \mathrm{Northern} \mathrm{Italy}$ & 4 & 0.5 \\
\hline Burn. + Calc LM - UL Northern Italy & 5 & 0.86 \\
\hline
\end{tabular}

Table A.14

Results of test for differences in proportion of fragment size classes between Uluzzian and Late Mousterian layers of southeastern Italy (i.e. those with no direct evidence of carnivore gnawing) with relative effect size and statistical power.

\begin{tabular}{|c|c|c|c|c|c|}
\hline & $\mathrm{X}$-squared & $\mathrm{df}$ & P-value & Effect size (Cohen's h) & Power \\
\hline $1-3 \mathrm{~cm}$ CAV UL - CAV LM & 128.7 & 1 & $<0.001$ & -0.19 & 1 \\
\hline $1-3 \mathrm{~cm}$ CAV UL - OSC LM & 1875.8 & 1 & $<0.001$ & -0.55 & 1 \\
\hline$>3 \mathrm{~cm}$ CAV UL - CAV LM & 128.7 & 1 & $<0.001$ & 0.19 & 1 \\
\hline$>3 \mathrm{~cm} \mathrm{CAV} \mathrm{UL} \mathrm{-} \mathrm{OSC} \mathrm{LM}$ & 1875.8 & 1 & $<0.001$ & 0.58 & 1 \\
\hline Burn + Calc CAV UL - CAV LM & 4264.7 & 1 & $<0.001$ & 1.1 & 1 \\
\hline Burn + Calc CAV UL - OSC LM & 2.4161 & 1 & 0.12 & -0.04 & 0.72 \\
\hline
\end{tabular}

Table A.15

Results of test for differences in proportion of carpal/tarsal and phalanges/sesamoides of Bos primigenius between Uluzzian and Late Mousterian layers of southeastern Italy (i.e. those with no direct evidence of carnivore gnawing) with the relative effect size and statistical power.

\begin{tabular}{|c|c|c|c|c|c|}
\hline & $\mathrm{X}$-squared & $\mathrm{df}$ & P-value & Effect size (Cohen's h) & Power \\
\hline Carpal + tarsal CAV UL - CAV LM & 19.344 & 1 & $<0.001$ & 0.45 & 0.965 \\
\hline Carpal + tarsal CAV UL - OSC LM & 52.104 & 1 & $<0.001$ & 0.69 & 0.999 \\
\hline Phalang. + Sesamoides CAV UL - CAV LM & 59.942 & 1 & $<0.001$ & 0.81 & 0.999 \\
\hline Phalang. + Sesamoides CAV UL - OSC LM & 97.192 & 1 & $<0.001$ & 0.93 & 1 \\
\hline
\end{tabular}

Table A.16

Results of test for differences in proportion of carpal/tarsal and phalanges/sesamoides across all ungulates between Uluzzian and Late Mousterian layers of southeastern Italy (i.e. those with no direct evidence of carnivore gnawing) with the relative effect size and statistical power.

\begin{tabular}{|c|c|c|c|c|c|}
\hline & $\mathrm{X}$-squared & df & P-value & Effect size (Cohen's h) & Power \\
\hline Carpal + tarsal CAV UL - CAV LM & 79.232 & 1 & $<0.001$ & 0.57 & 0.999 \\
\hline Carpal + tarsal CAV UL - OSC LM & 20.831 & 1 & $<0.001$ & 0.55 & 0.999 \\
\hline Phalanges + Sesamoides CAV UL - CAV LM & 73.523 & 1 & $<0.001$ & 0.59 & 0.999 \\
\hline Phalanges + Sesamoides CAV UL - OSC LM & 51.12 & 1 & $<0.001$ & 0.83 & 1 \\
\hline
\end{tabular}


Table A.17

Results of test for differences in proportion of diaphysis, epiphysis, and spongy bones between Uluzzian and Late Mousterian layers of southeastern Italy (i.e. those with no direct evidence of carnivore gnawing) with the relative effect size and statistical power.

\begin{tabular}{|c|c|c|c|c|c|}
\hline & $\mathrm{X}$-squared & df & P-value & Effect size (Cohen's h) & Power \\
\hline Diaphysis CAV UL - CAV LM & 780.01 & 1 & $<0.001$ & 0.5 & 1 \\
\hline Diaphysis CAV UL - OSC LM & 883.87 & 1 & $<0.001$ & 0.58 & 1 \\
\hline Epiphysis CAV UL - CAV LM & 83.663 & 1 & $<0.001$ & 0.15 & 1 \\
\hline Epiphysis CAV UL - OSC LM & 176.26 & 1 & $<0.001$ & 0.25 & 1 \\
\hline Spongy bones CAV UL - CAV LM & 910.5 & 1 & $<0.001$ & 0.55 & 1 \\
\hline Spongy bones CAV UL - OSC LM & 142.45 & 1 & $<0.001$ & 0.23 & 1 \\
\hline
\end{tabular}

\section{References}

Alhaique, F., 2000. Analisi preliminare dei reperti faunistici associati al Paleolitico superiore del Riparo Mochi (Balzi Rossi, IM): scavi 1995-1996. Atti del $2^{\circ}$ Convegno Nazionale di Archeozoologia, Asti 1997, 125-130.

Alhaique, F., Tagliacozzo, A., 2000. L'interpretazione dei dati faunistici nella ricostruzione delle strategie di sussistenza nel Paleolitico medio: l'esempio del Lazio. In: Atti del $2^{\circ}$ Convegno Nazionale di Archeozoologia. ABACO Edizioni, Forlì, pp. $111-124$.

Allen, J.R.M., Brandt, U., Brauer, A., Hubbertens, A.W., Huntley, B., Keller, J., Kraml, M., Mackensen, A., Mingram, J., Negendank, J.F.W., Nowaczyk, N.R., Oberhänsli, H., Watts, W.A., Wulf, S., Zolitschka, B., 1999. Rapid environmental changes in southern Europe during the last glacial period. Nature 400, 740-743.

Benazzi, S., Douka, K., Fornai, C., Bauer, C.C., Kullmer, O., Svoboda, J., Pap, I., Mallegni, F., Bayle, P., Coquerelle, M., Condemi, S., Ronchitelli, A., Harvati, K., Weber, G.W., 2011. Early dispersal of modern humans in Europe and implications for Neanderthal behaviour. Nature 479, 525-528.

Benini, A., Boscato, P., Gambassini, P., 1997. Grotta della Cala (Salerno): industrie litiche e faune uluzziane ed aurignaziane. Riv. Sci. Preist 48, 37-96.

Benjamin, J., Rovere, A., Fontana, A., Furlani, S., Vacchi, M., Inglis, R.H.H., Galili, E., Antonioli, F., Sivan, D., Miko, S., Mourtzas, N., Felja, I., Meredith-Williams, M., Goodman-Tchernov, B., Kolaiti, E., Anzidei, M., Gehrels, R., 2017. Late Quaternary sea-level changes and early human societies in the central and eastern Mediterranean Basin: an interdisciplinary review. Quat. Int. 449, 29-57.

Bertola, S., Broglio, A., Cristiani, E., De Stefani, M., Gurioli, F., Negrino, F., Romandini, M., Vanhaeren, M., 2013. La diffusione del primo Aurignaziano a sud dell'arco alpino. Preistoria Alp. 47, 123-152.

Bevan, A., Conolly, J., Hennig, C., Johnston, A., Quercia, A., Spencer, L., Vroom, J., 2013. Measuring chronological uncertainty in intensive survey finds: a case study from Antikythera, Greece. Archaeom 55 (2), 312-328.

Bietti, A., Grimaldi, S. (Eds.), 1996. Reduction Processes (Chaînes Opératoires) in the European Mousterian. Quaternaria Nova 6.

Bietti, A., Manzi, G. (Eds.), 1990-91. The Fossil Man of Monte Circeo. Fifty Years of Studies on the Neanderthals in Latium. Quaternaria Nova 1.

Binford, L.R., 1981. Bones, Ancient Men and Modern Myths. Academic Press, New York. Binford, L.R., 1984. Faunal remains from Klasies River Mouth Academic Press, New York.

Blumenschine, R.J., 1995. Percussion marks, tooth marks, and experimental determinations of the timing of hominid and carnivore ace to long bones at FLK Zinjanthropus, Olduvai Gorge, Tanzania. J. Hum. Evol. 27, 197-213.

Blumenschine, R.J., Selvaggio, M.M., 1988. Percussion marks on bone surfaces as a new diagnostic of hominid behavior. Nature 333, 763-765.

Bond, G., Heinrich, H., Broecker, W., Labeyrie, L., McManus, J., Andrews, J., Huon, S. Jantschik, R., Clasen, S., Simet, C., Tedesco, K., Klas, M., Bonani, G., Ivy, S., 1992. Evidence for massive discharges of icebergs into North Atlantic Ocean during the last glacial period. Nature 360, 245-249.

Boscato, P., 2017. Ambienti ed economia nel Paleolitico medio della Puglia: lo studio delle faune. In: Radina, F. (Ed.), Preistoria e Protostoria della Puglia, Studi di Preistoria e Protostoria 4, pp. 119-124.

Boscato, P., Crezzini, J., 2006. The exploitation of ungulate bones in Homo neanderthalensis and Homo sapiens. Hum. Evol. 21 (3-4), 311-320.

Boscato, P., Crezzini, J., 2012. Middle-Upper Palaeolithic transition in Southern Italy: Uluzzian macromammals from Grotta del Cavallo (Apulia). Quat. Int. 252, 90-98.

Boscato, P., Ronchitelli, A., 2008. Strutture di combustione in depositi del Paleolitico medio del Sud Italia. Int. J. of Anthropol. 218-225 special issue.

Boscato, P., Ronchitelli, A., Wierer, U., 1997. Il Gravettiano antico della Grotta della Cala a Marina di Camerota (SA): paletnologia e ambiente. Riv. Sci. Preist 48, 97-186.

Boscato, P., Gambassini, P., Ronchitelli, A., 2004. Abri "L'Oscurusciuto" à Ginosa (Taranto - Italie du Sud): un nouveau site moustérien. In: Actes du XIVème Congrès UISPP, Université de Liege, Belgique, Sect. 5: Le Paléolithique Moyen. BAR International Series 1239, pp. 275-282

Boscato, P., Boschian, G., Caramia, F., Gambassini, P., 2009. Il Riparo del Poggio a Marina di Camerota (Salerno): culture e ambiente. Riv. Sci. Preist LIX, 5-40.

Boscato, P., Gambassini, P., Ranaldo, F., Ronchitelli, A., 2011. Management of paleoenvironmental resources and raw materials exploitation at the middle paleolithic site of Oscurusciuto (Ginosa, southern Italy): units 1 and 4. In: Conard, N.J., Richter, J. (Eds.), Neanderthal Lifeways, Subsistence and Technology - One Hundred Fifty Years of Neanderthal Study, pp. 87-98.

Brichetti, P., Fracasso, G., 2007. Ornitologia italiana. Vol. 4: Apodidae-Prunellidae.
Identificazione, distribuzione, consistenza e movimenti degli Uccelli italiani. Alberto Perdisa Editore, Bologna.

Brichetti, P., Fracasso, G., 2011. Ornitologia italiana. Vol. 7: Paridae-Corvidae. Identificazione, distribuzione, consistenza e movimenti degli Uccelli italiani. Alberto Perdisa Editore, Bologna.

Brichetti, P., Fracasso, G., 2015. Check-list degli uccelli italiani aggiornata al 2014. Riv. Ita. di Ornitol 85, 31-50.

Bronk Ramsey, C., 2009. Bayesian analysis of radiocarbon dates. Radiocarbon 51 (1), 337-360.

Capaldo, S.D., Blumenschine, R.J., 1994. A quantitative diagnosis of notches made by hammerstone percussion and carnivore gnawing on bovid long bones. Am. Antiq. 59, 724-748.

Carrera, L., Pavia, M., Peresani, M., Romandini, M., 2018a. Late Pleistocene fossil birds from Buso Doppio del Broion Cave (North-Eastern Italy): implications for palaeoecology, palaeoenvironment and palaeoclimate. Boll. Soc. Paleontol. Ita 57 (2), 145-174.

Carrera, L., Pavia, M., Romandini, M., Peresani, M., 2018b. Avian fossil assemblages at the onset of the LGM in the eastern Alps: a palaecological contribution from the Rio Secco cave (Italy). C.R. Palevol 17, 166-177.

Cassoli, P.F., Tagliacozzo, A., 1994. Considerazioni paleontologiche, paleoecologiche e archeozoologiche sui macromammiferi e gli uccelli dei livelli del Pleistocene superiore del Riparo di Fumane (VR) scavi 1988-91. Boll. Museo Civico di Storia Naturale di Verona 23, 85-117.

Cassoli, P.F., Tagliacozzo, A., 1994. I resti ossei di macromammiferi, uccelli e pesci della Grotta maggiore di San Bernardino sui Colli Berici (VI): considerazioni paleoeconomiche, paleoecologiche e cronologiche. Bull. di Paletnol. Ital 85, 1-71.

Cassoli, P.F., Tagliacozzo, A., 1997. Avifauna e Ittiofauna di Grotta di Castelcivita: considerazioni ecologiche ed inquadramento crono-stratigrafico. In: Gambassini, P. (Ed.), Il Paleolitico di Castelcivita, culture e ambiente. Materiae 5. Electa, Napoli, pp. $60-74$.

Castelletti, L., Maspero, A., 1997. Le analisi antracologiche della Grotta di Castelcivita. In: Gambassini, P. (Ed.), Il Paleolitico di Castelcivita: culture e ambiente. Electa, Napoli, pp. 75-91.

Cattani, L., Renault-Miskovsky, J., 1983-84. Etude pollinique du remplissage de la Grotte du Broion (Vicenza, Italie) : paléoclimatologie du Würmien en Vénétie. Bull. Assoc. Fr. Étude Quat. XVI (4), 197-212.

Champely, S., 2018. Pwr: Basic Funtions for Power Analysis. R package version 1.2-2. https://CRAN.R-project.org/package $=$ pwr.

Collard, M., Tarle, L., Sandgathe, D., Allan, A., 2016. Faunal evidence for a difference in clothing use between Neanderthals and early modern humans in Europe. J. Anthropol. Archaeol. 44, 235-246.

Costamagno, S., Rigaud, J.P., 2014. L'exploitation de la graisse au Paléolithique. In: Costamagno, S. (Ed.), Histoire de l'alimentation humaine: entre choix et contraintes,138ème Congrès du CTHS, Rennes, 22-27 Avril 2013. CTHS, Paris, pp. $134-152$.

Cramp, S., 1998. The Complete Birds of the Western Palearctic. Oxford University Press.

Crema, E.R., 2012. Modelling temporal uncertainty in archaeological analysis. J. Archaeol. Method Theory 19 (3), 440-461.

Dansgaard, W., Johnsen, S.J., Clausen, H.B., Dahl-Jensen, D., Gundestrup, N.S., Hammer, C.U., Hvidberg, C.S., Steffensen, J.P., Sveinbjörnsdottir, A.E., Jouzel, J., Bond, G., 1993. Evidence for general instability of past climate from 250-Kyr ice-core record. Nature 364, 218-220.

De Stefani, M., Gurioli, F., Ziggiotti, S., 2005. Il Paleolitico superiore del Riparo del Broion nei Colli Berici (Vicenza). Riv. Sci. Preist. Suppl 1, 93-107.

Douka, K., Grimaldi, S., Boschian, G., Del Lucchese, A., Higham, T.F.G., 2012. A new chronostratigraphic frame work for the Upper Palaeolithic of Riparo Mochi (Italy). J. Hum. Evol. 62, 286-299.

Douka, K., Higham, T.F., Wood, R., Boscato, P., Gambassini, P., Karkanas, P., Peresani, M., Ronchitelli, A.M., 2014. On the chronology of the Uluzzian. J. Hum. Evol. 68, $1-13$.

Duches, R., Nannini, N., Romandini, M., Boschin, F., Crezzini, J., Peresani, M., 2016. Identification of Late Epigravettian hunting injuries: descriptive and 3D analysis of experimental projectile impact marks on bone. J. Archaeol. Sci. 66, 88-102.

Emerson, A.M., 1990. Archaeological Implications of Variability in the Economic Anatomy of Bison bison. Unpublished Ph. D. Dissertation. Washington State University, Pullman.

Emerson, A.M., 1993. The role of body Part Utility in small-scale hunting under two strategies of carcass recovery. In: Hudson, J. (Ed.), From Bones to Behavior: Ethnoarchaeology and Experimental Contributions to the Interpretation of Faunal 
Remains. Southern Illinois University at Carbondale Center for Archaeological Investigations, Occasional Paper 2, Carbondale, pp. 138-155.

Fabbri, P.F., Panetta, D., Sart, L., Martini, F., Salvadori, P.A., Caramelli, D., Fedi, M., Benazzi, S., 2016. Middle Palaeolithic human deciduous incisor from Grotta del Cavallo. Italy. Am. J. Phys. Anthropol. 161 (3), 506-512. https://doi.org/10.1002/ ajpa.23044.

Fernández-Jalvo, Y., Andrews, P., 2016. Atlas of Taphonomic Identifications. Vertebrate Paleobiology and Paleoanthropology. Springer, Dordrecht.

Fiore, I., Gala, M., Tagliacozzo, A., 2004. Ecology and subsistence strategies in the eastern Italian Alps during the middle palaeolithic. Int. J. Osteoarchaeol. 14 (3-4), 273-286.

Fiore, I., Gala, M., Boschin, F., Crezzini, J., Tagliacozzo, A., Moroni, A., 2019. Archeozoology and taphonomy of bird remains from Grotta di Castelcivita (Salerno, Italy) and clues for human-bird interactions. Quat. Int. https://doi.org/10.1016/j. quaint.2019.09.004.

Fiore, I., Gala, M., Romandini, M., Cocca, E., Tagliacozzo, A., Peresani, M., 2016. From feathers to food: reconstructing the complete exploitation of avifaunal resources by Neanderthals at Grotta di Fumane, unit A9. Quat. Int. 421, 134-153.

Fisher, W.J., 1995. Bone surface modifications in zooarchaeology. J. Archaeol. Method Theory 2, 7-68.

Fletcher, W.J., Goni, M.F.S., Allen, J.R., Cheddadi, R., Combourieu-Nebout, N., Huntley, B., Lawson, I., Londeix, L., Magri, D., Margari, V., Müller, U., Naughton, F., Novenko, E., Roucoux, K., Tzedakis, P.C., 2010. Millennial-scale variability during the last glacial in vegetation records from Europe. Quat. Sci. Rev. 29 (21-22), 2839-2864.

Gala, M., Tagliacozzo, A., 2005. L'avifauna dei livelli aurignaziani di Grotta di Fumane (VR). Risultati preliminari dello studio tafonomico. In: Malerba, G., Visentini, P. (Eds.), Atti del $4^{\circ}$ Convegno Nazionale di Archeozoologia. Pordenone 13-15 novembre 2003. Quaderni del Museo Archeologico del Friuli Occidentale 6, Pordenone, pp. 53-57.

Gala, M., Tagliacozzo, A., 2010. The avifauna from Late Glacial archaeological sites in Italy: a tentative synthesis. In: Prummel, W., Zeiler, J.T., Brinkhuizen, D.C. (Eds.), Birds in Archaeology. Proceedings of the 6th Meeting of the ICAZ Bird Working Group in Groningen, Gronigen Archaeological Studies 12, Barkhuis, pp. 205-218.

Gala, M., Fiore, I., Tagliacozzo, A., 2018. Human exploitation of avifauna during the Italian middle and upper paleolithic. In: Borgia, V., Cristiani, E. (Eds.), Palaeolithic Italy: Advanced Studies on Early Human Adaptations in the Apennine Peninsula. Sidestone Press, Leiden, pp. 183-217.

Gambassini, P. (Ed.), 1997. Il Paleolitico di Castelcivita: culture e ambiente. Electa, Napoli.

Geneviève Pothier Bouchard, Julien Riel-Salvatore, Fabio Negrino, Michael Buckley (in press) Archaeozoological, Taphonomic and ZooMS Insights into the Protoaurignacian Faunal Record from Riparo Bombrini, Quaternary International.

Giaccio, B., Hajdas, I., Isaia, R., Deino, A., Nomade, S., 2017. High-precision 14C and $40 \mathrm{Ar} / 39 \mathrm{Ar}$ dating of the Campanian Ignimbrite (Y-5) reconciles the time-scales of climatic-cultural processes at 40 ka. Sci. Rep. 7, 45940. https://doi.org/10.1038/ srep45940.

Grayson, D.K., 1984. Quantitative Zooarchaeology: Topics in the Analysis of Archaeological Faunas. Academic Press, Orlando.

Grimaldi, S., Porraz, G., Santaniello, F., 2014. Raw material procurement and land use in the northern mediterranean arc: insight from the first proto-aurignacian of Riparo Mochi (Balzi Rossi, Italy). Quartar 61, 113-127.

Gruppioni, G., 2003. Datation par les méthodes Uranium-Thorium (U/Th) et Resonance Paramagnetique Electronique (RPE) de deux gisements du Paléolithique moyen et supérieur de Vénetie: la Grotta de Fumane (Monts Lessini - Verone) et la Grotte Majeure de San Bernardino (Monts Berici - Vicence) (PhD Dissertation). University of Ferrara.

Guidi, A., Piperno, M., 1992. Italia Preistorica. Laterza).

Gurioli, F., Cappato, N., De Stefani, M., Tagliacozzo, A., 2006. Considerazioni Paleontologiche, Paleoecologiche e Archeozoologiche dei livelli del Paleolitico superiore del Riparo del Broion (Colli Berici, Vicenza). In: Tagliacozzo, A., Fiore, I., Marconi, S., Tecchiati, U. (Eds.), Atti del $5^{\circ}$ Convegno Nazionale di Archeozoologia, Rovereto, Italy, pp. 47-56.

Higham, T., 2011. European Middle and Upper Palaeolithic radiocarbon dates are often older than they look: problems with previous dates and some remedies. Antiquity 85 (327), 235-249.

Higham, T., Brock, F., Peresani, M., Broglio, A., Wood, R., Douka, K., 2009. Problems with radiocarbon dating the middle and upper palaeolithic transition in Italy. Quat. Sci. Rev. 28, 1257-1267.

Higham, T., Compton, T., Stringer, C., Jacobi, R., Shapiro, B., Trinkaus, E., Chandler, B., Gröning, F., Collins, C., Hillson, S., O'Higgins, T., FitzGerald, C., Fagan, M., 2011. The earliest evidence for anatomically modern humans in northwestern Europe. Nature 479, 521-524.

Higham, T., Douka, K., Wood, R., et al., 2014. The timing and spatiotemporal patterning of Neanderthal disappearance. Nature 512, 306-309. https://doi.org/10.1038/ nature13621.

Hockett, B., Haws, J.A., 2005. Nutritional ecology and the human demography of Neandertal extinction. Quat. Int. 137, 21-34.

Holt, B., Negrino, F., Riel-Salvatore, J., Formicola, V., Arellano, A., Arobba, D., Boschian, G., Churchill, S.E., Cristiani, E., Canzio, E., Di Vicino, G., 2019. The middle-upper paleolithic transition in northwest Italy: new evidence from Riparo Bombrini (Balzi Rossi, Liguria, Italy). Quat. Int. 508, 142-152.

Ivana Fiore, MonicaGala, Francesco Boschin, Jacopo Crezzini, Antonio Tagliacozzo, Adriana Moroni, (corrected proof, in press) Archeozoology and taphonomy of bird remains from Grotta di Castelcivita (Salerno, Italy) and clues for human-bird interactions, Quaternary International.

Jéquier, C., Peresani, M., Livraghi, A., Romandini, M., 2018. Same but different: 20,000 years of bone retouchers from Northern Italy. A diachronologic approach from
Neanderthals to anatomically modern humans. In: Hutson, J.M., García-Moreno, A., Noack, E., Turner, E., Villaluenga, A., Gaudzinski-Windheuser, S. (Eds.), The Origins of Bone Tool Technologies. Römisch-Germanisches Zentralmuseum - TAGUNGEN. 35. Verlag des Römisch-Germanischen Zentralmuseums, Band, pp. 269-285.

Johnson, I., 2004. Aoristic analysis: seeds of a new approach to mapping archaeological distributions through time. In: Ausserer, K.F., Börner, W., Goriany, M., KarlhuberVöckl, L. (Eds.), [Enter the Past] the E-Way into the Four Dimensions of Cultural Heritage: CAA2003. Archaeopress, Oxford, pp. 448-452.

Klein, R.G., Scott, K., 1986. Re-analysis of faunal assemblages from the Haua Fteah and other late Quaternary archaeological sites in Cyrenaican Libya. J. Archaeol. Sci. 13, 515-542 Academic Press.

Kowalewski, M., 1996. Time-averaging, overcompleteness, and the geological record. J. Geol. 104, 317-326.

Kuhn, S.L., Bietti, A., 2000. The late middle and early upper paleolithic in Italy. In: BarYosef, O., Pilbeam, D. (Eds.), The Geography of Neandertals and Modern Humans in Europe and the Greater Mediterranean. Peabody Museum of Archaeology and Ethnology, Cambridge, MA, pp. 49-72.

Kuhn, S.L., Stiner, M.C., 1998. The earliest aurignacian of Riparo Mochi (Liguria, Italy. Curr. Anthropol. 39 (Suppl. 3), 175-188.

Leonard, R.D., Jones, G.T. (Eds.), 1989. Quantifying Diversity in Archaeology. Cambridge University Press, Cambridge.

Leonardi, P., Broglio, A., 1966. Datazione assoluta di un'industria musteriana della Grotta del Broion. Riv. Sci. Preist 21 (2), 397-405.

López-García, J.M., dalla Valle, C., Cremaschi, M., Peresani, M., 2015. Reconstruction of the Neanderthal and Modern Human landscape and climate from the Fumane cave sequence (Verona, Italy) using small-mammal assemblages. Quat. Sci. Rev. 128, $1-13$.

López-García, J.M., Luzi, E., Peresani, M., 2017. Middle to Late Pleistocene environmental and climatic reconstruction of the human occurrence at Grotta Maggiore di San Bernardino (Vicenza, Italy) through the small-mammal assemblage. Quat. Sci. Rev. 168, 42-54.

Lyman, R.L., 1994. Vertebrate Taphonomy. Cambridge University Press, Cambridge.

Lyman, R.L., 2008. Quantitative Paleozoology. Cambridge University Presshttps://doi. org/10.1017/CBO9780511813863.

Madsen, M.E., 2018. Neutral cultural transmission in time averaged archaeological assemblages. https://arxiv.org/abs/1204.2043.

Marciani, G., Spagnolo, V., Aureli, D., Ranaldo, F., Boscato, P., Ronchitelli, A., 2016. Middle Palaeolithic technical behaviour: material import-export and Levallois production at the SU 13 of Oscurusciuto rock shelter, Southern Italy. J. Lit. Stud. 3 (2), $1-24$.

Marciani, G., Arrighi, S., Aureli, D., Spagnolo, V., Boscato, P., Ronchitelli, A., 2018. Middle Palaeolithic lithic tools. Techno-functional and use-wear analysis of target objects from us 13 at the Oscurusciuto rock shelter, southern Italy. J. Lit. Stud. 5 (2), $1-30$.

Margari, V., Gibbard, P.L., Bryant, C.L., Tzedakis, P.C., 2009. Character of vegetational and environmental changes in southern Europe during the last glacial period; evidence from Lesvos Island, Greece. Quat. Sci. Rev. 28 (13-14), 1317-1339.

Martini, I., Ronchitelli, A., Arrighi, S., Capecchi, G., Ricci, S., Scaramucci, S., Spagnolo, V., Gambassini, P., Moroni, A., 2018. Cave clastic sediments as a tool for refining the study of human occupation of prehistoric sites: insights from the cave site of La Cala (Cilento, southern Italy). J. Quat. Sci. 33, 586-596.

Masini, F., Abbazzi, L., 1997. L'associazione di mammiferi della Grotta di Castelcivita. In: Gambassini, P. (Ed.), Il Paleolitico di Castelcivita, culture e ambiente. Materiae 5. Electa, Napoli, pp. 33-59.

Masini, F., Sala, B., 2007. Stratigraphic distribution patterns of large and small mammals in the late Pliocene and Pleistocene of the Italian peninsula: an integrated approach. Quat. Int. 160 (1), 43-56.

Masini, F., Sala, B., 2011. Considerations on an integrated biochronological scale of Italian quaternary continental mammals. Il Quaternario. Ital. J. Quat. Sci. 24 (2), 193-198.

Milliken, S., 1999-2000. The Neanderthals in Italy. Accordia Research Papers 8, 1-82.

Morin, E., 2006. Fat composition and Nunamiut decision-making: a new look at the marrow and bone grease indices J. Arch. Sci. 20, 1-14.

Morin, E., Ready, E., Boileau, A., Beauval, C., Coumont, M.P., 2017. Problems of identification and quantificationin archaeozoological analysis, Part I: insights from a blind test. J. Archaeol. Method Theory 24, 886-937.

Morin, E., Meier, J., El Guennouni, K., Moigne, A.M., Lebreton, L., Rusch, L., Valensi, P., Conolloy, J., Cochard, D., 2019. New evidence of broader diets for archaic Homo populations in the northwestern Mediterranean. Sci. Adv 5, 1-12.

Moroni, A., Boscato, P., Ronchitelli, A., 2013. What roots for the Uluzzian? Modern behaviour in Central-Southern Italy and hypotheses on AMH dispersal routes. Quat. Int. $316,27-44$.

Moroni, A., Ronchitelli, A., Arrighi, S., Aureli, D., Bailey, S.E., Boscato, P., Boschin, F. Capecchi, G., Crezzini, J., Douka, K., Marciani, G., Panetta, D., Ranaldo, F., Ricci, S., Scaramucci, S., Spagnolo, V., Benazzi, S., Gambassini, P., 2018. Grotta del Cavallo (Apulia - southern Italy). The Uluzzian in the mirror. J. Anthropol. Sci. 96, 1-36.

Moroni, A., Boschian, G., Crezzini, J., Montanari-Canini, G., Marciani, G., Capecchi, G., Arrighi, S., Aureli, D., Berto, C., Freguglia, M., Araujo, A., Scaramucci, S., Hublin, J.J., Lauer, T., Benazzi, S., Parenti, F., Bonato, M., Ricci, S., Talamo, S., Segre, A.G., Boschin, F., Spagnolo, V., 2019. Late Neandertals in central Italy. High-resolution chronicles from Grotta dei Santi (Monte Argentario-Tuscany). Quat. Sci. Rev. 217, 130-151. https://doi.org/10.1016/j.quascirev.2018.11.021.

Müller, U.C., Pross, J., Tzedakis, P.C., Gamble, C., Kotthoff, U., Schmiedl, G., Wulf, S., Christanis, K., 2011. The role of climate in the spread of modern humans into Europe. Quat. Sci. Rev. 30, 273-279.

Mussi, M., 2001. Earliest Italy. An Overview of the Italian Paleolithic and Mesolithic. 
Kluwer Academic/Plenum Publishers

Negrino, F., Riel-Salvatore, J., 2018. From Neanderthals to anatomically modern humans in Liguria (Italy): the current state of knowledge. In: Borgia, V., Cristiani, E. (Eds.), Out of Italy, Advanced Studies on Early Human Adaptations in the Apennine Peninsula. Sidestone Press Academics, Leida, pp. 159-180.

Onoratini, G., 2004. Le Protoaurignacien, première culture de l'Homme Moderne de Provence et Ligurie. L'Anthropol 108, 239-249.

Onoratini, G., Simon, P., 2006. Le Protoaurignacien de la Grotte de l'Observatoire (Principauté de Monaco). In: Sanchidrian Torti, J.L., Marquez Alcantara, A.M., Fullola i Pericot, J.M. (Eds.), La Cuenca Mediterranea durante el Paleolitico Superior, IV Simposio de Prehistoria Cueva de Nerja, pp. 430-443.

Orton, D., 2017. archSeries: tools for chronological uncertainty in archaeology. R package version 0.0.0.9003. https://github.com/davidcorton/archSeries.

Orton, D., Morris, J., Pipe, A., 2017. Catch per unit research effort: sampling intensity, chronological uncertainty, and the onset of marine fish consumption in historic London. Open Quat. 3 (1), 1-20.

Palma di Cesnola, A., 1993. Il Paleolitico Superiore in Italia. Garlatti e Razzai Ed, Firenze.

Pavia, M., 1999. The middle Pleistocene avifauna of spinagallo cave (sicily, Italy): preliminary report. Smithson. Contrib. Paleobiol. 89, 125-127.

Peresani, M., 2001. An overview of the Middle Palaeolithic settlement system in NorthEastern Italy. In: Conard, N.J. (Ed.), Settlement Dynamics of the Middle Palaeolithic and Middle Stone Age. Tübingen Publications in Prehistory, Introductory Volume, pp. 485-506.

Peresani, M., 2009. Neanderthal behaviour viewed across the isotopic Stage 3 in the alpine fringe of Italy. Gortania. Geologia, Paleontologia, Paletnologia 31, 87-96.

Peresani, M., 2011. The end of the Middle Palaeolithic in the Italian Alps. An overview on Neandertal land-use, subsistence and technolog. In: Conard, N., Richter, J. (Eds.), Neanderthal Lifeways, Subsistence and Technology. One Hundred Fifty Years of Neanderthal Study, Vertebrate Paleobiology and Paleoanthropology Series. Springer, New York, pp. 249-259.

Peresani, M., Cremaschi, M., Ferraro, F., Falgueres, Ch, Bahain, J.J., Gruppioni, G., Sibilia, E., Quarta, G., Calcagnile, L., Dolo, J.M., 2008. Age of the final middle palaeolithic and uluzzian levels at Fumane cave, northern Italy, using 14C, ESR, 234U/ 230Th and thermoluminescence methods. J. Archaeol. Sci. 35, 2986-2996.

Peresani, M., Fiore, I., Gala, M., Romandini, M., Tagliacozzo, A., 2011a. Late Neandertals and the intentional removal of feathers as evidenced from bird bone taphonomy at Fumane cave 44 Kyr BP, Italy. PNAS 108 (10), 3888-3893.

Peresani, M., Boldrin, M., Pasetti, P., 2015. Assessing the exploitation of double patinated artifacts from the Late Mousterian: Implications for lithic economy and human mo bility in northern Italy. Quat. Int. 361, 238-250.

Peresani, M., Chrzavzez, J., Danti, A., De March, M., Duches, R., Gurioli, F., Muratori, S., Romandini, M., Trombino, L., Tagliacozzo, A., 2011b. Fire-places, frequentations and environmental setting of the final Mousterian at Grotta di Fumane: a report from the 2006-2008 research. Quartar 58, 131-151.

Peresani, M., Romandini, M., Duches, R., Jéquier, C., Nannini, N., Pastors, A., Picin, A., Schmidt, I., Vaquero, M., Weniger, G.C., 2014. New evidence for the Neanderthal demise and earliest gravettian occurrences at Rio Secco cave, Italy. J. Field Archaeol. 39, 401-416.

Peresani, M., Cristiani, E., Romandini, M., 2016. The Uluzzian technology of Grotta di Fumane and its implication for reconstructing cultural dynamics in the Middle-Upper Palaeolithic transition of Western Eurasia. J. Hum. Evol. 9, 36-56. https://doi.org/ 10.1016/j.jhevol.2015.10.012.

Peresani, M., Bertola, S., Delpiano, D., Benazzi, S., Romandini, M., 2019. The Uluzzian in the north of Italy: insights around the new evidence at Riparo Broion. Archaeol. Anthropol. Sci 11 (7), 3503-3536. https://doi.org/10.1007/s12520-018-0770-z.

Pini, R., Ravazzi, C., Donegana, M., 2009. Pollen stratigraphy, vegetation and climate history of the last $215 \mathrm{Kyr}$ in the Azzano Decimo core (Plain of Friuly, northeastern Italy). Quat. Sci. Rev. 28, 1268e1290.

Pini, R., Ravazzi, C., Reimer, P.J., 2010. The vegetation and climate history of the last glacial cycle in a new pollen record from Lake Fimon (southern Alpine foreland, NItaly). Quat. Sci. Rev. 29, 3115e3137.

Pitti, C., Sorrentino, C., Tozzi, C., 1976. L'industria di tipo Paleolitico superiore arcaico della Grotta La Fabbrica (Grosseto). Nota preliminare. Atti Soc. Tosc. Sci. Nat., Mem., Serie A 83, 174-201.

Porraz, G., Simon, P., Pasquini, A., 2010. Identité technique et comportement économiques des groupes proto-aurignaciens à la Grotte de l'Observatoire (Principauté de Monaco). Gall. Prehist. 52, 33-59.

Pothier Bouchard G., Susan M. Mentzer M. S., Julien Riel-Salvatore J., Hodgkins J., Miller E. C., Negrino F., Wogelius R., Buckley M., 2019. Portable FTIR for on-site screening of archaeological bone intended for ZooMS collagen fingerprint analysis. J. Archaeol. Sci.: For. Rep. 26, 101-862.

Potts, R., Shipman, P., 1981. Cutmarks made by stone tools on bones from Olduvai Gorge, Tanzania. Nature 291, 577-580.

Premo, L.S., 2014. Cultural transmission and diversity in time-averaged assemblages. Curr. Anthropol. 55 (1), 105-114.

Rasmussen, S.O., Bigler, M., Blockley, S.P., Blunier, T., Buchardt, S.L., Clausen, H.B., Cvijanovic, I., Dahl-Jensen, D., Johnsen, S.J., Fischer, H., 2014. A stratigraphic framework for abrupt climatic changes during the Last Glacial period based on three synchronized Greenland ice-core records: refining and extending the INTIMATE event stratigraphy. Quat. Sci. Rev. 106, 14-28.

Ratcliffe, J.H., 2000. Aoristic analysis: the spatial interpretation of unspecific temporal events. Int. J. Geographical Inf. Sci 14 (7), 669-679.

Riel-Salvatore, J., Negrino, F., 2018a. Human adaptations to climatic change in Liguria across the middle-upper paleolithic transition. J. Quat. Sci. 33, 313-322.

Riel-Salvatore, J., Negrino, F., 2018b. Lithic technology, mobility and human niche construction in Early Upper Paleolithic Italy. In: Robinson, E., Sellet, F. (Eds.), Lithic
Technological Organization and Paleoenvironmental Change. Springer, NY, pp. $163-187$.

Riel-Salvatore, J., Ludeke, I.C., Negrino, F., Holt, M., BM, 2013. A spatial analysis of the late mousterian levels of Riparo Bombrini (Balzi Rossi, Italy). Can. J. Archaeol. 37 (1), 70-92.

Romandini, M., 2012. Analisi archeozoologica, tafonomica, paleontologica e spaziale dei livelli Uluzziani e tardo-Musteriani della Grotta di Fumane (VR). Variazioni e continuità strategico comportamentali umane in Italia Nord-Orientale: $i$ casi di Grotta del Col della Stria (VI) e Grotta del Rio Secco (PN). Ph.D. thesis. University of Ferrara, Italy.

Romandini, M., 2017. La Grotte dell'Observatoire (Monaco). Industrie sur matières dures animales, objecte de parure et observations archéozoologiques. Bull. Musée Anthropol. Préhist. de Monaco 57, 75-96.

Romandini, M., Gurioli, F., Parere, V., 2012. Oggetti ornamentali del Paleolitico superiore nei Colli Berici (VI). In: Atti del $6^{\circ}$ Convegno Nazionale Italiano di Archeozoologia. San Romano in Garfagnana, Lucca, Orecchiella (2009), pp. 113-116.

Romandini, M., Nannini, N., Tagliacozzo, A., Peresani, M., 2014a. The ungulate assemblage from layer A9 at Grotta di Fumane, Italy: a zooarchaeological contribution to the reconstruction of Neanderthal ecology. Quat. Int. 337, 11-27.

Romandini, M., Peresani, M., Laroulandie, V., Metz, L., Pastoors, A., Vaquero, M., Slimak, L., 2014b. Convergent evidence of eagle talons used by Late Neanderthals in Europe: a further assessment on symbolism. PLoS One 9 (7), e101278.

Romandini, M., Tagliacozzo, A., Fiore, I., Gala, M., Peresani, M., 2016a. Strategie di sfruttamento delle risorse animali dei livelli uluzziani di Grotta di Fumane (Verona). Sezione di Museologia Scientifica e Naturalistica 12 (1), 43-52.

Romandini, M., Fiore, I., Gala, M., Cestari, M., Guida, G., Tagliacozzo, A., Peresani, M., 2016b. Neanderthal scraping and manual handling of raptors wing bones: evidence from Fumane Cave. Experimental activities and comparison. Quat. Int. 421, 154-172.

Romandini, M., Terlato, G., Nannini, N., Tagliacozzo, A., Benazzi, S., Peresani, M., 2018a. Humans and Bears a Neanderthal tale. Reconstructing uncommon behaviors from zooarchaeological evidence in southern Europe. J. Archaeol. Sci. 90, 71-91.

Romandini, M., Thun Hohenstein, U., Fiore, I., Tagliacozzo, A., Perez, A., Lubrano, V. Terlato, G., Peresani, M., 2018b. Late Neandertals and the exploitation of small mammals in Northern Italy: fortuity, necessity or hunting variability? Quaternaire 29 (1), 61-67.

Rossoni-Notter, E., Notter, O., Simone, O.S., Simon, P., 2016. Acheulean in Monaco: observatoire cave and its singular occupations. Quat. Int. 411, 212-235.

Sala, B., 1990. Loess fauna in deposits of shelters and caves in the Veneto region and examples in other region of Italy. In: Cremaschi, M. (Ed.), The Loess in Northern and Central Italy: a Loess Basin between the Alps and the Mediterranean Region, Quaderni di Geodinamica Alpina e Quaternaria 1, pp. 139-149.

Sala, B., Marchetti, M., 2006. The Po Valley floodplain (Northern Italy): a transitional area between two zoogeographical areas during the Late Neogene and Quaternary. Courier Forsch.-Inst. Senckenberg 256, 321-328.

Sala, B., Masini, F., 2007. The late Pliocene and Pleistocene small mammal chronology in the Italian peninsula. Quat. Int. 160 (1), 4-16.

Sala, B., Thun Hohenstein, U., Bertolini, M., 2012. I macromammiferi. In: Peretto, C. (Ed.), L'insediamento musteriano di Grotta Reali (Rocchetta a Volturno, Molise, Italia). Annali dell'Università di Ferrara, Museologia Scientifica e Naturalistica 8/2: 25-26.

Sánchez Goñi, M.F., Turon, J.L., Eynaud, F., Gendreau, S., 2000. European climatic response to millennial-scale changes in the atmosphere-ocean system during the last glacial period. Quat. Res. 54, 394-403.

Sarti, L., Boscato, P., Lo Monaco, M., 2000. Il Musteriano finale di Grotta del Cavallo nel Salento: studio preliminare. Origini 22, 45-109.

Sarti, L., Boscato, P., Martini, F., Spagnoletti, A.P., 2002. Il Musteriano di Grotta del Cavallo, strati H e I: studio preliminare. Riv. Sci. Preist 52, 21-109.

Shipman, P., Rose, J., 1984. Cutmark mimics on modern fossil bovid bones. Curr. Anthropol. 25, 116-177.

Spagnolo, V., Marciani, G., Aureli, D., Berna, F., Boscato, P., Ranaldo, F., Ronchitelli, A., 2016. Between hearths and volcanic ash: the SU 13 palimpsest of the Oscurusciuto rock shelter (Ginosa - southern Italy): analytical and interpretative questions. Quat. Int. 417, 105-121.

Spagnolo, V., Marciani, G., Aureli, D., Berna, F., Toniello, G., Astudillo, F.A., Boscato, P., Boschin, F., Ronchitelli, A., 2018. Neanderthal activity and resting areas from stratigraphic unit 13 at the middle palaeolithic site of Oscurusciuto (Ginosa - Taranto, southern Italy). Quat. Sci. Rev. https://doi.org/10.1016/j.quascirev.2018.06.024.

Starkovich, B.M., 2012. Intensification of small game resources at Klissoura cave 1 (peloponnese, Greece) from the middle paleolithic to mesolithic. Quat. Int. 264, 17-31.

Starkovich, B.M., 2017. Paleolithic subsistence strategies and changes in site use at Klissoura Cave 1 (Peloponnese, Greece). J. Hum. Evol. 111, 63-84.

Starkovich, B.M., Ntinou, M., 2017. Climate change, human population growth, or both? Upper Paleolithic subsistence shifts in southern Greece. Quat. Int. 428, 17-32. https://doi.org/10.1016/j.quaint.2015.03.044.

Starkovich, B.M., Munro, N.D., Stiner, M.C., 2018. Terminal Pleistocene subsistence strategies and aquatic resource use in southern Greece. Quat. Int. 465, 162-176. https://doi.org/10.1016/j.quaint.2017.11.015.

Stiner, M.C., 1994. Honor Among Thieves. A Zooarchaeology Study of Neanderta Ecology. Princeton University Press, Princeton, NJ.

Stiner, M.C., 2001. Thirty years on the "broad spectrum revolution" and paleolithic demography. Proc. Natl. Acad. Sci. 98, 6993-6996.

Stiner, M.C., 2005. The Faunas of Hayonim Cave, Israel: a 200,000-Year Record of Paleolithic Diet, Demography and Society. American School of Prehistoric Research Bulletin 48. Peabody Museum of Archaeology and Ethnology. Harvard University, Cambridge.

Stiner, Munro, 2002. Approaches to Prehistoric Diet Breadth, Demography, and Prey 
Ranking Systems in Time and Space. J. Archaeol. Method Theory 9 (2), 181-214. https://doi.org/10.1023/A:1016530308865.

Stiner, M.C., Munro, N.D., 2011. On the evolution of diet and landscape during the upper paleolithic through mesolithic at franchthi cave (peloponnese, Greece). J. Hum. Evol. 60, 618-636.

Stiner, M.C., Kuhn, S.L., Weiner, S., Bar-Yosef, O., 1995. Differential burning, recrystallization and fragmentation of archaeological bone. J. Archaeol. Sci. 22, 223-237.

Tagliacozzo, A., Romandini, M., Fiore, I., Gala, M., Peresani, M., 2013. Animal exploitation strategies during the uluzzian at Grotta Fumane (verona, Italy). In: Clark, J.L., Speth, J.D. (Eds.), Zooarchaeology and Modern Human Origins: Human Hunting Behavior during the Later Pleistocene. Vertebrate Paleobiology and Paleoanthropology Series. Springer, Dordrecht, pp. 129-150.

Talamo, S., Peresani, M., Romandini, M., Duches, R., Jéquier, C., Nannini, N., Pastors, A., Picin, A., Vaquero, M., Weninger, G.C., Hublin, J.J., 2014. Detecting human presence at the border of the northeastern Italian Pre-Alps. 14C dating at Rio Secco Cave as expression of the first Gravettian and the late Mousterian in the northern Adriatic region. PLoS One 9 (4), e95376.

Terlato, G., Livraghi, A., Romandini, M., Peresani, M., 2019. Large bovids on Neanderthal menu: exploitation of Bison priscus and Bos primigenius in northeastern Italy. J. Archaeol. Sci.: For. Rep. 25, 129-143.

Tyrberg, T., 1991. Arctic, montane and steppe birds as glacial relicts in West Palearctic. Ornithol. Verh. 25, 29-49.

Tzedakis, P.C., Lawson, I.T., Frogley, M.R., Hewitt, G.M., Preece, R.C., 2002. Buffered tree population changes in a Quaternary refugium: evolutionary implications. Science 297
(5589), 2044-2047.

Valensi, P., Psathi, E., 2004. Faunal exploitation during the Middle Palaeolithic in southeastern France and north-western Italy. Int. J. Osteoarchaeol. 14, 256-272.

Van Andel, T.H., Davies, W. (Eds.), 2003. Neanderthals and Modern Humans in the European Landscape during the Last Glaciation: Archaeological Results of the Stage 3 Project. McDonald Institute for Archaeological Research monographs, Cambridge, UK.

Villa, P., Pollarolo, L., Conforti, J., Marra, F., Biagioni, C., Degano, I., Lucejko, J.J., Tozzi, C., Pennacchioni, M., Zanchetta, G., Nicosia, C., Martini, M., Sibilia, E., Panzeri, L., 2018. From Neandertals to modern humans: new data on the Uluzzian. PLoS One 13 (5) e0196786.

Wood, R.E., Douka, K., Boscato, P., Haesaerts, P., Sinitsyn, A., Higham, T.F.G., 2012. Testing the ABOx-SC method: dating known age charcoals associated with the Campanian Ignimbrite. Quat. Geochronol. 9, 16-26.

Wulf, S., Hardiman, M., Staff, R.A., Koutsodendris, A., Appelt, O., Blockley, S.P.E., Lowe, J.J., Manning, C.J., Ottolini, L., Schmitt, A.K., Smith, V.C., Tomlinson, E.L., Vakhrameeva, P., Knipping, M., Kotthoff, U., Milner, A.M., Müller, U.C., Christanis, K., Kalaitzidis, S., Tzedakis, C., Schmiedl, G., Pross, J., 2018. The marine isotope stage $1-5$ cryptotephra record of Tenaghi Philippon, Greece: towards a detailed tephrostratigraphic framework for the Eastern Mediterranean region. Quat. Sci. Rev. 186, 236-262.

Zanchetta, G., Giaccio, B., Bini, M., Sarti, L., 2018. Tephrostratigraphy of Grotta del Cavallo, Southern Italy: insights on the chronology of Middle to Upper Palaeolithic transition in the Mediterranean. Quat. Sci. Rev. 182, 65-77. 